Article

\title{
Orthogonal Gyrodecompositions of Real Inner Product Gyrogroups
}

\author{
Milton Ferreira ${ }^{1, *,+}+\left(\right.$ ) and Teerapong Suksumran ${ }^{2}$ (I) \\ 1 School of Technology and Management, Polytechnic Institute of Leiria, 2411-901 Leiria, Portugal \\ 2 Research Center in Mathematics and Applied Mathematics, Department of Mathematics, Faculty of Science, \\ Chiang Mai University, Chiang Mai 50200, Thailand; teerapong.suksumran@cmu.ac.th \\ * Correspondence: milton.ferreira@ipleiria.pt \\ + Research Center: CIDMA-Center for Research and Development in Mathematics and Applications, \\ University of Aveiro, Campus Universitário de Santiago, 3810-193 Aveiro, Portugal.
}

Received: 6 May 2020; Accepted: 30 May 2020; Published: 3 June 2020

check for updates

\begin{abstract}
In this article, we prove an orthogonal decomposition theorem for real inner product gyrogroups, which unify some well-known gyrogroups in the literature: Einstein, Möbius, Proper Velocity, and Chen's gyrogroups. This leads to the study of left (right) coset partition of a real inner product gyrogroup induced from a subgyrogroup that is a finite dimensional subspace. As a result, we obtain gyroprojectors onto the subgyrogroup and its orthogonal complement. We construct also quotient spaces and prove an associated isomorphism theorem. The left (right) cosets are characterized using gyrolines (cogyrolines) together with automorphisms of the subgyrogroup. With the algebraic structure of the decompositions, we study fiber bundles and sections inherited by the gyroprojectors. Finally, the general theory is exemplified for the aforementioned gyrogroups.
\end{abstract}

Keywords: real inner product gyrogroup; orthogonal decomposition; gyroprojection; coset space; partitions; quotient space; gyrolines; cogyrolines; fiber bundles

MSC: Primary 20N05; Secondary 15A63

\section{Introduction}

Gyrogroups are a suitable generalization of groups, arising from the study of the parametrization of the Lorentz transformation group made by Ungar in [1]. A vast and comprehensive collection of results about gyrogroup theory was compiled by Ungar in the books [2] and [3] (see also the vast list of references therein). Gyrogroups share remarkable analogies with groups. In fact, every group forms a gyrogroup under the same operation. The three main gyrogroups associated with analytic hyperbolic geometry are Möbius, Einstein, and Proper Velocity gyrogroups (see [3]). Each of them has a gyrovector space structure that enables to treat analytic hyperbolic geometry similarly as in the Euclidean case. Factorizations of Möbius gyrogroups were first studied in [4,5]. These factorizations were used for defining continuous wavelet transforms on the unit sphere, associated to appropriate sections in the quotient Möbius gyrogroup [6]. This makes use of strong connections between Möbius transformations, Clifford algebras, and gyrogroups. It can be seen that the gyrogroup structure is important in order to factorize the unit ball of $n$-dimensional Euclidean space $\mathbb{R}^{n}$ and to obtain sections that allow defining dilation operators on the unit sphere ([6], Section 4). Recently, in [7], the authors rediscovered gyrodecompositions in the Möbius gyrogroup and incorporated the gyrovector structure in their study, however, they did not refer to previous results obtained in [5]. Using the notion of L-subgyrogroups, Suksumran and Wiboonton studied the factorization of abstract gyrogroups, similar 
to the case of groups [8], and applied the theory to a finite gyrogroup. As it is shown in this paper, their theory does not apply here.

The aim of this paper is to give a general theory for real inner product gyrogroups that encode a real gyrovector space structure imposing only two conditions (see Section 3). In this way, we unify the factorization theory for some well-known gyrogroups in the literature that are examples of real inner product gyrogroups such as Einstein, Möbius, Proper Velocity, and Chen's gyrogroups. Our results can be used for constructing orthogonal gyroexpansions with respect to any orthogonal basis in a real inner product gyrogroup, or in the construction of integral transforms such as the wavelet transform on some manifolds, such as the sphere, the ball, or the hyperboloid.

The structure of the article is organized as follows. In Section 2, we review the basic theory of gyrogroups and gyrovector spaces. In Section 3, we examine orthogonal decompositions of real inner product gyrogroups and construct left and right coset spaces, gyroprojectors, quotient gyrogroups, and give a geometric realization of the cosets using gyrolines and cogyrolines of the gyrogroup. In Section 4, we examine fiber bundles and sections of real inner product gyrogroups inherited by the gyroprojectors. In Section 5, we present concrete prominent examples of real inner product gyrogroups: Einstein, Möbius, Proper Velocity, and Chen's gyrogroups and state several theorems obtained as consequences of the general theorems given in previous sections.

\section{Preliminaries}

Definition 1 (Gyrogroups). A nonempty set $G$, together with a binary operation $\oplus$ on $G$, is called a gyrogroup if it satisfies the following axioms.

(G1) There exists an element $0 \in G$ such that $0 \oplus a=a$ for all $a \in G$.

(G2) For each $a \in G$, there exists an element $b \in G$ such that $b \oplus a=0$.

(G3) For all $a, b \in G$, there is an automorphism $\operatorname{gyr}[a, b] \in \operatorname{Aut} G$ such that

$$
a \oplus(b \oplus c)=(a \oplus b) \oplus \operatorname{gyr}[a, b] c
$$

(left gyroassociative law)

for all $c \in G$.

(G4) For all $a, b \in G, \operatorname{gyr}[a \oplus b, b]=\operatorname{gyr}[a, b]$.

(left loop property)

It can be proved that the element 0 in (G1) is also a right identity and is unique, called the gyrogroup identity. Further, the element $b$ in (G2) is also a right inverse of $a$ and is unique, called the inverse of $a$, written $\ominus a$. A gyrogroup $(G, \oplus)$ is said to be gyrocommutative if it satisfies the following gyrocommutative law:

$$
a \oplus b=\operatorname{gyr}[a, b](b \oplus a)
$$

for all $a, b \in G$.

To capture useful analogies between gyrogroups and groups there is defined as a second binary operation in $G$, called the coaddition, which is denoted by $\boxplus$ and given by

$$
a \boxplus b=a \oplus \operatorname{gyr}[a, \ominus b] b
$$

for all $a, b \in G$ (cf. Definition 2.7 of [2]). We have also that $a \boxminus b=a \boxplus(\ominus b)$.

The main cancellation laws in a gyrogroup $(G, \oplus)$ are

$$
\begin{array}{ll}
a \oplus(\ominus a \oplus b)=b & \text { left cancellation law } \\
(b \ominus a) \boxplus a=b & \text { first right cancellation law } \\
(b \boxminus a) \oplus a=b & \text { second right cancellation law. }
\end{array}
$$

Using the left gyroassociative law given in (G3) and the left cancellation law (3) we can write the following gyrator identity 


$$
\operatorname{gyr}[a, b] c=\ominus(a \oplus b) \oplus(a \oplus(b \oplus c))
$$

from which it follows that $\operatorname{gyr}[a, b] 0=0$, i.e., gyrations are gyroautomorphisms of $G$ that preserve the identity element.

The next theorem shows the unique solution of the two basic gyrogroup equations.

Theorem 1. (see [2]) Let $(G, \oplus)$ be a gyrogroup, and let $a, b \in G$. The unique solution of the equation $a \oplus x=b$ is $x=\ominus a \oplus b$, and the unique solution of the equation $x \oplus a=b$ is $x=b \boxminus a$.

Definition 2 (Real inner product gyrovector spaces, [2]). A real inner product gyrovector space $(G, \oplus, \otimes)$ (gyrovector space, in short) is a gyrocommutative gyrogroup $(G, \oplus)$ that obeys the following axioms:

(A1) $G$ is a subset of a real inner product vector space $V, G \subseteq V$, from which it inherits its inner product and norm $\|\cdot\|$, which are invariant under gyroautomorphisms; that is,

$$
\operatorname{gyr}[u, v] a \cdot \operatorname{gyr}[u, v] b=a \cdot b
$$

for all $a, b, u, v \in G$.

(A2) $G$ admits a scalar multiplication $\otimes$ satisfying for all $r, r_{1}, r_{2} \in \mathbb{R}, a \in G$,

(V1) $1 \otimes a=a$

(V2) $\quad\left(r_{1}+r_{2}\right) \otimes a=r_{1} \otimes a \oplus r_{2} \otimes a$

(V3) $\quad\left(r_{1} r_{2}\right) \otimes a=r_{1} \otimes\left(r_{2} \otimes a\right)$

(V4) $\frac{|r| \otimes a}{\|r \otimes a\|}=\frac{a}{\|a\|}$

(V5) $\operatorname{gyr}[u, v](r \otimes a)=r \otimes \operatorname{gyr}[u, v] a$

(V6) $\operatorname{gyr}\left[r_{1} \otimes v, r_{2} \otimes v\right]=I, I$ is the identity map

(A3) The set $\|G\|=\{ \pm\|a\|: a \in G\}$ admits a real vector space structure $(\|G\|, \oplus, \otimes)$ such that

(V7) $\quad\|r \otimes a\|=|r| \otimes\|a\|$

(V8) $\quad\|a \oplus b\| \leq\|a\| \oplus\|b\|$

for all $a, b \in G, r \in \mathbb{R}$.

Unlike in the vector case, gyroaddition $\oplus$ does not in general distribute with scalar multiplication. However, gyrovector spaces possess

a monodistributive law given by

$$
r \otimes\left(r_{1} \otimes a \oplus r_{2} \otimes a\right)=r \otimes\left(r_{1} \otimes a\right) \oplus r \otimes\left(r_{2} \otimes a\right)
$$

for all $r, r_{1} r_{2} \in \mathbb{R}, a \in G$.

The motions of a gyrovector space $(G, \oplus, \otimes)$ are all its left gyrotranslations $L_{a}: x \mapsto a \oplus x$, where $a, x \in G$, and its automorphisms $\tau \in \operatorname{Aut}(G, \oplus, \otimes)$ satisfying

$$
\begin{aligned}
\tau(a \oplus b) & =\tau(a) \oplus \tau(b) \\
\tau(r \otimes a) & =r \otimes \tau(a) \\
\tau(a \cdot b) & =\tau(a) \cdot \tau(b)
\end{aligned}
$$

for all $a, b \in G, r \in \mathbb{R}$. 
A gyrovector space is a gyrometric space with a gyrodistance given by

$$
d_{\oplus}(a, b)=\|b \ominus a\|, \quad \forall a, b \in G
$$

that satisfies the gyrotriangle inequality (see Theorem 6.9 in [2]):

$$
\|c \ominus a\| \leq\|c \ominus b\| \oplus\|b \ominus a\|, \quad \forall a, b, c \in G .
$$

Moreover, a gyrovector space is also cogyrometric with a cogyrodistance $d_{\boxplus}(a, b)$ given by

$$
d_{\boxplus}(a, b)=\|b \boxminus a\|, \quad \forall a, b \in G
$$

that satisfies the cogyrotriangle inequality (see Theorem 6.11 in [2]):

$$
\|a \boxminus \operatorname{gyr}[a \boxminus b, b \boxminus c] c\| \leq\|a \boxminus b\| \boxplus\|b \boxminus c\|, \quad \forall a, b, c \in G .
$$

Curves on which the gyrotriangle inequality reduces to an equality are called gyrolines, while curves on which the cogyrotriangle inequality reduces to an equality are called cogyrolines.

Gyrolines and cogyrolines play an important role in hyperbolic analytic geometry regulated by the gyrovector space structure. For distinct elements $a$ and $b$ in $G$, the two distinct hyperbolic line expressions are defined by

$$
\begin{array}{ll}
L^{g}=\{a \oplus(t \otimes b): t \in \mathbb{R}\} & \text { Gyroline or the hyperbolic line, } \\
L^{c}=\{(t \otimes b) \oplus a: t \in \mathbb{R}\} & \text { Cogyroline or the hyperbolic dual line. }
\end{array}
$$

Gyrolines and cogyrolines are uniquely determined by any two distinct points contained by them. Therefore, expressions in (14) can be replaced by

$$
\begin{array}{ll}
L_{a, b}^{g}=\{a \oplus(t \otimes(\ominus a \oplus b)): t \in \mathbb{R}\} & \text { Gyroline passing through } a \text { at } t=0 \text { and } b \text { at } t=1, \\
L_{a, b}^{c}=\{(t \otimes(b \boxminus a)) \oplus a: t \in \mathbb{R}\} & \text { Cogyroline passing through } a \text { at } t=0 \text { and } b \text { at } t=1 .
\end{array}
$$

Several properties of gyrolines and cogyrolines are discussed in ([2], Chapter 6). Among them we mention the following properties of gyrolines:

P1. Two gyrolines that share two distinct points are coincident. (Theorem 6.20)

P2. A left gyrotranslation of a gyroline is again a gyroline. (Theorem 6.21)

P3. For $\tau \in \operatorname{Aut}(G)$, it holds that $\tau(a \oplus(t \otimes b))=\tau a \oplus(t \otimes \tau b)$. (Theorem 6.37)

P4. Gyrolines are gyrogeodesics. (Theorem 6.50)

Cogyrolines are natural objects in gyrovector spaces and also have important properties. We mention the following:

P5. Two cogyrolines that share two distinct points are coincident. (Theorem 6.53)

P6. Cogyrolines admit parallelism. (Theorem 6.65)

P7. The cogyrotranslation $\boxminus d$ of the cogyroline $(t \otimes b) \oplus a$ is again a cogyroline, that is, $((t \otimes b) \oplus$ $a) \boxminus d$ is a cogyroline. Moreover, the cogyroline and the cogyrotranslated cogyroline are parallel. (Theorem 6.66)

P8. Cogyrolines are cogyrogeodesics. (Theorem 6.78)

For the basic theory of gyrogroups and gyrovector spaces the interested reader is referred to $[2,3,9]$. Next we present the definition of subgyrogroups and L-subgyrogroups. 
Definition 3. Ref. [8] Let $(G, \oplus)$ be a gyrogroup. A nonempty subset $H$ of $G$ is called a subgyrogroup, denoted by $H \leq G$, if $H$ forms a gyrogroup under the restriction of the operation inherited from $G$ and for all $a, b \in H$, the restriction of $\operatorname{gyr}[a, b]$ to $H$ is an automorphism of $H$.

Theorem 2. Ref. [8] (The subgyrogroup criterion) A nonempty subset $H$ of $G$ is a subgyrogroup if and only if $a \oplus b \in H$ and $\ominus a \in H$ for all $a, b \in H$.

The notion of L-subgyrogroups was introduced in [8].

Definition 4. A subgyrogroup $H$ of $G$ is said to be an L-subgyrogroup, denoted by $H \leq_{L} G$, if gyr $[a, h] H=H$ for all $a \in G$ and $h \in H$.

For more details about factorization of gyrogroups by L-subgyrogroups, Cayley's Theorem, and isomorphisms theorems, see [8].

\section{Orthogonal Decompositions}

Trying to be as general as possible we define next a real inner product gyrogroup contained in a real inner product space using the general addition (15).

Definition 5 (Real inner product gyrogroups). A gyrogroup $(G, \oplus)$ contained in a real inner product space $(V,+, \cdot)$ is called a real inner product gyrogroup if it contains the zero of $V$ as the gyrogroup identity and the binary operation $\oplus$ is given by

$$
a \oplus b=\phi_{1}(a, b) a+\phi_{2}(a, b) b
$$

for all $a, b \in G$, where $\phi_{i}: G \times G \rightarrow \mathbb{R}$ is a map with $\phi_{i}(a, \ominus a) \neq 0$ for all $a \in G, i=1,2$, and $\oplus$ satisfies the gyrogroup axioms. Moreover, we say that the real inner product gyrogroup $(G, \oplus)$ admits orthogonal decompositions if the following additional conditions are satisfied:

(H1) For all $v \in V$, if $\|v\| \leq\|a\|$ for some $a \in G$, then $v \in G$.

(H2) For all $c_{1}, c_{2} \in G$ such that $c_{1}+c_{2} \in G$ and $c_{1} \cdot c_{2}=0$, there exist nonzero scalars $\lambda_{1}=\lambda_{1}\left(c_{1}, c_{2}\right) \in \mathbb{R}$ and $\lambda_{2}=\lambda_{2}\left(c_{1}, c_{2}\right) \in \mathbb{R}$ such that $\left(x=\lambda_{1} c_{1}, y=\lambda_{2} c_{2}\right) \in G \times G$ is the unique solution of the system of equations:

$$
\left\{\begin{array}{l}
\phi_{1}(x, y) x=c_{1} \\
\phi_{2}(x, y) y=c_{2}
\end{array} .\right.
$$

We remark that since the general addition $a \oplus b$ defined in (15) satisfies the gyrogroup axioms of Definition 1 then the functions $\phi_{1}$ and $\phi_{2}$ have to satisfy some functional equalities and relations. In particular, since, $0 \oplus b=b$ and $a \oplus 0=a$, for all $a, b \in G$, then it implies that $\phi_{1}(a, 0)=1$ and $\phi_{2}(0, b)=1$, for all $a, b \in G$. Regarding the conditions (H1) and (H2), the first condition says essentially that $G$ consists of a ball with finite or infinite radius in the carrier space $V$, while the second condition allows us to obtain orthogonal decompositions of $G$, and corresponding left and right cosets of $G$. Concrete prominent examples of real inner product gyrogroups that admit orthogonal decompositions are exhibited in Section 5: Euclidean Einstein, Möbius, Proper Velocity, and Chen's gyrogroups.

Throughout the remainder of this article, we assume that $(G, \oplus)$ is a real inner product gyrogroup contained in the carrier inner product space $(V,+, \cdot)$ satisfying conditions $(\mathrm{H} 1)$ and $(\mathrm{H} 2)$.

\subsection{Unique Decomposition and Orthogonal Gyroprojections}

Let $P$ be a finite-dimensional linear subspace of $V$ and let $P^{\perp}$ be its orthogonal complement in $V$ (that is, $V=P \oplus P^{\perp}$ is an orthogonal direct sum). Define

$$
P_{G}=P \cap G \quad \text { and } \quad P_{G}^{\perp}=P^{\perp} \cap G .
$$

Theorem 3. If $P$ is a finite-dimensional subspace of $V$, then $P_{G}$ and $P_{G}^{\perp}$ are subgyrogroups of $G$. 
Proof. Note that $P_{G} \neq \varnothing$ since $0 \in P_{G}$. Let $a, b \in P_{G}$. Then $a, b \in P$ and $a, b \in G$. By (15), $a \oplus b \in P$ since $P$ is closed under vector addition. By the closure property of $G, a \oplus b \in G$. Hence, $a \oplus b \in P_{G}$. Note that $0=a \oplus(\ominus a)=\phi_{1}(a, \ominus a) a+\phi_{2}(a, \ominus a) \ominus a$, which implies $\ominus a=-\frac{\phi_{1}(a, \ominus a)}{\phi_{2}(a, \ominus a)} a$ since $\phi_{2}(a, \ominus a) \neq 0$. Hence, $\ominus a \in P$ and so $\ominus a \in P_{G}$. This proves that $P_{G}$ is a subgyrogroup of $G$. Since the proof does not make use of the assumption of being finite-dimensional, we conclude that $P_{G}^{\perp}$ is a subgyrogroup of $G$ as well.

According to Definition $4, P_{G}$ is an L-subgyrogroup if $\operatorname{gyr}[a, h] P_{G}=P_{G}$, for all $a \in G$ and $h \in P_{G}$. Since gyrations are isometries of $(G, \oplus, \otimes)$ that preserve the norm inherited from $(V,+, \cdot)$, the only gyrations that preserve $P_{G}$ are from the automorphism group of $P_{G}$. Hence, $P_{G}$ is not an L-subgyrogroup.

A first fundamental result that we are going to show is the conditions under which the addition $\oplus$ in a real inner product gyrogroup is associative.

Theorem 4. Let $a, b, c \in(G, \oplus, \otimes)$. Then $a \oplus(b \oplus c)=(a \oplus b) \oplus c$ if and only if $a \cdot c=0$ and $b \cdot c=0$ or $a=\lambda \otimes b$ for some $\lambda \in \mathbb{R}$.

Proof. By the left gyroassociative law we have

$$
a \oplus(b \oplus c)=(a \oplus b) \oplus \operatorname{gyr}[a, b] c .
$$

We know that isometries of a real inner product space $(V,+, \cdot)$ that fix the origin and an arbitrary element $c$ are the isometries that belong to a linear subspace orthogonal to $c$ or the trivial isometry (identity isometry) that belongs to a one-dimensional linear subspace of $V$. Therefore, since gyrations of $(G, \oplus, \otimes)$ are isometries in the carrier space $V$, it follows that $\operatorname{gyr}[a, b] c=c$ if and only if $a \cdot c=0$ and $b \cdot c=0$ or $a=\lambda \otimes b$ for some $\lambda \in \mathbb{R}$, i.e., either $a$ and $b$ belong to a linear subspace orthogonal to $c$ or $a$ and $b$ are in a given one dimensional linear subspace. Note that by axiom (V6) in Definition 2 it follows that $\operatorname{gyr}[a, \lambda \otimes a]=I$.

The following theorem shows that any real inner product gyrogroup $G$ can be decomposed into gyrosums of $P_{G}$ and $P_{G}^{\perp}$, where $P$ is a finite-dimensional subspace of the carrier space $V$.

Theorem 5 (Unique Decomposition). Let $P$ be a finite-dimensional subspace of $V$. For all $c \in G$, there are unique elements $a, u \in P_{G}, b, v \in P_{G}^{\perp}$ such that

$$
c=a \oplus b \quad \text { and } \quad c=v \oplus u .
$$

Proof. Let $c \in G$ be arbitrary. Since $G \subseteq V$ and $V=P \oplus P^{\perp}$, it follows that $c=c_{1}+c_{2}$ with $c_{1} \in P$ and $c_{2} \in P^{\perp}$. Note that $\|c\|^{2}=\left\|c_{1}+c_{2}\right\|^{2}=\left\|c_{1}\right\|^{2}+\left\|c_{2}\right\|^{2}$. Hence, $\left\|c_{1}\right\| \leq\|c\|$ and $\left\|c_{2}\right\| \leq\|c\|$. By hypothesis (H1), $c_{1} \in G$ and $c_{2} \in G$. Since $c_{1}+c_{2}=c \in G$ and $c_{1} \cdot c_{2}=0$, we have by hypothesis (H2) that there exist scalars $\lambda_{1}=\lambda_{1}\left(c_{1}, c_{2}\right), \lambda_{2}=\lambda_{2}\left(c_{1}, c_{2}\right) \in \mathbb{R}$ such that $\left(x=\lambda_{1} c_{1}, y=\lambda_{2} c_{2}\right) \in G \times G$ is the unique solution of the system of equations:

$$
\left\{\begin{array}{l}
\phi_{1}(x, y) x=c_{1} \\
\phi_{2}(x, y) y=c_{2}
\end{array} .\right.
$$

Set $a=\lambda_{1} c_{1}$ and $b=\lambda_{2} c_{2}$. Then $a \in P_{G}$ and $b \in P_{G}^{\perp}$. Furthermore, we obtain

$$
\begin{aligned}
a \oplus b & =\phi_{1}(a, b) a+\phi_{2}(a, b) b \\
& =\phi_{1}\left(\lambda_{1} c_{1}, \lambda_{2} c_{2}\right) \lambda_{1} c_{1}+\phi_{2}\left(\lambda_{1} c_{1}, \lambda_{2} c_{2}\right) \lambda_{2} c_{2} \\
& =c_{1}+c_{2} \\
& =c .
\end{aligned}
$$


To prove uniqueness of the factorization, suppose that $c=a^{\prime} \oplus b^{\prime}$ with $a^{\prime} \in P_{G}, b^{\prime} \in P_{G}^{\perp}$. Then

$$
c_{1}+c_{2}=\phi_{1}\left(a^{\prime}, b^{\prime}\right) a^{\prime}+\phi_{2}\left(a^{\prime}, b^{\prime}\right) b^{\prime}
$$

and so $c_{1}=\phi_{1}\left(a^{\prime}, b^{\prime}\right) a^{\prime}$ and $c_{2}=\phi_{2}\left(a^{\prime}, b^{\prime}\right) b^{\prime}$ since the sum $P \oplus P^{\perp}$ is direct. It follows that $\left(a^{\prime}, b^{\prime}\right)$ is a solution of (19). Hence, $a^{\prime}=\lambda_{1} c_{1}=a$ and $b^{\prime}=\lambda_{2} c_{2}=b$.

Similarly, $c_{2}+c_{1}=c \in G$ and $c_{2} \cdot c_{1}=0$ together imply by hypothesis (H2) that there exist scalars $\lambda_{3}=\lambda_{3}\left(c_{1}, c_{2}\right) \in \mathbb{R}$ and $\lambda_{4}=\lambda_{4}\left(c_{1}, c_{2}\right) \in \mathbb{R}$ such that $\left(x=\lambda_{3} c_{2}, y=\lambda_{4} c_{1}\right) \in G \times G$ is the unique solution of the system of equations (19). Regarding the first decomposition it is immediate to see that $\lambda_{3}\left(c_{1}, c_{2}\right)=\lambda_{1}\left(c_{2}, c_{1}\right)$ and $\lambda_{4}\left(c_{1}, c_{2}\right)=\lambda_{2}\left(c_{2}, c_{1}\right)$, with the order of $c_{1}$ and $c_{2}$ being changed in $\lambda_{1}$ and $\lambda_{2}$. Set $v=\lambda_{3} c_{2}$ and $u=\lambda_{4} c_{1}$. Then $u \in P_{G}, v \in P_{G}^{\perp}$, and $c=v \oplus u$. Uniqueness of the factorization can be proved in the same way.

We remark that the proof of the uniqueness of Theorem 5 could also be made using Theorem 4 .

Corollary 1 (Orthogonal Decompositions). The following orthogonal decompositions of $G$ hold:

$$
G=P_{G} \oplus P_{G}^{\perp} \quad \text { and } \quad G=P_{G}^{\perp} \oplus P_{G} .
$$

Using Theorem 5 , we can define orthogonal gyroprojections of the gyrogroup $(G, \oplus)$ onto the subgyrogroups $P_{G}$ and $P_{G}^{\perp}$, with respect to the decompositions $G=P_{G} \oplus P_{G}^{\perp}$ and $G=P_{G}^{\perp} \oplus P_{G}$. In fact, each element $c \in G$ admits the unique decomposition $c=c_{1}+c_{2}=c_{2}+c_{1}$, with $c_{1} \in P_{G}$ and $c_{2} \in P_{G}^{\perp}$ and according to Theorem 5 four orthogonal gyroprojectors can be defined:

$$
\begin{array}{ll}
\mathcal{P}^{\ell}: G=P_{G} \oplus P_{G}^{\perp} \rightarrow P_{G}, & \mathcal{P}^{\ell}(c)=\lambda_{1}\left(c_{1}, c_{2}\right) c_{1}, \\
\mathcal{Q}^{r}: G=P_{G} \oplus P_{G}^{\perp} \rightarrow P_{G}^{\perp}, & \mathcal{Q}^{r}(c)=\lambda_{2}\left(c_{1}, c_{2}\right) c_{2}, \\
\mathcal{Q}^{\ell}: G=P_{G}^{\perp} \oplus P_{G} \rightarrow P_{G}^{\perp}, & \mathcal{Q}^{\ell}(c)=\lambda_{3}\left(c_{1}, c_{2}\right) c_{2}=\lambda_{1}\left(c_{2}, c_{1}\right) c_{2}, \\
\mathcal{P}^{r}: G=P_{G}^{\perp} \oplus P_{G} \rightarrow P_{G}, & \mathcal{P}^{r}(c)=\lambda_{4}\left(c_{1}, c_{2}\right) c_{1}=\lambda_{2}\left(c_{2}, c_{1}\right) c_{1},
\end{array}
$$

such that

$$
c=\mathcal{P}^{\ell}(c) \oplus \mathcal{Q}^{r}(c) \quad \text { and } \quad c=\mathcal{Q}^{\ell}(c) \oplus \mathcal{P}^{r}(c),
$$

where the superscripts $\ell$ and $r$ stand for "left" and "right" and indicate that the gyroprojection is on the left or on the right of each decomposition. The following identities hold immediately:

$$
\left(\mathcal{P}^{\ell}\right)^{2}=\mathcal{P}^{\ell}, \quad\left(\mathcal{P}^{r}\right)^{2}=\mathcal{P}^{r}, \quad\left(\mathcal{Q}^{\ell}\right)^{2}=\mathcal{Q}^{\ell}, \quad\left(\mathcal{Q}^{r}\right)^{2}=\mathcal{Q}^{r} .
$$

Moreover, we obtain the following orthogonal gyrodecompositions:

$$
\mathcal{I}=\mathcal{P}^{\ell} \oplus \mathcal{Q}^{r} \quad \text { and } \quad \mathcal{I}=\mathcal{Q}^{\ell} \oplus \mathcal{P}^{r} .
$$

Corollary 2. For all $a \in P_{G}$ and $b \in P_{G}^{\perp}$, we have

$$
\begin{array}{llll}
\mathcal{P}^{\ell}(a \oplus b)=a & \text { and } & \mathcal{Q}^{r}(a \oplus b)=b, \\
\mathcal{P}^{r}(b \oplus a)=a & \text { and } & \mathcal{Q}^{\ell}(b \oplus a)=b .
\end{array}
$$

Proof. By (15) and Theorem 5 we have

$$
a \oplus b=\phi_{1}(a, b) a+\phi_{2}(a, b) b=\lambda_{1}\left(\phi_{1}(a, b) a, \phi_{2}(a, b) b\right) \phi_{1}(a, b) a \oplus \lambda_{2}\left(\phi_{1}(a, b) a, \phi_{2}(a, b) b\right) \phi_{2}(a, b) b
$$


which implies by the Unique Decomposition Theorem (Theorem 5) that

$$
\left\{\begin{array}{l}
\lambda_{1}\left(\phi_{1}(a, b) a, \phi_{2}(a, b) b\right) \phi_{1}(a, b) a=a \\
\lambda_{2}\left(\phi_{1}(a, b) a, \phi_{2}(a, b) b\right) \phi_{2}(a, b) b=b
\end{array} .\right.
$$

Hence, using the definition of the gyroprojections (20) and (25) we obtain the identities

$$
\mathcal{P}^{\ell}(a \oplus b)=a \quad \text { and } \quad \mathcal{Q}^{r}(a \oplus b)=b .
$$

Using similar reasonings we obtain also

$$
\mathcal{P}^{r}(b \oplus a)=a \quad \text { and } \quad \mathcal{Q}^{\ell}(b \oplus a)=b .
$$

By Corollary 1 the gyrogroup $(G, \oplus)$ has two unique decompositions $G=P_{G} \oplus P_{G}^{\perp}$ and $G=P_{G}^{\perp} \oplus P_{G}$. The relation between them is given more precisely in the next theorem.

Theorem 6. Let $a, b \in(G, \oplus)$ such that $a \cdot b=0$. Then there exist nonzero scalars $\mu_{1}(a, b)$ and $\mu_{2}(a, b)$ such that

$$
a \oplus b=\mu_{1}(a, b) b \oplus \mu_{2}(a, b) a .
$$

Proof. Let $a, b \in G$ such that $a \perp b$. Then by (15) we have

$$
a \oplus b=\phi_{1}(a, b) a+\phi_{2}(a, b) b .
$$

Consider now $c_{1}=\phi_{1}(a, b) a$ and $c_{2}=\phi_{2}(a, b) b$. Then $c_{1} \perp c_{2}$ and by Theorem 5 there exist nonzero scalars $\lambda_{3}=\lambda_{3}\left(c_{1}, c_{2}\right) \in \mathbb{R}$ and $\lambda_{4}=\lambda_{4}\left(c_{1}, c_{2}\right) \in \mathbb{R}$ such that

$$
\begin{aligned}
a \oplus b & =\lambda_{3}\left(c_{1}, c_{2}\right) c_{2} \oplus \lambda_{4}\left(c_{1}, c_{2}\right) c_{1} \\
& =\lambda_{3}\left(\phi_{1}(a, b) a, \phi_{2}(a, b) b\right) \phi_{2}(a, b) b+\lambda_{4}\left(\phi_{1}(a, b) a, \phi_{2}(a, b) b\right) \phi_{1}(a, b) a .
\end{aligned}
$$

Taking

$$
\left\{\begin{array}{l}
\mu_{1}(a, b)=\lambda_{3}\left(\phi_{1}(a, b) a, \phi_{2}(a, b) b\right) \phi_{2}(a, b) \\
\mu_{2}(a, b)=\lambda_{4}\left(\phi_{1}(a, b) a, \phi_{2}(a, b) b\right) \phi_{1}(a, b)
\end{array}\right.
$$

we obtain (26).

Corollary 3. For all $a \in P_{G}$ and $b \in P_{G}^{\perp}$, we have

$$
\begin{array}{lll}
\mathcal{P}^{\ell}(b \oplus a)=\mu_{1}(b, a) a & \text { and } & \mathcal{Q}^{r}(b \oplus a)=\mu_{2}(b, a) b, \\
\mathcal{P}^{r}(a \oplus b)=\mu_{2}(a, b) a & \text { and } & \mathcal{Q}^{\ell}(a \oplus b)=\mu_{1}(a, b) b .
\end{array}
$$

Proof. From Theorem 6 we have:

$$
\begin{aligned}
& \mathcal{P}^{\ell}(b \oplus a)=\mathcal{P}^{\ell}\left(\mu_{1}(b, a) a \oplus \mu_{2}(b, a) b\right)=\mu_{1}(b, a) a, \\
& \mathcal{Q}^{r}(b \oplus a)=\mathcal{Q}^{r}\left(\mu_{1}(b, a) a \oplus \mu_{2}(b, a) b\right)=\mu_{2}(b, a) b, \\
& \mathcal{P}^{r}(a \oplus b)=\mathcal{P}^{r}\left(\mu_{1}(a, b) b \oplus \mu_{2}(a, b) a\right)=\mu_{2}(a, b) a, \\
& \mathcal{Q}^{\ell}(a \oplus b)=\mathcal{Q}^{\ell}\left(\mu_{1}(a, b) b \oplus \mu_{2}(a, b) a\right)=\mu_{1}(a, b) b .
\end{aligned}
$$

\subsection{Left and Right Coset Spaces}

Let $H$ be a subgyrogroup of $G$. In contrast to groups, the relation

$$
a \sim b \quad \text { if and only if } \quad \ominus a \oplus b \in H
$$


does not, in general, define an equivalence relation on $G$. In [8] this relation was modified to

$$
a \sim b \quad \text { if and only if } \quad \ominus a \oplus b \in H \text { and } \operatorname{gyr}[\ominus a, b] H=H
$$

obtaining an equivalence relation on $G$ and leading to the notion of L-subgyrogroups. Since in our case $P_{G}$ is not an L-subgyrogroup, we cannot apply the results in [8]. In the case of real inner product gyrogroups that have attached a gyrovector space structure we are going to show that the gyroprojection $\mathcal{Q}^{\ell}$ (respectively, $\mathcal{Q}^{r}$ ) induces an equivalence relation on $G$ so that $G$ can be written as a disjoint union of left (respectively, right) cosets with representatives from $P_{G}^{\perp}$.

Let $P$ be a finite-dimensional linear subspace of $V$. Define $\sim_{\ell}$ by

$$
a \sim_{\ell} b \quad \text { if and only if } \quad \ominus \mathcal{Q}^{\ell}(b) \oplus a \in P_{G}
$$

for all $a, b \in G$.

Theorem 7. The relation $\sim_{\ell}$ defined by (29) is an equivalence relation on $G$.

Proof. Let $a, b, c \in G$.

(Reflexive property) Since $a=\mathcal{Q}^{\ell}(a) \oplus \mathcal{P}^{r}(a)$, it follows from Theorem 1 we have $\ominus \mathcal{Q}^{\ell}(a) \oplus a=$ $\mathcal{P}^{r}(a) \in P_{G}$. Hence, $a \sim_{\ell} a$.

(Symmetric property) Suppose that $a \sim_{\ell} b$. Then $\ominus \mathcal{Q}^{\ell}(b) \oplus a \in P_{G}$ and so there is an element $c \in P_{G}$ such that $\ominus \mathcal{Q}^{\ell}(b) \oplus a=c$. This implies $a=\mathcal{Q}^{\ell}(b) \oplus c$. It follows that $\mathcal{Q}^{\ell}(a)=\mathcal{Q}^{\ell}\left(\mathcal{Q}^{\ell}(b) \oplus c\right)=\mathcal{Q}^{\ell}(b)$. Since $b=Q^{\ell}(b) \oplus P^{r}(b)$, then we obtain from Theorem 1 that $\ominus Q^{\ell}(b) \oplus b=P^{r}(b)$. Therefore,

$$
\ominus Q^{\ell}(a) \oplus b=\ominus Q^{\ell}(b) \oplus b=P^{r}(b) \in P_{G} .
$$

Hence, $b \sim_{\ell} a$.

(Transitive property) Suppose that $a \sim_{\ell} b$ and $b \sim_{\ell} c$. It follows that $\mathcal{Q}^{\ell}(a)=\mathcal{Q}^{\ell}(b)$ and $\mathcal{Q}^{\ell}(b)=\mathcal{Q}^{\ell}(c)$, as proved in the previous paragraph. Since $\ominus \mathcal{Q}^{\ell}(a) \oplus a=\mathcal{P}^{r}(a)$, we obtain that $\ominus \mathcal{Q}^{\ell}(c) \oplus a=\ominus \mathcal{Q}^{\ell}(a) \oplus a=\mathcal{P}^{r}(a)$ belongs to $P_{G}$. Hence, $a \sim_{\ell} c$.

For each $b \in G$, we denote by $[b]_{\ell}$ the equivalence class containing $b$ determined by the relation $\sim_{\ell}$.

Lemma 1. For all $b \in G,[b]_{\ell}=Q^{\ell}(b) \oplus P_{G}$, where

$$
Q^{\ell}(b) \oplus P_{G}=\left\{Q^{\ell}(b) \oplus u: u \in P_{G}\right\} .
$$

Proof. Let $x \in Q^{\ell}(b) \oplus P_{G}$. Then $x=Q^{\ell}(b) \oplus u$ for some $u \in P_{G}$. By the left cancellation law we have $\ominus Q^{\ell}(b) \oplus x=u \in P_{G}$, that is, $x \sim_{\ell} b$, which implies $x \in[b]_{\ell}$. To prove the reverse inclusion, let $x \in[b]_{\ell}$. Then $x \sim_{\ell} b$, that is, $\ominus Q^{\ell}(b) \oplus x \in P_{G}$. Then, there exists an element $u \in P_{G}$ such that $\ominus Q^{\ell}(b) \oplus x=u$. Hence, by the left cancellation law we have $x=Q^{\ell}(b) \oplus u$, that is, $x \in Q^{\ell}(b) \oplus P_{G}$. Thus, $[b]_{\ell}=Q^{\ell}(b) \oplus P_{G}$.

Theorem 8. Let $P$ be a finite-dimensional linear subspace of $V$. Then the collection

$$
\left\{b \oplus P_{G}: b \in P_{G}^{\perp}\right\}
$$

is a disjoint partition of $G$, that is,

$$
G=\bigcup_{b \in P_{G}^{\perp}}\left(b \oplus P_{G}\right)
$$


Proof. According to Lemma 1, we know that for all $b, v \in P_{G}^{\perp}$, either $b \oplus P_{G}=v \oplus P_{G}$ or $\left(b \oplus P_{G}\right) \cap$ $\left(v \oplus P_{G}\right)=\varnothing$ since $b \oplus P_{G}=[b]_{\ell}$ and $v \oplus P_{G}=[v]_{\ell}$. Hence, the union in (30) is disjoint. It is clear that $b \oplus P_{G} \neq \varnothing$ since $b \in b \oplus P_{G}$ for all $b \in P_{G}^{\perp}$. Let $x \in G$. By Theorem $5, x=v \oplus u$ for some $v \in P_{G}^{\perp}$, $u \in P_{G}$. Hence, $x \in v \oplus P_{G} \subseteq \bigcup_{b \in P_{\bar{G}}^{\perp}}\left(b \oplus P_{G}\right)$. This proves $G \subseteq \bigcup_{b \in P_{\bar{G}}^{\perp}}\left(b \oplus P_{G}\right)$ and so equality holds.

Denote by $\left(G / P_{G}, \sim_{\ell}\right)$ the set of all equivalence classes obtained by the equivalence relation $\sim_{\ell}$. By Lemma 1 and Theorem 8 the coset space $\left(G / P_{G}, \sim_{\ell}\right)$ represents the collection $\left\{b \oplus P_{G}: b \in P_{G}^{\perp}\right\}$.

Corollary 4. The set $\left(G / P_{G}, \sim_{\ell}\right)$ is a left coset space of $G$ whose cosets are of the form $b \oplus P_{G}$ with $b \in P_{G}^{\perp}$.

Next, we present the right counterpart of Theorem 8 defining first the equivalence relation that leads to the construction of the right cosets.

Let $P$ be a finite-dimensional linear subspace of $V$. Define $\sim_{r}$ by

$$
a \sim_{r} b \quad \text { if and only if } \quad a \boxminus \mathcal{Q}^{r}(b) \in P_{G}
$$

for all $a, b \in G$, where $a \boxminus \mathcal{Q}^{r}(b)=a \boxplus\left(\ominus \mathcal{Q}^{r}(b)\right)$ and $\boxplus$ stands for the coaddition in $G$ defined in (2).

Theorem 9. The relation $\sim_{r}$ defined by (31) is an equivalence relation on $G$.

Proof. Let $a, b, c \in G$.

(Reflexive property) Since $a=\mathcal{P}^{\ell}(a) \oplus \mathcal{Q}^{r}(a)$, it follows from Theorem 1 that $\mathcal{P}^{\ell}(a)=a \boxminus \mathcal{Q}^{r}(a)$. Hence, $a \boxminus \mathcal{Q}^{r}(a) \in P_{G}$ and so $a \sim_{r} a$.

(Symmetric property) Suppose that $a \sim_{r} b$. Then $a \boxminus \mathcal{Q}^{r}(b) \in P_{G}$ and so there is an element $c \in P_{G}$ such that $a \boxminus \mathcal{Q}^{r}(b)=c$. This implies $a=c \oplus \mathcal{Q}^{r}(b)$. It follows that $\mathcal{Q}^{r}(a)=\mathcal{Q}^{r}\left(c \oplus \mathcal{Q}^{r}(b)\right)=\mathcal{Q}^{r}(b)$. Since $b=\mathcal{P}^{\ell}(b) \oplus \mathcal{Q}^{r}(b)$, we obtain from Theorem 1 that $\mathcal{P}^{\ell}(b)=b \boxminus \mathcal{Q}^{r}(b)$. Therefore, $b \boxminus Q^{r}(a)=b \boxminus Q^{r}(b)=P^{r}(b) \in P_{G}$. Hence, $b \sim_{r} a$.

(Transitive property) Suppose that $a \sim_{r} b$ and $b \sim_{r} c$. It follows that $\mathcal{Q}^{r}(a)=\mathcal{Q}^{r}(b)$ and $\mathcal{Q}^{r}(b)=\mathcal{Q}^{r}(c)$, as proved in the previous paragraph. Since $a \boxminus \mathcal{Q}^{r}(a)=\mathcal{P}^{\ell}(a)$, we obtain that

$$
a \boxminus \mathcal{Q}^{r}(c)=a \boxminus \mathcal{Q}^{r}(a)=\mathcal{P}^{\ell}(a)
$$

belongs to $P_{G}$. Hence, $a \sim_{r} c$.

For each $b \in G$, we denote by $[b]_{r}$ the equivalence class containing $b$ determined by the relation $\sim_{r}$.

Lemma 2. For all $b \in G,[b]_{r}=P_{G} \oplus Q^{r}(b)$, where

$$
P_{G} \oplus Q^{r}(b)=\left\{u \oplus Q^{r}(b): u \in P_{G}\right\} .
$$

Proof. Let $x \in P_{G} \oplus Q^{r}(b)$. Then $x=u \oplus Q^{r}(b)$ for some $u \in P_{G}$. Then, by the right cancellation law we have $x \boxminus Q^{r}(b)=u \in P_{G}$, that is, $x \sim_{r} b$, which implies $x \in[b]_{r}$. To prove the reverse inclusion let $x \in[b]_{r}$. Then $x \sim_{r} b$, that is, $x \boxminus Q^{r}(b) \in P_{G}$. Then, there exists an element $u \in P_{G}$ such that $x \boxminus Q^{r}(b)=u$. Hence, by the right cancellation law II (cf. Equation (2.63) of [3]) we have $x=u \oplus Q^{r}(b)$, that is, $x \in P_{G} \oplus Q^{r}(b)$. Thus, $[b]_{r}=P_{G} \oplus Q^{r}(b)$.

Theorem 10. Let $P$ be a finite-dimensional linear subspace of $V$. Then the collection

$$
\left\{P_{G} \oplus b: b \in P_{G}^{\perp}\right\}
$$


is a disjoint partition of $G$, that is,

$$
G=\bigcup_{b \in P_{G}^{\perp}}\left(P_{G} \oplus b\right)
$$

Proof. According to Lemma 2, we know that for all $b, v \in P_{G}^{\perp}$, either $P_{G} \oplus b=P_{G} \oplus v$ or $\left(P_{G} \oplus b\right) \cap$ $\left(P_{G} \oplus v\right)=\varnothing$ since $P_{G} \oplus b=[b]_{r}$ and $P_{G} \oplus v=[v]_{r}$. Hence, the union in (32) is disjoint. It is clear that $P_{G} \oplus b \neq \varnothing$ since $b \in P_{G} \oplus b$ for all $b \in P_{G}^{\perp}$. Let $x \in G$. By Theorem $5, x=a \oplus b$ for some $a \in P_{G}$, $b \in P_{G}^{\perp}$. Hence, $x \in P_{G} \oplus b \subseteq \bigcup_{b \in P_{\bar{G}}^{\perp}}\left(P_{G} \oplus b\right)$. This proves $G \subseteq \bigcup_{b \in P_{\bar{G}}^{\perp}}\left(P_{G} \oplus b\right)$ and so equality holds.

Denote by $\left(G / P_{G}, \sim_{r}\right)$ the set of all equivalence classes obtained by the equivalence relation $\sim_{r}$.

Corollary 5. The set $\left(G / P_{G}, \sim_{r}\right)$ is a right coset space of $G$ whose cosets are of the form $P_{G} \oplus b$ with $b \in P_{G}^{\perp}$.

\subsection{Quotient Gyrogroups and the Isomorphism Theorem}

The coset spaces $\left(G / P_{G}, \sim_{\ell}\right)$ and $\left(G / P_{G}, \sim_{r}\right)$ turn out to be gyrogroups, called left and right quotient gyrogroups by $P_{G}$, respectively.

Lemma 3. Let $P$ be an arbitrary finite-dimensional subspace of $V$.

(1) For all $a, b \in P_{G}^{\perp}, a \oplus P_{G}=b \oplus P_{G}$ if and only if $a=b$.

(2) For all $a, b \in P_{G}^{\perp}, P_{G} \oplus a=P_{G} \oplus b$ if and only if $a=b$.

Proof. Let $a, b \in P_{G}^{\perp}$.

To prove item (1), suppose that $a \oplus P_{G}=b \oplus P_{G}$. Then $a \sim_{\ell} b$ and so $\ominus \mathcal{Q}^{\ell}(b) \oplus a \in P_{G}$. Since $b \in P_{G}^{\perp}$, we have $\mathcal{Q}^{\ell}(b)=b$. Hence, $\ominus b \oplus a \in P_{G}$. Since $P_{G}^{\perp}$ is a subgyrogroup of $G$, we also have $\ominus b \oplus a \in P_{G}^{\perp}$. It follows that $\ominus b \oplus a \in P_{G} \cap P_{G}^{\perp}=\{0\}$. Hence, $\ominus b \oplus a=0$, which implies $a=b$.

To prove item (2), suppose that $P_{G} \oplus a=P_{G} \oplus b$. Then $a \sim_{r} b$ and so $a \boxminus \mathcal{Q}^{r}(b) \in P_{G}$. Since $b \in P_{G}^{\perp}$, we have $\mathcal{Q}^{r}(b)=b$. Hence, $a \boxminus b \in P_{G}$. Since $P_{G}^{\perp}$ is a subgyrogroup of $G$, we also have $a \boxminus b=a \oplus$ $\operatorname{gyr}[a, b](\ominus b) \in P_{G}^{\perp}$. It follows that $a \boxminus b \in P_{G} \cap P_{G}^{\perp}=\{0\}$. Hence, $a \boxminus b=0$, which implies $a=b$.

From Lemma 3 and the fact that $P_{G}^{\perp}$ is a subgyrogroup of $G$, we obtain that the following operations on the coset spaces $\left(G / P_{G}, \sim_{\ell}\right)$ and $\left(G / P_{G}, \sim_{r}\right)$ are well defined:

$$
\begin{array}{ll}
\left(a \oplus P_{G}\right) \oplus\left(b \oplus P_{G}\right)=(a \oplus b) \oplus P_{G}, & a, b \in P_{G}^{\perp} ; \\
\left(P_{G} \oplus a\right) \oplus\left(P_{G} \oplus b\right)=P_{G} \oplus(a \oplus b), & a, b \in P_{G}^{\perp} .
\end{array}
$$

In fact, the left and right coset spaces form gyrogroups, as shown in the following theorems.

Theorem 11. The left coset space $\left(G / P_{G}, \sim_{\ell}\right)$ forms a gyrogroup under the operation defined by (33).

Proof. The coset $0 \oplus P_{G}$ is a left identity in $G / P_{G}$. For each $a \oplus P_{G} \in G / P_{G}$ with $a \in P_{G}^{\perp}$, the coset $(\ominus a) \oplus P_{G}$ lies in $G / P_{G}$ and is a left inverse of $a \oplus P_{G}$. For $X=a \oplus P_{G}, Y=b \oplus P_{G}$ with $a, b \in P_{G}^{\perp}$, define

$$
\operatorname{gyr}[X, Y]\left(c \oplus P_{G}\right)=(\operatorname{gyr}[a, b] c) \oplus P_{G}, \quad c \in P_{G}^{\perp} .
$$

If $d \in P_{G}^{\perp}$ and if $d \oplus P_{G}=c \oplus P_{G}^{\perp}$, then $d=c$ by Lemma 3 and so

$$
(\operatorname{gyr}[a, b] d) \oplus P_{G}=(\operatorname{gyr}[a, b] c) \oplus P_{G},
$$

which proves that $\operatorname{gyr}[X, Y]$ is well defined. The proof that $\operatorname{gyr}[X, Y]$ fits the definition of a gyrogroup follows the same steps as in the proof of Theorem 29 of [9] with appropriate modifications. 
Proceeding in a similar fashion, we obtain the right counterpart of Theorem 11.

Theorem 12. The right coset space $\left(G / P_{G}, \sim_{r}\right)$ forms a gyrogroup under the operation defined by (34).

Definition 6. The gyrogroup $\left(G / P_{G}, \sim_{\ell}\right)$ in Theorem 11 is called a left quotient gyrogroup and the gyrogroup $\left(G / P_{G}, \sim_{r}\right)$ in Theorem 12 is called a right quotient gyrogroup. We define the canonical projection mappings $\pi_{\ell}$ and $\pi_{r}$ from $G$ to the left and right coset spaces by

$$
\begin{aligned}
& \pi_{\ell}(c)=[c]_{\ell}=\mathcal{Q}^{\ell}(c) \oplus P_{G}, \quad c \in G, \\
& \pi_{r}(c)=[c]_{r}=P_{G} \oplus \mathcal{Q}^{r}(c), \quad c \in G .
\end{aligned}
$$

Theorem 13 (The Isomorphism Theorem). Let $P$ be an arbitrary finite-dimensional linear subspace of $V$. Then $\pi_{\ell}$ restricts to a gyrogroup isomorphism from $P_{G}^{\perp}$ to $\left(G / P_{G}, \sim_{\ell}\right)$ and $\pi_{r}$ restricts to a gyrogroup isomorphism from $P_{G}^{\perp}$ to $\left(G / P_{G}, \sim_{r}\right)$. Therefore, the following gyrogroup isomorphisms hold:

$$
\left(G / P_{G}, \sim_{\ell}\right) \cong P_{G}^{\perp} \cong\left(G / P_{G}, \sim_{r}\right)
$$

Proof. That $\left.\pi_{\ell}\right|_{P_{\bar{G}}^{\perp}}$ is surjective is clear. That $\left.\pi_{\ell}\right|_{P_{\bar{G}}^{\perp}}$ is injective follows from Lemma 3. According to (33), $\left.\pi_{\ell}\right|_{P_{\bar{G}}^{\perp}}$ preserves the gyrogroup operations. This shows that $\left.\pi_{\ell}\right|_{P_{\bar{G}}^{\perp}}: P_{G}^{\perp} \rightarrow\left(G / P_{G}, \sim_{\ell}\right)$ is a gyrogroup isomorphism. The proof for $\left.\pi_{r}\right|_{P_{\bar{G}}^{\perp}}$ is similar. It follows that $\left(G / P_{G}, \sim_{\ell}\right) \cong P_{G}^{\perp}$ and that $\left(G / P_{G}, \sim_{r}\right) \cong P_{G}^{\perp}$.

\subsection{Geometric Characterization of Cosets}

In this section, we assume that $(G, \oplus)$ is a real inner product gyrogroup together with a scalar multiplication $\otimes$, turning $(G, \oplus, \otimes)$ a gyrovector space. The next theorem gives a geometric characterization of the left equivalence classes $b \oplus P_{G}$ with $b \in P_{G}^{\perp}$ in terms of the automorphisms of $\left(P_{G}, \oplus, \otimes\right)$ acting on a given gyroline $L^{g}$.

Theorem 14. Let $b \in P_{G}^{\perp}$ and let $0 \neq c \in P_{G}$ be fixed. Then

$$
b \oplus P_{G}=\left\{\tau L^{g}: \tau \in \operatorname{Aut}\left(P_{G}, \oplus, \otimes\right)\right\},
$$

where the gyroline $L^{g}$ is the gyrogeodesic given by

$$
L^{g}=\{b \oplus(t \otimes c): t \in \mathbb{R}\} .
$$

Proof. Let $b \in P_{G}^{\perp}$ and $0 \neq c \in P_{G}$. Since for any $t \in \mathbb{R}$,

$$
b \oplus(t \otimes c)=\phi_{1}(b, t \otimes c) b+\phi_{2}(b, t \otimes c)(t \otimes c),
$$

we know that $L^{g}$ is a gyroline in $G$ in the plane defined by the vectors $b$ and $c$. Now, we consider $a \in P_{G}$ arbitrary. Since it is always possible to find $t \in \mathbb{R}$ such that $\|t \otimes c\|=\|a\|$, then there exists an automorphism $\tau \in \operatorname{Aut}(G, \oplus, \otimes)$ such that $a=\tau(t \otimes c)$ and $\tau$ leaves $P_{G}$ invariant and takes each element of $P_{G}^{\perp}$ as a fixed point. This means that $\tau \in \operatorname{Aut}\left(P_{G}, \oplus, \otimes\right)$. In particular, since $b \in P_{G}^{\perp}$ then $\tau b=b$, and consequently, $\tau^{-1} b=b$. Then, by the invariance property P3 of gyrolines (described in Section 2) we have

$$
b \oplus a=b \oplus(\tau(t \otimes c))=\tau\left(\tau^{-1} b \oplus(t \otimes c)\right)=\tau(b \oplus(t \otimes c)) .
$$

Thus, we get $b \oplus P_{G}=\left\{\tau L^{g}: \tau \in \operatorname{Aut}\left(P_{G}, \oplus, \otimes\right)\right\}$. 
In an analogous way, we can characterize the right equivalence classes $P_{G} \oplus b$ with $b \in P_{G}^{\perp}$ in terms of the automorphisms of $\left(P_{G}, \oplus, \otimes\right)$ acting on a given cogyroline $L^{c}$.

Theorem 15. Let $b \in P_{G}^{\perp}$ and $0 \neq c \in P_{G}$ be fixed. Then

$$
P_{G} \oplus b=\left\{\tau L^{c}: \tau \in \operatorname{Aut}\left(P_{G}, \oplus, \otimes\right)\right\},
$$

where the cogyroline $L^{c}$ is the cogyrogeodesic given by

$$
L^{c}=\{(t \otimes c) \oplus b: t \in \mathbb{R}\} .
$$

Proof. Let $b \in P_{G}^{\perp}$ and $0 \neq c \in P_{G}$. Since for any $t \in \mathbb{R}$,

$$
(t \otimes c) \oplus b=\phi_{1}(t \otimes c, b)(t \otimes c)+\phi_{2}(t \otimes c, b) b,
$$

we know that $L^{c}$ is a cogyroline in $G$ in the plane defined by the vectors $b$ and $c$. Now, we consider $a \in P_{G}$ arbitrary. Then there exist $t \in \mathbb{R}$ and $\tau \in \operatorname{Aut}\left(P_{G}, \oplus, \otimes\right)$ such that $a=\tau(t \otimes c)$ and $\tau$ leaves $P_{G}$ invariant. Thus, by property P3 of gyrolines since $\tau^{-1} b=b$, we have

$$
a \oplus b=(\tau(t \otimes c)) \oplus b=\tau\left((t \otimes c) \oplus \tau^{-1} b\right)=\tau((t \otimes c) \oplus b) .
$$

Hence, we get $P_{G} \oplus b=\left\{\tau L^{c}: \tau \in \operatorname{Aut}\left(P_{G}, \oplus, \otimes\right)\right\}$.

\section{Fiber Bundles and Sections of Real Inner Product Gyrogroups}

We denote $(G, X, \pi, Y)$ as a fiber bundle with base space $X$, fiber $Y$, and bundle map $\pi: G \rightarrow X$. A global section of the fiber bundle $(G, X, \pi, Y)$ is a continuous map $f: X \rightarrow G$ such that $\pi(f(y))=y$ for all $y \in X$, while a local section is a map $f: U \rightarrow G$, where $U$ is an open set in $X$ and $\pi(f(x))=x$ for all $x \in U$.

According with the two unique decompositions $G=P_{G} \oplus P_{G}^{\perp}$ and $G=P_{G}^{\perp} \oplus P_{G}$, and using Lemma 1, Lemma 2, (24), and (28), we can define four different fiber bundle structures on $(G, \oplus)$ with fiber bundle mappings given by

$$
\begin{array}{rlrl}
\pi_{1}: P_{G} \oplus P_{\bar{G}}^{\perp} & \rightarrow\left(G / P_{G}, \sim_{r}\right) & \pi_{2}: P_{G}^{\perp} \oplus P_{G} & \rightarrow\left(G / P_{G}, \sim_{\ell}\right) \\
a \oplus b & \mapsto[a \oplus b]_{r}=[b]_{r}=P_{G} \oplus b & b \oplus a & \mapsto b \oplus]_{\ell}=[b]_{\ell}=b \oplus P_{G} \\
\pi_{3}: P_{G}^{\perp} \oplus P_{G} & \rightarrow\left(G / P_{G}, \sim_{r}\right) & & \\
b \oplus a & \mapsto[b \oplus a]_{r}=P_{G} \oplus \mu_{2}(b, a) b & a \oplus b & \mapsto\left(G / P_{G}, \sim_{\ell}\right) \\
b \quad P_{G} \perp & \rightarrow b]_{\ell}=\mu_{1}(a, b) b \oplus P_{G} .
\end{array}
$$

We remark that the mappings $\pi_{3}$ and $\pi_{4}$ defined in (38) correct the definitions presented in [5].

It is easy to see that the first and the second bundles are trivial ones. The first bundle $\pi_{1}$ is isomorphic to the trivial bundle $\left(P_{G} \times P_{G}^{\perp}, P_{G}^{\perp}, \mathcal{Q}^{r}, P_{G}\right)$, where $\mathcal{Q}^{r}$ is the projection onto the second factor defined by:

$$
\mathcal{Q}^{r}: P_{G} \oplus P_{G}^{\perp} \rightarrow P_{G}^{\perp}, \quad a \oplus b \mapsto b .
$$

Hence, the following diagram commutes:

$$
\begin{aligned}
& G=P_{G} \oplus P_{G}^{\perp} \stackrel{\pi_{1}}{\longrightarrow}\left(G / P_{G}, \sim_{r}\right) \\
& \downarrow \text { id } \quad \downarrow \Phi_{1} \\
& P_{G} \oplus P_{G}^{\perp} \stackrel{\mathcal{Q}^{r}}{\longrightarrow} P_{G}^{\perp}
\end{aligned}
$$


where $\Phi_{1}$ is the isomorphism between $\left(G / P_{G}, \sim_{r}\right)$ and $P_{G}^{\perp}$ given by $\Phi_{1}\left(P_{G} \oplus b\right)=b$, for any $b \in P_{G}^{\perp}$. All global sections of the first fiber bundle are given by

$$
f\left(P_{G} \oplus b\right)=g(b) \oplus b,
$$

for any continuous map $g: P_{G}^{\perp} \rightarrow P_{G}$. In fact, $\pi_{1}\left(f\left(P_{G} \oplus b\right)\right)=\pi_{1}(g(b) \oplus b)=P_{G} \oplus b$, for all $b \in P_{G}^{\perp}$.

The second bundle $\pi_{2}$ is isomorphic to the trivial bundle $\left(P_{G}^{\perp} \times P_{G}, P_{G}^{\perp}, \mathcal{Q}^{\ell}, P_{G}\right)$, where $\mathcal{Q}^{\ell}$ is the projection onto the first factor defined by:

$$
\mathcal{Q}^{\ell}: P_{G}^{\perp} \oplus P_{G} \rightarrow P_{G}^{\perp}, \quad b \oplus a \mapsto b .
$$

Indeed, the following diagram commutes

$$
\begin{aligned}
& G=P_{G}^{\perp} \oplus P_{G} \stackrel{\pi_{2}}{\longrightarrow}\left(G / P_{G}, \sim_{\ell}\right) \\
& \downarrow \text { id } \downarrow \Phi_{2} \\
& P_{G}^{\perp} \oplus P_{G} \stackrel{\mathcal{Q}^{\ell}}{\longrightarrow} P_{G}^{\perp}
\end{aligned}
$$

where $\Phi_{2}$ is the isomorphism between $\left(G / P_{G}, \sim_{\ell}\right)$ and $P_{G}^{\perp}$ given by $\Phi_{2}\left(b \oplus P_{G}\right)=b$, for any $b \in P_{G}^{\perp}$. All global sections of the second fiber bundle are given by

$$
f\left(b \oplus P_{G}\right)=b \oplus g(b),
$$

for any continuous map $g: P_{G}^{\perp} \rightarrow P_{G}$. Note that $\pi_{2}\left(f\left(b \oplus P_{G}\right)\right)=\pi_{2}(b \oplus g(b))=b \oplus P_{G}$, for all $b \in P_{G}^{\perp}$.

In the third and fourth bundles we will consider the sections obtained from the quotient spaces $\left(G / P_{G}^{\perp}, \sim_{r}\right)$ and $\left(G / P_{G}^{\perp}, \sim_{\ell}\right)$.

In the third case if we consider for any $a \in P_{G}$ fixed the map $\tau_{a}^{(1)}$ defined by

$$
\begin{aligned}
\tau_{a}^{(1)}:\left(G / P_{G}, \sim_{r}\right) & \rightarrow G \\
{[b]_{r} } & \mapsto a \oplus b
\end{aligned}
$$

with $b \in P_{G}^{\perp}$, which means that $\tau_{a}^{(1)}\left(G / P_{G}, \sim_{r}\right)=a \oplus P_{G}^{\perp}$ is a left coset in $\left(G / P_{G}^{\perp}, \sim_{\ell}\right)$, we obtain a global section. In fact, $\pi_{3}\left(\tau_{a}^{(1)}\left([b]_{r}\right)\right)=\pi_{3}(a \oplus b)=P_{G} \oplus b=[b]_{r}$, for any $b \in P_{G}^{\perp}$. This means that the left cosets of $\left(G / P_{G}^{\perp}, \sim_{\ell}\right)$ are global sections for $\left(G / P_{G}, \sim_{r}\right)$.

However, if we consider the map $\tau_{a}^{(2)}$ defined for any $a \in P_{G} \backslash\{0\}$ by

$$
\begin{aligned}
\tau_{a}^{(2)}:\left(G / P_{G}, \sim_{r}\right) & \rightarrow G \\
{[b]_{r} } & \mapsto b \oplus a
\end{aligned}
$$

for $b \in P_{G}^{\perp}$, which means that $\tau_{a}^{(2)}\left(G / P_{G}, \sim_{r}\right)=P_{G}^{\perp} \oplus a$ is a right coset in $\left(G / P_{G}^{\perp}, \sim_{r}\right)$, then by (38) we have

$$
\pi_{3}\left(\tau_{a}^{(2)}\left([b]_{r}\right)\right)=\pi_{3}(b \oplus a)=P_{G} \oplus \mu_{2}(b, a) b=\left[\mu_{2}(b, a) b\right]_{r}
$$

with $\mu_{2}(b, a)$ given by (27) with the order of $a$ and $b$ being changed. Depending on the properties of the mapping $\mu_{2}$ we can have global or local sections. If for $a \in P_{G}$ fixed we have $\left\{\mu_{2}(b, a) b: b \in P_{G}^{\perp}\right\}=P_{G}^{\perp}$ then $\tau_{a}^{(2)}$ is a global section while if $\left\{\mu_{2}(b, a) b: b \in P_{G}^{\perp}\right\} \subsetneq P_{G}^{\perp}$ then $\tau_{a}^{(2)}$ is only a local section for the fiber bundle defined by $\pi_{3}$. The case $a=0$ gives a global section since

$$
\pi_{3}\left(\tau_{0}^{(2)}\left([b]_{r}\right)\right)=\pi_{3}(b \oplus 0)=\pi_{3}(0 \oplus b)=[b]_{r}, \quad \text { for any } b \in P_{G}^{\perp} .
$$


The conclusions obtained for the sections $\tau_{a}^{(1)}$ and $\tau_{a}^{(2)}$ are valid for the fiber bundles associated to the projection mappings $\pi_{1}$ and $\pi_{3}$.

In the fourth case we consider the sections $\tau_{a}^{(3)}$ and $\tau_{a}^{(4)}$ defined for any $a \in P_{G}$ by

$$
\begin{aligned}
\tau_{a}^{(3)}:\left(G / P_{G}, \sim_{\ell}\right) & \rightarrow G \\
{[b]_{\ell} } & \mapsto b \oplus a
\end{aligned}
$$

and

$$
\begin{aligned}
\tau_{a}^{(4)}:\left(G / P_{G}, \sim_{\ell}\right) & \rightarrow G \\
{[b]_{\ell} } & \mapsto a \oplus b .
\end{aligned}
$$

It is easy to see that $\tau_{a}^{(3)}$ is a global section for $\pi_{4}$ since

$$
\pi_{4}\left(\tau_{a}^{(3)}\left([b]_{\ell}\right)\right)=\pi_{4}(b \oplus a)=b \oplus P_{G}=[b]_{\ell}
$$

for any $b \in P_{G}^{\perp}$. Since $\tau_{a}^{(3)}\left(G / P_{G}, \sim_{\ell}\right)=P_{G}^{\perp} \oplus a$ is a right coset of $\left(G / P_{G}^{\perp}, \sim_{r}\right)$ then the right cosets of $\left(G / P_{G}^{\perp}, \sim_{r}\right)$ are global sections for $\left(G / P_{G}, \sim_{\ell}\right)$.

Concerning $\tau_{a}^{(4)}$ we have

$$
\pi_{4}\left(\tau_{a}^{(4)}\left([b]_{\ell}\right)\right)=\pi_{4}(a \oplus b)=\mu_{1}(a, b) b \oplus P_{G}=\left[\mu_{1}(a, b) b\right]_{\ell}
$$

for any $a \in P_{G}$, where $\mu_{1}(a, b)$ is given by (27). If for $a \in P_{G}$ fixed we have $\left\{\mu_{1}(a, b) b: b \in P_{G}^{\perp}\right\}=P_{G}^{\perp}$ then $\tau_{a}^{(4)}$ is a global section while if $\left\{\mu_{1}(a, b) b: b \in P_{G}^{\perp}\right\} \subsetneq P_{G}^{\perp}$ then $\tau_{a}^{(4)}$ is only a local section for the fiber bundle $\pi_{4}$. The case $a=0$ gives a global section since

$$
\pi_{4}\left(\tau_{0}^{(4)}\left([b]_{\ell}\right)\right)=\pi_{4}(0 \oplus b)=\pi_{3}(b \oplus 0)=b \oplus P_{G}=[b]_{\ell}
$$

for any $b \in P_{G}^{\perp}$. The conclusions obtained for the sections $\tau_{a}^{(3)}$ and $\tau_{a}^{(4)}$ are valid for the fiber bundles associated to the projection mappings $\pi_{2}$ and $\pi_{4}$.

We summarize in the next theorem the duality relations between left and right cosets obtained from the orthogonal factorization of $G$ by $P_{G}$ and by $P_{G}^{\perp}$.

Theorem 16. Let $P_{G}$ be a subgyrogroup of $(G, \oplus)$. The following relations hold:

1. The cosets of $\left(G / P_{G}^{\perp}, \sim_{\ell}\right)$ are global sections for the quotient space $\left(G / P_{G}, \sim_{r}\right)$.

2. The cosets of $\left(G / P_{G}^{\perp}, \sim_{r}\right)$ are global sections for the quotient space $\left(G / P_{G}, \sim_{r}\right)$ if $\left\{\mu_{2}(b, a) b\right.$ : $\left.b \in P_{G}^{\perp}\right\}=P_{G}^{\perp}$ or local sections if $\left\{\mu_{2}(b, a) b: b \in P_{G}^{\perp}\right\} \subsetneq P_{G}^{\perp}$.

3. The cosets of $\left(G / P_{G}^{\perp}, \sim_{r}\right)$ are global sections for the quotient space $\left(G / P_{G}, \sim_{\ell}\right)$.

4. The cosets of $\left(G / P_{G}^{\perp}, \sim_{\ell}\right)$ are global sections for the quotient space $\left(G / P_{G}, \sim_{\ell}\right)$ if $\left\{\mu_{1}(a, b) b\right.$ : $\left.b \in P_{G}^{\perp}\right\}=P_{G}^{\perp}$ or local sections if $\left\{\mu_{1}(a, b) b: b \in P_{G}^{\perp}\right\} \subsetneq P_{G}^{\perp}$.

\section{Examples of Real Inner Product Gyrogroups}

In this section, we show that four standard gyrogroups known in the literature are indeed real inner product gyrogroups that admit orthogonal decompositions.

\subsection{Euclidean Einstein Gyrogroup}

The (Euclidean) Einstein gyrogroup [10] consists of the open unit ball in $\mathbb{R}^{n}$,

$$
\mathbb{B}=\left\{\mathbf{v} \in \mathbb{R}^{n}:\|\mathbf{v}\|<1\right\},
$$


endowed with Einstein addition $\oplus_{E}$ defined by

$$
\mathbf{u} \oplus_{E} \mathbf{v}=\frac{1}{1+\langle\mathbf{u}, \mathbf{v}\rangle}\left(\mathbf{u}+\frac{1}{\gamma_{\mathbf{u}}} \mathbf{v}+\frac{\gamma_{\mathbf{u}}}{1+\gamma_{\mathbf{u}}}\langle\mathbf{u}, \mathbf{v}\rangle \mathbf{u}\right),
$$

for all $\mathbf{u}, \mathbf{v} \in \mathbb{B}$, where $\langle$,$\rangle denotes the inner product in \mathbb{R}^{n}$ and $\gamma_{\mathbf{u}}$ is the Lorentz factor given by $\gamma_{\mathbf{u}}=\frac{1}{\sqrt{1-\|\mathbf{u}\|^{2}}}$. Einstein addition satisfies the gamma identity

$$
\gamma_{\mathbf{u} \oplus_{E} \mathbf{v}}=\gamma_{\mathbf{u}} \gamma_{\mathbf{v}}(1+\langle\mathbf{u}, \mathbf{v}\rangle)
$$

In view of (46), we define

$$
\phi_{1}(\mathbf{u}, \mathbf{v})=\frac{1+\gamma_{\mathbf{u}}+\gamma_{\mathbf{u}}\langle\mathbf{u}, \mathbf{v}\rangle}{(1+\langle\mathbf{u}, \mathbf{v}\rangle)\left(1+\gamma_{\mathbf{u}}\right)} \quad \text { and } \quad \phi_{2}(\mathbf{u}, \mathbf{v})=\frac{1}{\gamma_{\mathbf{u}}(1+\langle\mathbf{u}, \mathbf{v}\rangle)}
$$

for all $\mathbf{u}, \mathbf{v} \in \mathbb{B}$. The gyrogroup identity of $\left(\mathbb{B}, \oplus_{E}\right)$ is the zero vector $\mathbf{0}$. Further, $\ominus_{E} \mathbf{u}=-\mathbf{u}$ for all $\mathbf{u} \in \mathbb{B}$. Let $\mathbf{u}, \mathbf{v} \in \mathbb{B}$. By the Cauchy-Schwarz inequality,

$$
-1<-\|\mathbf{u}\|\|\mathbf{v}\| \leq\langle\mathbf{u}, \mathbf{v}\rangle \leq\|\mathbf{u}\|\|\mathbf{v}\|<1 .
$$

Hence, $1+\langle\mathbf{u}, \mathbf{v}\rangle>0$. This implies that $\phi_{1}(\mathbf{u}, \mathbf{v})>0$ and $\phi_{2}(\mathbf{u}, \mathbf{v})>0$.

Clearly, the Einstein gyrogroup satisfies (H1). Suppose that $\mathbf{u}, \mathbf{v} \in \mathbb{B}, \mathbf{u}+\mathbf{v} \in \mathbb{B}$, and $\langle\mathbf{u}, \mathbf{v}\rangle=0$. Then by (47) it is easy to see that the unique solution of the system of equations

$$
\left\{\begin{array}{l}
\phi_{1}\left(\lambda_{1} \mathbf{u}, \lambda_{2} \mathbf{v}\right) \lambda_{1} \mathbf{u}=\mathbf{u} \\
\phi_{2}\left(\lambda_{1} \mathbf{u}, \lambda_{2} \mathbf{v}\right) \lambda_{2} \mathbf{v}=\mathbf{v}
\end{array}\right.
$$

is given by $\lambda_{1}=\lambda_{1}(u, v)=1$ and $\lambda_{2}=\lambda_{2}(u, v)=\gamma_{\mathbf{u}}$. Thus, $\left(x=\mathbf{u}, y=\gamma_{\mathbf{u}} \mathbf{v}\right)$ is the solution to the system (16), where $c_{1}=\mathbf{u}$ and $c_{2}=\mathbf{v}$. We claim that $y \in \mathbb{B}$. To prove the claim, suppose to the contrary that $y \notin \mathbb{B}$. Hence, $\left\|\gamma_{\mathbf{u}} \mathbf{v}\right\| \geq 1$. This implies $\gamma_{\mathbf{u}}\|\mathbf{v}\| \geq 1$ and so $\frac{\|\mathbf{v}\|^{2}}{1-\|\mathbf{u}\|^{2}} \geq 1$. Hence, $\|\mathbf{u}+\mathbf{v}\|^{2}=\|\mathbf{u}\|^{2}+\|\mathbf{v}\|^{2} \geq 1$, contrary to the assumption that $\mathbf{u}+\mathbf{v} \in \mathbb{B}$. Thus, $y \in \mathbb{B}$. Hence, the Einstein gyrogroup satisfies (H2).

Now, let $P$ be a linear subspace of $\mathbb{R}^{n}, P_{\mathbb{B}}=P \cap \mathbb{B}$, and $P_{\mathbb{B}}^{\perp}=P^{\perp} \cap \mathbb{B}$. Then $\mathbb{R}^{n}=P \oplus P^{\perp}$ and $P_{\mathbb{B}}$ and $P_{\mathbb{B}}^{\perp}$ are subgyrogroups of the Einstein gyrogroup $\left(\mathbb{B}, \oplus_{E}\right)$. The next three theorems are consequences of Theorems 5, 8, 10,13 obtained in Section 3.

Theorem 17. Let $P_{\mathbb{B}}$ be a subgyrogroup of $\left(\mathbb{B}, \oplus_{E}\right)$. For all $\mathbf{w} \in \mathbb{B}$ such that $\mathbf{w}=\mathbf{u}+\mathbf{v}$, with $\mathbf{u} \in P_{\mathbb{B}}$ and $\mathbf{v} \in P_{\mathbb{B}}^{\perp}$, the unique orthogonal decompositions of $\mathbf{w}$ according to $\oplus_{E}$ are given by

$$
\mathbf{w}=\lambda_{1}(\mathbf{u}, \mathbf{v}) \mathbf{u} \oplus_{E} \lambda_{2}(\mathbf{u}, \mathbf{v}) \mathbf{v} \text { and } \mathbf{w}=\lambda_{3}(\mathbf{u}, \mathbf{v}) \mathbf{v} \oplus_{E} \lambda_{4}(\mathbf{u}, \mathbf{v}) \mathbf{u},
$$

where

$$
\begin{array}{lll}
\lambda_{1}(\mathbf{u}, \mathbf{v})=1 & \text { and } & \lambda_{2}(\mathbf{u}, \mathbf{v})=\gamma_{\mathbf{u}} \\
\lambda_{3}(\mathbf{u}, \mathbf{v})=1 & \text { and } & \lambda_{4}(\mathbf{u}, \mathbf{v})=\lambda_{2}(\mathbf{v}, \mathbf{u})=\gamma_{\mathbf{v}}
\end{array}
$$


As a consequence $\mathbb{B}=P_{\mathbb{B}} \oplus_{E} P_{\mathbb{B}}^{\perp}$ and $\mathbb{B}=P_{\mathbb{B}}^{\perp} \oplus_{E} P_{\mathbb{B}}$. By (20) the orthogonal gyroprojectors of the Einstein gyrogroup $\left(\mathbb{B}, \oplus_{E}\right)$ onto the subgyrogroups $P_{\mathbb{B}}$ and $P_{\mathbb{B}}^{\perp}$ are given by

$$
\begin{array}{ll}
\mathcal{P}_{E}^{\ell}: \mathbb{B}=P_{\mathbb{B}} \oplus_{E} P_{\mathbb{B}}^{\perp} \rightarrow P_{\mathbb{B}}, & \mathcal{P}_{E}^{\ell}(\mathbf{w})=\mathbf{u}, \\
\mathcal{Q}_{E}^{r}: \mathbb{B}=P_{\mathbb{B}} \oplus_{E} P_{\mathbb{B}}^{\perp} \rightarrow P_{\mathbb{B}}^{\perp}, & \mathcal{Q}_{E}^{r}(\mathbf{w})=\gamma_{\mathbf{u}} \mathbf{v}, \\
\mathcal{Q}_{E}^{\ell}: \mathbb{B}=P_{\mathbb{B}}^{\perp} \oplus_{E} P_{\mathbb{B}} \rightarrow P_{\mathbb{B}}^{\perp}, & \mathcal{Q}_{E}^{\ell}(\mathbf{w})=\mathbf{v}, \\
\mathcal{P}_{E}^{r}: \mathbb{B}=P_{\mathbb{B}}^{\perp} \oplus_{E} P_{\mathbb{B}} \rightarrow P_{\mathbb{B}}, & \mathcal{P}_{E}^{r}(\mathbf{w})=\gamma_{\mathbf{v}} \mathbf{u} .
\end{array}
$$

Theorem 18. Let $P_{\mathbb{B}}$ be a subgyrogroup of $\left(\mathbb{B}, \oplus_{E}\right)$. Then the sets $\left\{b \oplus_{E} P_{\mathbb{B}}: b \in P_{\mathbb{B}}^{\perp}\right\}$ and $\left\{P_{\mathbb{B}} \oplus_{E} b: b \in P_{\mathbb{B}}^{\perp}\right\}$ are disjoint partitions of $\mathbb{B}$, that is,

$$
\mathbb{B}=\bigcup_{b \in P_{\mathbb{B}}^{\perp}}\left(b \oplus_{E} P_{\mathbb{B}}\right) \text { and } \mathbb{B}=\bigcup_{b \in P_{\mathbb{B}}^{\perp}}\left(P_{\mathbb{B}} \oplus_{E} b\right)
$$

Moreover, $\left(\mathbb{B} / P_{\mathbb{B}}, \sim_{\ell}\right)=\left\{b \oplus_{E} P_{\mathbb{B}}: b \in P_{\mathbb{B}}^{\perp}\right\}$ and $\left(\mathbb{B} / P_{\mathbb{B}}, \sim_{r}\right)=\left\{P_{\mathbb{B}} \oplus_{E} b: b \in P_{\mathbb{B}}^{\perp}\right\}$.

Theorem 19. Let $P_{\mathbb{B}}$ be a subgyrogroup of $\left(\mathbb{B}, \oplus_{E}\right)$. Then the following are gyrogroup isomorphisms:

$$
\left(\mathbb{B} / P_{\mathbb{B}}, \sim_{\ell}\right) \cong\left(P_{\mathbb{B}}^{\perp}, \oplus_{E}\right) \cong\left(\mathbb{B} / P_{\mathbb{B}}, \sim_{r}\right) .
$$

In order to see a remarkable connection between gyroprojections and Euclidean projections, let us refer to a version of Einstein addition defined on an open ball of $\mathbb{R}^{n}$ of radius $t$, where $t$ is a positive number. Set

$$
\mathbb{B}_{t}=\left\{\mathbf{v} \in \mathbb{R}^{n}:\|\mathbf{v}\|<t\right\} .
$$

Recall that the $t$-Einstein addition is defined by

$$
\mathbf{u} \oplus_{E_{t}} \mathbf{v}=\frac{1}{1+\frac{\langle\mathbf{u}, \mathbf{v}\rangle}{t^{2}}}\left(\mathbf{u}+\frac{1}{\gamma_{\mathbf{u}}} \mathbf{v}+\frac{1}{t^{2}} \frac{\gamma_{\mathbf{u}}}{1+\gamma_{\mathbf{u}}}\langle\mathbf{u}, \mathbf{v}\rangle \mathbf{u}\right)
$$

for all $\mathbf{u}, \mathbf{v} \in \mathbb{B}_{t}$ and $\gamma_{\mathbf{u}}$ is redefined by $\gamma_{\mathbf{u}}=\left(1-\frac{\|\mathbf{u}\|^{2}}{t^{2}}\right)^{-\frac{1}{2}}$. When $t$ is arbitrarily large (that is, $t \rightarrow+\infty), \mathbb{B}_{t}$ expands to the whole space $\mathbb{R}^{n}, \oplus_{E_{t}}$ reduces to ordinary vector addition of $\mathbb{R}^{n}$, and $\gamma_{\mathbf{u}} \rightarrow 1$. Therefore, the orthogonal gyroprojections of the $t$-Einstein gyrogroup reduce to ordinary projections of $\mathbb{R}^{n}$ :

$$
\mathcal{P}(\mathbf{u}+\mathbf{v})=\mathbf{u} \quad \text { and } \quad \mathcal{Q}(\mathbf{u}+\mathbf{v})=\mathbf{v},
$$

for all $\mathbf{u} \in P$ and $\mathbf{v} \in P^{\perp}$.

Finally, we analyze the fiber bundles and sections of the Einstein gyrogroup arisen from the two orthogonal decompositions $\mathbb{B}=P_{\mathbb{B}} \oplus_{E} P_{\mathbb{B}}^{\perp}$ and $\mathbb{B}=P_{\mathbb{B}}^{\perp} \oplus_{E} P_{\mathbb{B}}$. First we give the relation between these two decompositions according to Theorem 6.

Theorem 20. Let $\mathbf{u}, \mathbf{v} \in\left(\mathbb{B}, \oplus_{E}\right)$ such that $\langle\mathbf{u}, \mathbf{v}\rangle=0$. Then

$$
\mathbf{u} \oplus_{E} \mathbf{v}=\mu_{1}(\mathbf{u}, \mathbf{v}) \mathbf{v} \oplus_{E} \mu_{2}(\mathbf{u}, \mathbf{v}) \mathbf{u},
$$

where

$$
\mu_{1}(\mathbf{u}, \mathbf{v})=\frac{1}{\gamma_{\mathbf{u}}} \quad \text { and } \quad \mu_{2}(\mathbf{u}, \mathbf{v})=\gamma_{\frac{1}{\gamma \mathbf{u}}} \mathbf{v}
$$


Proof. By (27), (47), (49), we have

$$
\begin{aligned}
& \mu_{1}(\mathbf{u}, \mathbf{v})=\lambda_{3}\left(\phi_{1}(\mathbf{u}, \mathbf{v}) \mathbf{u}, \phi_{2}(\mathbf{u}, \mathbf{v}) \mathbf{v}\right) \phi_{2}(\mathbf{u}, \mathbf{v})=\frac{1}{\gamma_{\mathbf{u}}} \\
& \mu_{2}(\mathbf{u}, \mathbf{v})=\lambda_{4}\left(\phi_{1}(\mathbf{u}, \mathbf{v}) \mathbf{u}, \phi_{2}(\mathbf{u}, \mathbf{v}) \mathbf{v}\right) \phi_{1}(\mathbf{u}, \mathbf{v})=\lambda_{4}\left(\mathbf{u}, \frac{1}{\gamma_{\mathbf{u}}} \mathbf{v}\right)=\gamma_{\frac{1}{\gamma \mathbf{u}}} \mathbf{v}
\end{aligned}
$$

Now, we analyze the sections $\tau_{\mathbf{u}}^{(2)}$ and $\tau_{\mathbf{u}}^{(4)}$ defined by (40) and (43), respectively, with the changes $a \leftrightarrow \mathbf{u} \in P_{\mathbb{B}}$ and $b \leftrightarrow \mathbf{v} \in P_{\mathbb{B}}^{\perp}$.

Since for any $\mathbf{u} \in P_{\mathbb{B}} \backslash\{\mathbf{0}\}$ it turns out that $\mu_{1}(\mathbf{u}, \mathbf{v})\|\mathbf{v}\|=\sqrt{1-\|\mathbf{u}\|^{2}}\|\mathbf{v}\|$ is a strictly increasing function such that $\mu_{1}(\mathbf{u}, \mathbf{v})\|\mathbf{v}\| \in\left[0, \sqrt{1-\|\mathbf{u}\|^{2}}\left[\subsetneq\left[0,1\left[\right.\right.\right.\right.$ for all $\mathbf{v} \in P_{\mathbb{B}}^{\perp}$, we conclude that $\left\{\mu_{1}(\mathbf{u}, \mathbf{v}) \mathbf{v}\right.$ : $\left.\mathbf{v} \in P_{\mathbb{B}}^{\perp}\right\} \subsetneq P_{\mathbb{B}}^{\perp}$. Then for any $\mathbf{u} \in P_{\mathbb{B}} \backslash\{\mathbf{0}\}$, the section $\tau_{\mathbf{u}}^{(2)}$ is a local section for the fiber bundles $\pi_{1}$ and $\pi_{3}$ defined in (38). In the case when $\mathbf{u}=\mathbf{0}$ the section $\tau_{\mathbf{0}}^{(2)}$ is a global section as seen in (41).

Concerning the section $\tau_{\mathbf{u}}^{(4)}$, since for each $\mathbf{u} \in P_{\mathbb{B}}$ we have that $\mu_{2}(\mathbf{v}, \mathbf{u})\|\mathbf{v}\|=\frac{\|\mathbf{v}\|}{\sqrt{1-\left(1-\|\mathbf{v}\|^{2}\right)\|\mathbf{u}\|^{2}}}$ is a strictly increasing function in the variable $\|\mathbf{v}\|$ such that $\mu_{2}(\mathbf{0}, \mathbf{u})\|\mathbf{0}\|=0$ and $\lim _{\|\mathbf{v}\| \rightarrow 1} \mu_{2}(\mathbf{v}, \mathbf{u})\|\mathbf{v}\|=1$, we conclude that $\left\{\mu_{2}(\mathbf{v}, \mathbf{u}) \mathbf{v}: \mathbf{v} \in P_{\mathbb{B}}^{\perp}\right\}=P_{\mathbb{B}}^{\perp}$. Hence, for any $\mathbf{u} \in P_{\mathbb{B}}$, it follows that $\tau_{\mathbf{u}}^{(4)}$ is a global section for the fiber bundles $\pi_{2}$ and $\pi_{4}$ defined in (38).

In Figure 1 we show the plots of $\mu_{1}(\mathbf{u}, \mathbf{v})\|\mathbf{v}\|$ and $\mu_{2}(\mathbf{v}, \mathbf{u})\|\mathbf{v}\|$, with $\|\mathbf{u}\|,\|\mathbf{v}\|<1$, that explain the different behavior between local and global sections.

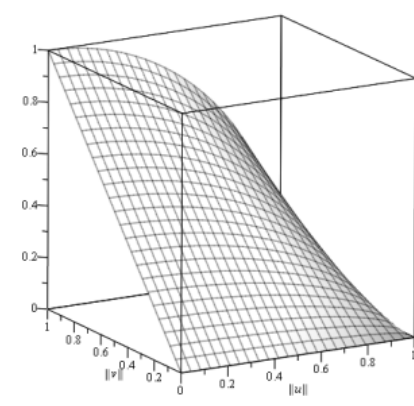

(a)

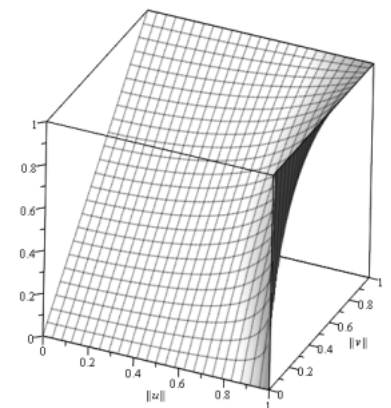

(b)

Figure 1. (a) Plot of $\mu_{1}(\mathbf{u}, \mathbf{v})\|\mathbf{v}\|$ with $\|\mathbf{u}\|,\|\mathbf{v}\|<1$; (b) plot of $\mu_{2}(\mathbf{v}, \mathbf{u})\|\mathbf{v}\|$ with $\|\mathbf{u}\|,\|\mathbf{v}\|<1$, for the Einstein gyrogroup.

By the results obtained in Section 4 and the previous conclusions we summarize in the next theorem the duality relations between left and right cosets obtained from the orthogonal factorization of $\mathbb{B}$ by $P_{\mathbb{B}}$ and by $P_{\mathbb{B}}^{\perp}$, for the Einstein gyrogroup.

Theorem 21. Let $P_{\mathbb{B}}$ be a subgyrogroup of $\left(\mathbb{B}, \oplus_{E}\right)$. The following duality relations hold:

1. The cosets of $\left(\mathbb{B} / P_{\mathbb{B}}^{\perp}, \sim_{r}\right)$ are global sections for the quotient spaces $\left(\mathbb{B} / P_{\mathbb{B}}, \sim_{\ell}\right)$ and $\left(\mathbb{B} / P_{\mathbb{B}}, \sim_{r}\right)$, and vice versa.

2. The cosets of $\left(\mathbb{B} / P_{\mathbb{B}}^{\perp}, \sim_{\ell}\right)$ are global sections for the quotient space $\left(\mathbb{B} / P_{\mathbb{B}}, \sim_{r}\right)$, and vice versa.

3. The cosets of $\left(\mathbb{B} / P_{\mathbb{B}}^{\perp}, \sim_{\ell}\right)$ are local sections for the quotient space $\left(\mathbb{B} / P_{\mathbb{B}}, \sim_{\ell}\right)$ except the identity coset $\mathbf{0} \oplus_{E} P_{B}^{\perp}=P_{B}^{\perp}$ that is a global section, and vice versa.

To visualize left and right cosets we restrict now to the 3-dimensional space $\mathbb{R}^{3}$. In $\mathbb{R}^{3}$ the nontrivial linear subspaces are of dimension 1 (straight lines passing through the origin) or dimension 2 (planes passing through the origin). Let $L_{e_{3}}=\left\{\left(0,0, x_{3}\right): x_{3} \in \mathbb{R}\right\}$ be the straight line that passes through the origin and the North Pole $e_{3}=(0,0,1)$ and let $D_{e_{3}}=\left\{\left(x_{1}, x_{2}, 0\right): x_{1}, x_{2} \in \mathbb{R}\right\}$ be the plane that passes through the origin and is perpendicular to $e_{3}$. The restriction of $L_{e_{3}}$ and $D_{e_{3}}$ to $\mathbb{B}^{3}=\left\{x \in \mathbb{R}^{3}:\|x\|<1\right\}$ will be denoted by $L_{e_{3}}^{\mathbb{B}^{3}}$ and $D_{e_{3}}^{\mathbb{B}^{3}}$, respectively. Then $L_{e_{3}}^{\mathbb{B}^{3}}$ and $D_{e_{3}}^{\mathbb{B}^{3}}$ are subgyrogroups of $\left(\mathbb{B}^{3}, \oplus_{E}\right)$ and 
provide orthogonal gyrodecompositions of $\left(\mathbb{B}^{3}, \oplus_{E}\right)$. In Figures 2 and 3 we show the left and right cosets obtained from the orthogonal decomposition of $\mathbb{B}^{3}$ by $L_{e_{3}}^{\mathbb{B}^{3}}$ and $D_{e_{3}}^{\mathbb{B}^{3}}$.

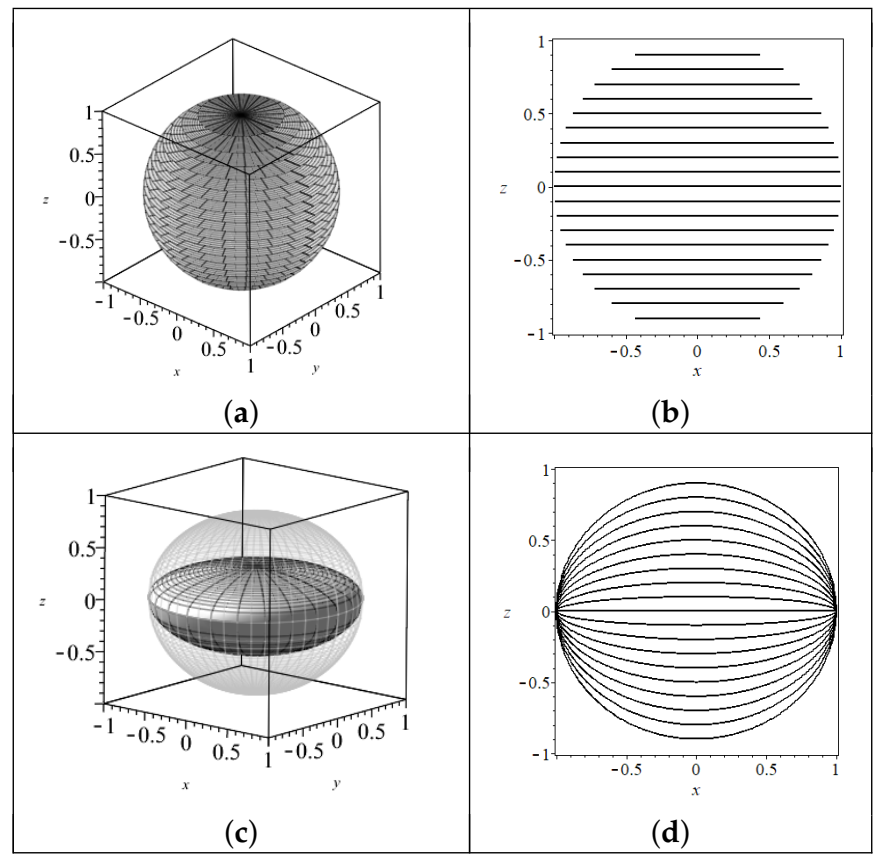

Figure 2. (a) Left cosets $\left(\mathbb{B}^{3} / D_{\mathfrak{e}_{3}}^{\mathbb{B}^{3}}, \sim_{\ell}\right)=\left\{\mathbf{v} \oplus_{E} D_{e_{3}}^{\mathbb{B}^{3}}, \mathbf{v} \in L_{e_{3}}^{\mathbb{B}^{3}}\right\} ;(\mathbf{b})$ gyrolines in the Einstein gyrovector plane (projection in the $x z$-plane of left cosets); (c) right cosets $\left(\mathbb{B}^{3} / D_{e_{3}}^{\mathbb{B}^{3}}, \sim_{r}\right)=\left\{D_{e_{3}}^{\mathbb{B}^{3}} \oplus_{E} \mathbf{u}, \mathbf{u} \in L_{e_{3}}^{\mathbb{B}^{3}}\right\}$; (d) Cogyrolines in the Einstein gyrovector plane (projection in the $x z$-plane of right cosets).

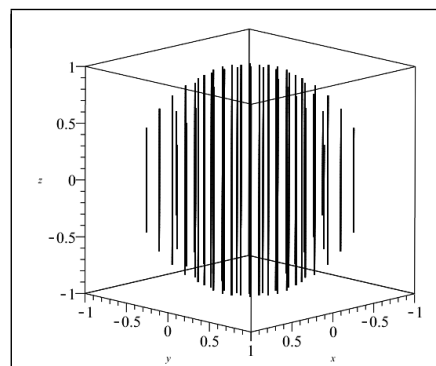

(a)

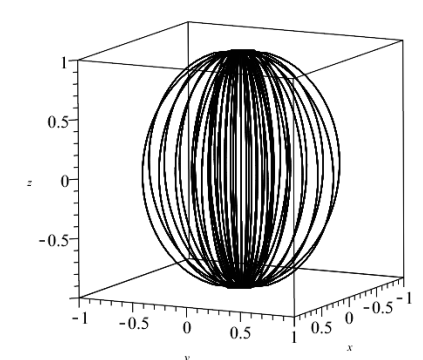

(c)

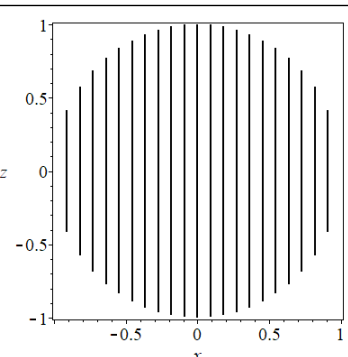

(b)

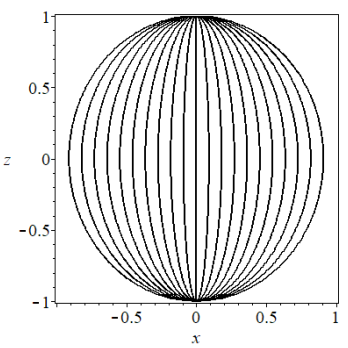

(d)

Figure 3. (a) Left cosets $\left(\mathbb{B}^{3} / L_{\mathbb{P}_{3}}^{\mathbb{B}^{3}}, \sim_{\ell}\right)=\left\{\mathbf{v} \oplus_{E} L_{\mathcal{B}_{3}}^{\mathbb{B}^{3}}, \mathbf{v} \in D_{\mathfrak{B}_{3}}^{\mathbb{B}^{3}}\right\} ;$ (b) Gyrolines in the Einstein gyrovector plane (projection in the $x z$-plane of left cosets); (c) Right cosets $\left(\mathbb{B}^{3} / L_{e_{3}}^{\mathbb{B}^{3}}, \sim_{r}\right)=\left\{L_{e_{3}}^{\mathbb{B}^{3}} \oplus_{E} \mathbf{v}, \mathbf{v} \in D_{e_{3}}^{\mathbb{B}^{3}}\right\}$; (d) Cogyrolines in the Einstein gyrovector plane (projection in the $x z$-plane of right cosets).

For higher dimensions $(n>3)$ we can have cosets with higher codimensions, which are surfaces of revolution obtained from the action of the automorphism group of $\left(P_{\mathbb{B}}, \oplus, \otimes\right)$ on the gyrolines and cogyrolines shown in Figures 2 and 3. Thus, all the information of these surfaces is encoded in the projection on an adequate plane. 


\subsection{Euclidean Möbius Gyrogroup}

The (Euclidean) Möbius gyrogroup [11] consists of the open unit ball $\mathbb{B}$ in $\mathbb{R}^{n}$ endowed with Möbius addition $\oplus_{M}$ defined by

$$
\mathbf{u} \oplus_{M} \mathbf{v}=\frac{\left(1+2\langle\mathbf{u}, \mathbf{v}\rangle+\|\mathbf{v}\|^{2}\right) \mathbf{u}+\left(1-\|\mathbf{u}\|^{2}\right) \mathbf{v}}{1+2\langle\mathbf{u}, \mathbf{v}\rangle+\|\mathbf{u}\|^{2}\|\mathbf{v}\|^{2}}
$$

for all $\mathbf{u}, \mathbf{v} \in \mathbb{B}$. The gyrogroup identity of $\left(\mathbb{B}, \oplus_{M}\right)$ is the zero vector $\mathbf{0}$, and $\ominus_{M} \mathbf{u}=-\mathbf{u}$ for all $\mathbf{u} \in \mathbb{B}$. A generalization of the Möius addition into the ball of any real inner product space was obtained in [12]. Möbius addition satisfies the gamma identity

$$
\gamma_{\mathbf{u} \oplus_{M} \mathbf{v}}=\gamma_{\mathbf{u}} \gamma_{\mathbf{v}} \sqrt{1+\langle\mathbf{u}, \mathbf{v}\rangle+\|\mathbf{u}\|^{2}\|\mathbf{v}\|^{2}}
$$

In view of (55), we define

$$
\phi_{1}(\mathbf{u}, \mathbf{v})=\frac{1+2\langle\mathbf{u}, \mathbf{v}\rangle+\|\mathbf{v}\|^{2}}{1+2\langle\mathbf{u}, \mathbf{v}\rangle+\|\mathbf{u}\|^{2}\|\mathbf{v}\|^{2}} \quad \text { and } \quad \phi_{2}(\mathbf{u}, \mathbf{v})=\frac{1-\|\mathbf{u}\|^{2}}{1+2\langle\mathbf{u}, \mathbf{v}\rangle+\|\mathbf{u}\|^{2}\|\mathbf{v}\|^{2}}
$$

for all $\mathbf{u}, \mathbf{v} \in \mathbb{B}$. Let $\mathbf{u}, \mathbf{v} \in \mathbb{B}$. Using the Cauchy-Schwarz inequality, we have

$$
1+2\langle\mathbf{u}, \mathbf{v}\rangle+\|\mathbf{v}\|^{2} \geq 1-2\|\mathbf{u}\|\|\mathbf{v}\|+\|\mathbf{v}\|^{2}=(\|\mathbf{v}\|-\|\mathbf{u}\|)^{2}+\left(1-\|\mathbf{u}\|^{2}\right) \geq 1-\|\mathbf{u}\|^{2}>0 .
$$

Hence, $\phi_{1}(\mathbf{u}, \mathbf{v})>0$. It is clear that $\phi_{2}(\mathbf{u}, \mathbf{v})>0$.

Clearly, the Möbius gyrogroup satisfies (H1). Suppose that $\mathbf{u}, \mathbf{v} \in \mathbb{B}, \mathbf{u}+\mathbf{v} \in \mathbb{B}$, and $\langle\mathbf{u}, \mathbf{v}\rangle=0$. In the case when $\mathbf{v}=\mathbf{0}$, set $\lambda_{1}=1$ and $\lambda_{2}=\frac{1}{1-\|\mathbf{u}\|^{2}}$. In the case when $\mathbf{v} \neq \mathbf{0}$ and $\mathbf{u}=\mathbf{0}$, set $\lambda_{1}=\frac{1}{1+\|\mathbf{v}\|^{2}}$ and $\lambda_{2}=1$. In both cases, direct computations show that

$$
\phi_{1}\left(\lambda_{1} \mathbf{u}, \lambda_{2} \mathbf{v}\right) \lambda_{1} \mathbf{u}=\mathbf{u} \quad \text { and } \quad \phi_{2}\left(\lambda_{1} \mathbf{u}, \lambda_{2} \mathbf{v}\right) \lambda_{2} \mathbf{v}=\mathbf{v} .
$$

Further, $\left(\lambda_{1} \mathbf{u}, \lambda_{2} \mathbf{v}\right) \in \mathbb{B} \times \mathbb{B}$. Therefore, we may assume that $\mathbf{u} \neq \mathbf{0}$ and $\mathbf{v} \neq \mathbf{0}$. Set

$$
\lambda_{1}=\frac{2}{1+\|\mathbf{u}\|^{2}+\|\mathbf{v}\|^{2}+\sqrt{\left(1-\left(\|\mathbf{u}\|^{2}+\|\mathbf{v}\|^{2}\right)\right)^{2}+4\|\mathbf{v}\|^{2}}}
$$

and

$$
\lambda_{2}=\frac{2}{1-\left(\|\mathbf{u}\|^{2}+\|\mathbf{v}\|^{2}\right)+\sqrt{\left(1-\left(\|\mathbf{u}\|^{2}+\|\mathbf{v}\|^{2}\right)\right)^{2}+4\|\mathbf{v}\|^{2}}} .
$$

An elaborate computation (see [7] for details) shows that $\lambda_{1}$ and $\lambda_{2}$ satisfy the following equalities:

$$
\frac{\lambda_{1}\left(1+\lambda_{2}^{2}\|\mathbf{v}\|^{2}\right)}{1+\lambda_{1}^{2} \lambda_{2}^{2}\|\mathbf{u}\|^{2}\|\mathbf{v}\|^{2}}=1 \quad \text { and } \quad \frac{\lambda_{2}\left(1-\lambda_{1}^{2}\|\mathbf{u}\|^{2}\right)}{1+\lambda_{1}^{2} \lambda_{2}^{2}\|\mathbf{u}\|^{2}\|\mathbf{v}\|^{2}}=1
$$

and that $\lambda_{1} \mathbf{u}, \lambda_{2} \mathbf{v} \in \mathbb{B}$. Hence, $\left(x=\lambda_{1} \mathbf{u}, y=\lambda_{2} \mathbf{v}\right) \in \mathbb{B} \times \mathbb{B}$ is a solution of the system of equations:

$$
\left\{\begin{array}{l}
\phi_{1}(x, y) x=\mathbf{u} \\
\phi_{2}(x, y) y=\mathbf{v}
\end{array} .\right.
$$

Next, we prove that the solution is unique. Suppose that $\left(\mathbf{x}^{\prime}, \mathbf{y}^{\prime}\right) \in \mathbb{B} \times \mathbb{B}$ is also a solution. Hence, $\phi_{1}\left(\mathbf{x}^{\prime}, \mathbf{y}^{\prime}\right) \mathbf{x}^{\prime}=\mathbf{u}$ and $\phi_{2}\left(\mathbf{x}^{\prime}, \mathbf{y}^{\prime}\right) \mathbf{y}^{\prime}=\mathbf{v}$. Note that

$$
0=\langle\mathbf{u}, \mathbf{v}\rangle=\left\langle\phi_{1}\left(\mathbf{x}^{\prime}, \mathbf{y}^{\prime}\right) \mathbf{x}^{\prime}, \phi_{2}\left(\mathbf{x}^{\prime}, \mathbf{y}^{\prime}\right) \mathbf{y}^{\prime}\right\rangle=\phi_{1}\left(\mathbf{x}^{\prime}, \mathbf{y}^{\prime}\right) \phi_{2}\left(\mathbf{x}^{\prime}, \mathbf{y}^{\prime}\right)\left\langle\mathbf{x}^{\prime}, \mathbf{y}^{\prime}\right\rangle,
$$


which implies $\left\langle\mathbf{x}^{\prime}, \mathbf{y}^{\prime}\right\rangle=0$ since $\phi_{1}\left(\mathbf{x}^{\prime}, \mathbf{y}^{\prime}\right) \neq 0$ and $\phi_{2}\left(\mathbf{x}^{\prime}, \mathbf{y}^{\prime}\right) \neq 0$. It follows that

$$
\phi_{1}\left(\mathbf{x}^{\prime}, \mathbf{y}^{\prime}\right)=\frac{1+\left\|\mathbf{y}^{\prime}\right\|^{2}}{1+\left\|\mathbf{x}^{\prime}\right\|^{2}\left\|\mathbf{y}^{\prime}\right\|^{2}} \quad \text { and } \quad \phi_{2}\left(\mathbf{x}^{\prime}, \mathbf{y}^{\prime}\right)=\frac{1-\left\|\mathbf{x}^{\prime}\right\|^{2}}{1+\left\|\mathbf{x}^{\prime}\right\|^{2}\left\|\mathbf{y}^{\prime}\right\|^{2}}
$$

Further, $\phi_{1}\left(\mathbf{x}^{\prime}, \mathbf{y}^{\prime}\right)\left\|\mathbf{x}^{\prime}\right\|=\|\mathbf{u}\|$ and $\phi_{2}\left(\mathbf{x}^{\prime}, \mathbf{y}^{\prime}\right)\left\|\mathbf{y}^{\prime}\right\|=\|\mathbf{v}\|$. If $\mathbf{v}=\mathbf{0}$, we have $\mathbf{y}^{\prime}=\mathbf{0}$ since $\phi_{2}\left(\mathbf{x}^{\prime}, \mathbf{y}^{\prime}\right) \neq$ 0 . This implies $\phi_{1}\left(\mathbf{x}^{\prime}, \mathbf{y}^{\prime}\right)=1$ and so $\mathbf{x}^{\prime}=\mathbf{u}$. In this case, $\left(\mathbf{x}^{\prime}, \mathbf{y}^{\prime}\right)=(\mathbf{u}, \mathbf{0})=\left(\lambda_{1} \mathbf{u}, \lambda_{2} \mathbf{v}\right)$. If $\mathbf{v} \neq \mathbf{0}$ and $\mathbf{u}=\mathbf{0}$, we have $\mathbf{x}^{\prime}=\mathbf{0}$. Hence $\phi_{2}\left(\mathbf{x}^{\prime}, \mathbf{y}^{\prime}\right)=1$ and so $\mathbf{y}^{\prime}=\mathbf{v}$. In this case, $\left(\mathbf{x}^{\prime}, \mathbf{y}^{\prime}\right)=(\mathbf{0}, \mathbf{v})=\left(\lambda_{1} \mathbf{u}, \lambda_{2} \mathbf{v}\right)$. Therefore, we may assume that $\mathbf{u} \neq \mathbf{0}$ and $\mathbf{v} \neq \mathbf{0}$. Set

$$
\mu_{1}=\frac{1}{\phi_{1}\left(\mathbf{x}^{\prime}, \mathbf{y}^{\prime}\right)} \quad \text { and } \quad \mu_{2}=\frac{1}{\phi_{2}\left(\mathbf{x}^{\prime}, \mathbf{y}^{\prime}\right)} .
$$

Then $\mu_{1}, \mu_{2}>0$ and $\mathbf{x}^{\prime}=\mu_{1} \mathbf{u}$ and $\mathbf{y}^{\prime}=\mu_{2} \mathbf{v}$. Direct computation shows that $1+\mu_{2}\|\mathbf{v}\|^{2}=$ $\frac{1}{\mu_{1}}$. Furthermore, $\phi_{1}\left(\mathbf{x}^{\prime}, \mathbf{y}^{\prime}\right) \mathbf{x}^{\prime}=\mathbf{u}, \mathbf{x}^{\prime}=\mu_{1} \mathbf{u}$, and $\mathbf{y}^{\prime}=\mu_{2} \mathbf{v}$ together imply $\phi_{1}\left(\mu_{1} \mathbf{u}, \mu_{2} \mathbf{v}\right) \mu_{1} \mathbf{u}=\mathbf{u}$. This in turn implies $\phi_{1}\left(\mu_{1} \mathbf{u}, \mu_{2} \mathbf{v}\right) \mu_{1}=1$ since $\mathbf{u} \neq \mathbf{0}$. Hence, $\frac{\mu_{1}\left(1+\mu_{2}^{2}\|\mathbf{v}\|^{2}\right)}{1+\mu_{1}^{2} \mu_{2}^{2}\|\mathbf{u}\|^{2}\|\mathbf{v}\|^{2}}=1$ and we obtain that $\|\mathbf{v}\|^{2} \mu_{2}^{2}+\left(1-\left(\|\mathbf{u}\|^{2}+\|\mathbf{v}\|^{2}\right)\right) \mu_{2}-1=0$. It follows that $\mu_{2}$ is a solution to the quadratic equation

$$
\|\mathbf{v}\|^{2} x^{2}+\left(1-\left(\|\mathbf{u}\|^{2}+\|\mathbf{v}\|^{2}\right)\right) x-1=0 .
$$

$$
\text { If } \mu_{2}=\frac{2}{1-\|\mathbf{u}\|^{2}-\|\mathbf{v}\|^{2}-\sqrt{\left(1-\left(\|\mathbf{u}\|^{2}+\|\mathbf{v}\|^{2}\right)\right)^{2}+4\|\mathbf{v}\|^{2}}} \text { were true, we would have }
$$

$$
\mu_{2}=-\frac{2}{-1+\|\mathbf{u}\|^{2}+\|\mathbf{v}\|^{2}+\sqrt{\left(1-\left(\|\mathbf{u}\|^{2}+\|\mathbf{v}\|^{2}\right)\right)^{2}+4\|\mathbf{v}\|^{2}}}<0
$$

which is a contradiction. Hence, it must be the case that

$$
\mu_{2}=\frac{2}{\left.\left.1-\|\mathbf{u}\|^{2}+\|\mathbf{v}\|^{2}+\sqrt{\left(1-\left(\|\mathbf{u}\|^{2}+\|\mathbf{v}\|^{2}\right.\right.}\right)\right)^{2}+4\|\mathbf{v}\|^{2}}=\lambda_{2}
$$

This in turn implies $\mu_{1}=\frac{1}{1+\mu_{2}\|\mathbf{v}\|^{2}}=\frac{1}{1+\lambda_{2}\|\mathbf{v}\|^{2}}=\lambda_{1}$. Hence, $\left(\mathbf{x}^{\prime}, \mathbf{y}^{\prime}\right)=\left(\lambda_{1} \mathbf{u}, \lambda_{2} \mathbf{v}\right)$. This proves uniqueness of the solution. Therefore, the Möbius gyrogroup satisfies (H2).

Now, let $P$ be a linear subspace of $\mathbb{R}^{n}$ and $P_{\mathbb{B}}=P \cap \mathbb{B}$ and $P_{\mathbb{B}}^{\perp}=P^{\perp} \cap \mathbb{B}$. The next three theorems are consequences of Theorems 5, 8, 10,13 obtained in Section 3.

Theorem 22. Let $P_{\mathbb{B}}$ be a subgyrogroup of $\left(\mathbb{B}, \oplus_{M}\right)$. For all $\mathbf{w} \in \mathbb{B}$ such that $\mathbf{w}=\mathbf{u}+\mathbf{v}$, with $\mathbf{u} \in P_{\mathbb{B}}$ and $\mathbf{v} \in P_{\mathbb{B}}^{\perp}$, the unique orthogonal decompositions of $\mathbf{w}$ according to $\oplus_{M}$ are given by

$$
\mathbf{w}=\lambda_{1}(\mathbf{u}, \mathbf{v}) \mathbf{u} \oplus_{M} \lambda_{2}(\mathbf{u}, \mathbf{v}) \mathbf{v} \text { and } \quad \mathbf{w}=\lambda_{3}(\mathbf{u}, \mathbf{v}) \mathbf{v} \oplus_{M} \lambda_{4}(\mathbf{u}, \mathbf{v}) \mathbf{u},
$$

where

$$
\begin{aligned}
& \lambda_{1}(\mathbf{u}, \mathbf{v})=\frac{2}{1+\|\mathbf{u}\|^{2}+\|\mathbf{v}\|^{2}+\sqrt{\left(1-\left(\|\mathbf{u}\|^{2}+\|\mathbf{v}\|^{2}\right)\right)^{2}+4\|\mathbf{v}\|^{2}}}, \\
& \lambda_{2}(\mathbf{u}, \mathbf{v})=\frac{2}{1-\|\mathbf{u}\|^{2}-\|\mathbf{v}\|^{2}+\sqrt{\left(1-\left(\|\mathbf{u}\|^{2}+\|\mathbf{v}\|^{2}\right)\right)^{2}+4\|\mathbf{v}\|^{2}}} \\
& \lambda_{3}(\mathbf{u}, \mathbf{v})=\frac{2}{1+\|\mathbf{u}\|^{2}+\|\mathbf{v}\|^{2}+\sqrt{\left(1-\left(\|\mathbf{u}\|^{2}+\|\mathbf{v}\|^{2}\right)\right)^{2}+4\|\mathbf{u}\|^{2}}}, \\
& \lambda_{4}(\mathbf{u}, \mathbf{v})=\frac{2}{1-\|\mathbf{u}\|^{2}-\|\mathbf{v}\|^{2}+\sqrt{\left(1-\left(\|\mathbf{u}\|^{2}+\|\mathbf{v}\|^{2}\right)\right)^{2}+4\|\mathbf{u}\|^{2}}}
\end{aligned}
$$


We remark that the special cases $\mathbf{u}=\mathbf{0}$ or $\mathbf{v}=\mathbf{0}$ are included in the general solution (63). As a consequence of Theorem 22, for each $\mathbf{w} \in \mathbb{B}$, if $\mathbf{w}=\mathbf{u}+\mathbf{v}$, with $\mathbf{u} \in P$ and $\mathbf{v} \in P^{\perp}$ is the unique decomposition of $\mathbf{w}$, according to the orthogonal direct sum decomposition $\mathbb{R}^{n}=P \oplus P^{\perp}$, then the orthogonal gyroprojections of the Möbius gyrogroup $\left(\mathbb{B}, \oplus_{M}\right)$ onto the subgyrogroups $P_{\mathbb{B}}$ and $P_{\mathbb{B}}^{\perp}$ are given by

$$
\begin{array}{ll}
\mathcal{P}_{M}^{\ell}: \mathbb{B}=P_{\mathbb{B}} \oplus_{M} P_{\mathbb{B}}^{\perp} \rightarrow P_{\mathbb{B}}, \quad \mathcal{P}_{M}^{\ell}(\mathbf{w})=\frac{2}{1+\|\mathbf{w}\|^{2}+\sqrt{\left(1-\|\mathbf{w}\|^{2}\right)^{2}+4\|\mathbf{v}\|^{2}}} \mathbf{u} \\
\mathcal{Q}_{M}^{r}: \mathbb{B}=P_{\mathbb{B}} \oplus_{M} P_{\mathbb{B}}^{\perp} \rightarrow P_{\mathbb{B}}^{\perp}, \quad \mathcal{Q}_{M}^{r}(\mathbf{w})=\frac{2}{1-\|\mathbf{w}\|^{2}+\sqrt{\left(1-\|\mathbf{w}\|^{2}\right)^{2}+4\|\mathbf{v}\|^{2}}} \mathbf{v} \\
\mathcal{Q}_{M}^{\ell}: \mathbb{B}=P_{\mathbb{B}}^{\perp} \oplus_{M} P_{\mathbb{B}} \rightarrow P_{\mathbb{B}}^{\perp}, \quad \mathcal{Q}_{M}^{\ell}(\mathbf{w})=\frac{2}{1+\|\mathbf{w}\|^{2}+\sqrt{\left(1-\|\mathbf{w}\|^{2}\right)^{2}+4\|\mathbf{u}\|^{2}}} \mathbf{v} \\
\mathcal{P}_{M}^{r}: \mathbb{B}=P_{\mathbb{B}}^{\perp} \oplus_{M} P_{\mathbb{B}} \rightarrow P_{\mathbb{B},}, \quad \mathcal{P}_{M}^{r}(\mathbf{w})=\frac{2}{1-\|\mathbf{w}\|^{2}+\sqrt{\left(1-\|\mathbf{w}\|^{2}\right)^{2}+4\|\mathbf{u}\|^{2}}} \mathbf{u} .
\end{array}
$$

Theorem 23. Let $P_{\mathbb{B}}$ be a subgyrogroup of $\left(\mathbb{B}, \oplus_{M}\right)$. Then the sets $\left\{b \oplus_{M} P_{\mathbb{B}}: b \in P_{\mathbb{B}}^{\perp}\right\}$ and $\left\{P_{\mathbb{B}} \oplus_{M} b: b \in P_{\mathbb{B}}^{\perp}\right\}$ are disjoint partitions of $\mathbb{B}$, that is,

$$
\mathbb{B}=\bigcup_{b \in P_{\mathbb{B}}^{\perp}}\left(b \oplus_{M} P_{\mathbb{B}}\right) \quad \text { and } \quad \mathbb{B}=\bigcup_{b \in P_{\mathbb{B}}^{\perp}}\left(P_{\mathbb{B}} \oplus_{M} b\right) .
$$

Moreover, $\left(\mathbb{B} / P_{\mathbb{B}}, \sim_{\ell}\right)=\left\{b \oplus_{M} P_{\mathbb{B}}: b \in P_{\mathbb{B}}^{\perp}\right\}$ and $\left(\mathbb{B} / P_{\mathbb{B}}, \sim_{r}\right)=\left\{P_{\mathbb{B}} \oplus_{M} b: b \in P_{\mathbb{B}}^{\perp}\right\}$.

Theorem 24. Let $P_{\mathbb{B}}$ be a subgyrogroup of $\left(\mathbb{B}, \oplus_{M}\right)$. Then the following are gyrogroup isomorphisms:

$$
\left(\mathbb{B} / P_{\mathbb{B}}, \sim_{\ell}\right) \cong\left(P_{\mathbb{B}}^{\perp}, \oplus_{M}\right) \cong\left(\mathbb{B} / P_{\mathbb{B}}, \sim_{r}\right) .
$$

For each positive number $t$, recall that the $t$-Möbius addition is defined by

$$
\mathbf{u} \oplus_{M_{t}} \mathbf{v}=\frac{\left(1+\frac{2}{t^{2}}\langle\mathbf{u}, \mathbf{v}\rangle+\frac{1}{t^{2}}\|\mathbf{v}\|^{2}\right) \mathbf{u}+\left(1-\frac{1}{t^{2}}\|\mathbf{u}\|^{2}\right) \mathbf{v}}{1+\frac{2}{t^{2}}\langle\mathbf{u}, \mathbf{v}\rangle+\frac{1}{t^{4}}\|\mathbf{u}\|^{2}\|\mathbf{v}\|^{2}}
$$

for all $\mathbf{u}, \mathbf{v} \in \mathbb{B}_{t}$. Let $P$ be a subspace of $\mathbb{R}^{n}$. For each $\mathbf{w} \in \mathbb{B}_{t}$, if $\mathbf{w}=\mathbf{u}+\mathbf{v}$ is the unique expression of $\mathbf{w}$, according to the orthogonal direct sum decomposition $\mathbb{R}^{n}=P \oplus P^{\perp}$, with $\mathbf{u} \in P$ and $\mathbf{v} \in P^{\perp}$, then the orthogonal gyroprojections of the $t$-Möbius gyrogroup (after rescaling $u \mapsto \frac{u}{t}, v \mapsto \frac{v}{t}, w \mapsto \frac{w}{t}$ ) are given by

$$
\begin{aligned}
& \mathcal{P}_{M_{t}}^{\ell}(\mathbf{w})=\frac{2}{1+\frac{\|\mathbf{w}\|^{2}}{t^{2}}+\sqrt{\left(1-\frac{\|\mathbf{w}\|^{2}}{t^{2}}\right)^{2}+4 \frac{\|\mathbf{v}\|^{2}}{t^{2}}}} \mathbf{u}, \\
& \mathcal{P}_{M_{t}}^{r}(\mathbf{w})=\frac{2}{1-\frac{\|\mathbf{w}\|^{2}}{t^{2}}+\sqrt{\left(1-\frac{\|\mathbf{w}\|^{2}}{t^{2}}\right)^{2}+4 \frac{\|\mathbf{u}\|^{2}}{t^{2}}}} \mathbf{u}, \\
& \mathcal{Q}_{M_{t}}^{\ell}(\mathbf{w})=\frac{2}{1+\frac{\|\mathbf{w}\|^{2}}{t^{2}}+\sqrt{\left(1-\frac{\|\mathbf{w}\|^{2}}{t^{2}}\right)^{2}+4 \frac{\|\mathbf{u}\|^{2}}{t^{2}}}} \mathbf{v} \\
& \mathcal{Q}_{M_{t}}^{r}(\mathbf{w})=\frac{2}{1-\frac{\|\mathbf{w}\|^{2}}{t^{2}}+\sqrt{\left(1-\frac{\|\mathbf{w}\|^{2}}{t^{2}}\right)^{2}+4 \frac{\|\mathbf{v}\|^{2}}{t^{2}}}} \mathbf{v}
\end{aligned}
$$


When $t \rightarrow+\infty, \oplus_{M_{t}}$ reduces to ordinary vector addition of $\mathbb{R}^{n}, \mathcal{P}_{M_{t}}^{\ell}$ and $\mathcal{P}_{M_{t}}^{r}$ reduce to the ordinary projection $\mathcal{P}(\mathbf{u}+\mathbf{v})=\mathbf{u}$, and $\mathcal{Q}_{M_{t}}^{\ell}$ and $\mathcal{Q}_{M_{t}}^{r}$ reduce to the ordinary projection $\mathcal{Q}(\mathbf{u}+\mathbf{v})=\mathbf{v}$. Further, the equivalence relation (29) reflects the Euclidean left coset relation:

$$
\mathbf{u} \sim_{\ell} \mathbf{v} \quad \text { if and only if } \quad-\mathbf{v}+\mathbf{u} \in P
$$

and the equivalence relation (31) reflects the Euclidean right coset relation:

$$
\mathbf{u} \sim_{r} \mathbf{v} \quad \text { if and only if } \quad \mathbf{u}-\mathbf{v} \in P .
$$

Now, we analyze the fiber bundles and the sections of the Möbius gyrogroup arisen from the two orthogonal decompositions $\mathbb{B}=P_{\mathbb{B}} \oplus_{M} P_{\mathbb{B}}^{\perp}$ and $\mathbb{B}=P_{\mathbb{B}}^{\perp} \oplus_{M} P_{\mathbb{B}}$. First we give the relation between these two decompositions according to Theorem 6.

Theorem 25. Let $\mathbf{u}, \mathbf{v} \in\left(\mathbb{B}, \oplus_{M}\right)$ such that $\langle\mathbf{u}, \mathbf{v}\rangle=0$. Then

$$
\mathbf{u} \oplus_{M} \mathbf{v}=\mu_{1}(\mathbf{u}, \mathbf{v}) \mathbf{v} \oplus_{M} \mu_{2}(\mathbf{u}, \mathbf{v}) \mathbf{u},
$$

where

$$
\begin{aligned}
& \mu_{1}(\mathbf{u}, \mathbf{v})=\frac{2\left(1-\|\mathbf{u}\|^{2}\right)}{\left(1+\|\mathbf{u}\|^{2}\right)\left(1+\|\mathbf{v}\|^{2}\right)+\sqrt{(1-\|\mathbf{u}\|)^{2}(1-\|\mathbf{v}\|)^{2}+4\left(1+\|\mathbf{v}\|^{2}\right)\|u\|^{2}}} \\
& \mu_{2}(\mathbf{u}, \mathbf{v})=\frac{2\left(1+\|\mathbf{v}\|^{2}\right)}{\left(1-\|\mathbf{u}\|^{2}\right)\left(1-\|\mathbf{v}\|^{2}\right)+\sqrt{(1-\|\mathbf{u}\|)^{2}(1-\|\mathbf{v}\|)^{2}+4\left(1+\|\mathbf{v}\|^{2}\right)\|u\|^{2}}} .
\end{aligned}
$$

Proof. By (27), (56), (49), and straightforward computations we have:

$$
\begin{aligned}
\mu_{1}(\mathbf{u}, \mathbf{v}) & =\lambda_{3}\left(\phi_{1}(\mathbf{u}, \mathbf{v}) \mathbf{u}, \phi_{2}(\mathbf{u}, \mathbf{v}) \mathbf{v}\right) \phi_{2}(\mathbf{u}, \mathbf{v}) \\
& =\lambda_{3}\left(\frac{1+\|\mathbf{v}\|^{2}}{1+\|\mathbf{u}\|^{2}\|\mathbf{v}\|^{2}} \mathbf{u}, \frac{1-\|\mathbf{u}\|^{2}}{1+\|\mathbf{u}\|^{2}\|\mathbf{v}\|^{2}} \mathbf{v}\right) \frac{1-\|\mathbf{u}\|^{2}}{1+\|\mathbf{u}\|^{2}\|\mathbf{v}\|^{2}} \\
& =\frac{2\left(1-\|\mathbf{u}\|^{2}\right)}{\left(1+\|\mathbf{u}\|^{2}\right)\left(1+\|\mathbf{v}\|^{2}\right)+\sqrt{(1-\|\mathbf{u}\|)^{2}(1-\|\mathbf{v}\|)^{2}+4\left(1+\|\mathbf{v}\|^{2}\right)\|\mathbf{u}\|^{2}}} \\
\mu_{2}(\mathbf{u}, \mathbf{v}) & =\lambda_{4}\left(\phi_{1}(\mathbf{u}, \mathbf{v}) \mathbf{u}, \phi_{2}(\mathbf{u}, \mathbf{v}) \mathbf{v}\right) \phi_{1}(\mathbf{u}, \mathbf{v}) \\
& =\lambda_{4}\left(\frac{1+\|\mathbf{v}\|^{2}}{1+\|\mathbf{u}\|^{2}\|\mathbf{v}\|^{2}} \mathbf{u}, \frac{1-\|\mathbf{u}\|^{2}}{1+\|\mathbf{u}\|^{2}\|\mathbf{v}\|^{2}} \mathbf{v}\right) \frac{1+\|\mathbf{v}\|^{2}}{1+\|\mathbf{u}\|^{2}\|\mathbf{v}\|^{2}} \\
& =\frac{2\left(1+\|\mathbf{v}\|^{2}\right)}{\left(1-\|\mathbf{u}\|^{2}\right)\left(1-\|\mathbf{v}\|^{2}\right)+\sqrt{(1-\|\mathbf{u}\|)^{2}(1-\|\mathbf{v}\|)^{2}+4\left(1+\|\mathbf{v}\|^{2}\right)\|\mathbf{u}\|^{2}}}
\end{aligned}
$$

Now, we analyze the properties of $\mu_{1}(\mathbf{u}, \mathbf{v})\|\mathbf{v}\|$ and $\mu_{2}(\mathbf{v}, \mathbf{u})\|\mathbf{v}\|$. For any $\mathbf{u} \in P_{\mathbb{B}} \backslash\{\mathbf{0}\}$ we have that $\mu_{1}(\mathbf{u}, \mathbf{v})\|\mathbf{v}\|$ is a strictly increasing function such that $\mu_{1}(\mathbf{u}, \mathbf{0})\|\mathbf{0}\|=0$ and $\lim _{\|\mathbf{v}\| \rightarrow 1} \mu_{1}(\mathbf{u}, \mathbf{v})\|\mathbf{v}\|=$ $\frac{1-\|\mathbf{u}\|^{2}}{1+\sqrt{2}\|\mathbf{u}\|+\|\mathbf{u}\|^{2}}$. Therefore, for any $\mathbf{u} \in P_{\mathbb{B}} \backslash\{\mathbf{0}\}$ it follows that $\mu_{1}(\mathbf{u}, \mathbf{v})\|\mathbf{v}\| \in\left[0, \frac{1-\|\mathbf{u}\|^{2}}{1+\sqrt{2}\|\mathbf{u}\|+\|\mathbf{u}\|^{2}}[\subsetneq[0,1[\right.$ for all $\mathbf{v} \in P_{\mathbb{B}}^{\perp}$ and, consequently, $\left\{\mu_{1}(\mathbf{u}, \mathbf{v}) \mathbf{v}: \mathbf{v} \in P_{\mathbb{B}}^{\perp}\right\} \subsetneq P_{\mathbb{B}}^{\perp}$. Then for any $\mathbf{u} \in P_{\mathbb{B}} \backslash\{\mathbf{0}\}, \tau_{\mathbf{u}}^{(2)}$ is a local section for the fiber bundles $\pi_{1}$ and $\pi_{3}$ defined in (38). In the case when $\mathbf{u}=\mathbf{0}$ the section $\tau_{\mathbf{0}}^{(2)}$ is a global section as seen in (41).

Concerning $\mu_{2}(\mathbf{v}, \mathbf{u})\|\mathbf{v}\|$ it turns out that for each $\mathbf{u} \in P_{\mathbb{B}}$, the function $\mu_{2}(\mathbf{v}, \mathbf{u})\|\mathbf{v}\|$ is strictly increasing such that $\mu_{2}(\mathbf{0}, \mathbf{u})\|\mathbf{0}\|=0$ and $\lim _{\|\mathbf{v}\| \rightarrow 1} \mu_{2}(\mathbf{v}, \mathbf{u})\|\mathbf{v}\|=1$. Therefore, we conclude that $\left\{\mu_{2}(\mathbf{v}, \mathbf{u}) \mathbf{v}: \mathbf{v} \in P_{\mathbb{B}}^{\perp}\right\}=P_{\mathbb{B}}^{\perp}$ and, consequently, for any $\mathbf{u} \in P_{\mathbb{B}}$ the section $\tau_{\mathbf{u}}^{(4)}$ is a global section for the fiber bundles $\pi_{2}$ and $\pi_{4}$ defined in (38). 
In Figure 4 we show the plots of $\mu_{1}(\mathbf{u}, \mathbf{v})\|\mathbf{v}\|$ and $\mu_{2}(\mathbf{v}, \mathbf{u})\|\mathbf{v}\|$, with $\|\mathbf{u}\|,\|\mathbf{v}\|<1$, for the case of the Möbius gyrogroup.

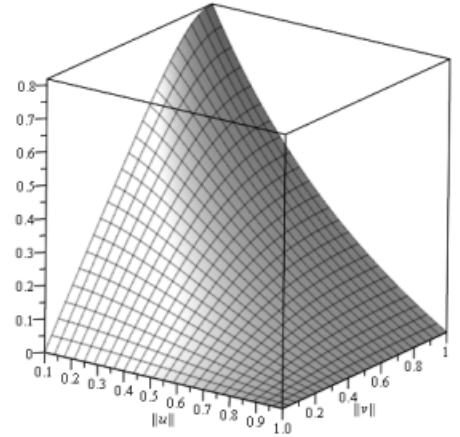

(a)

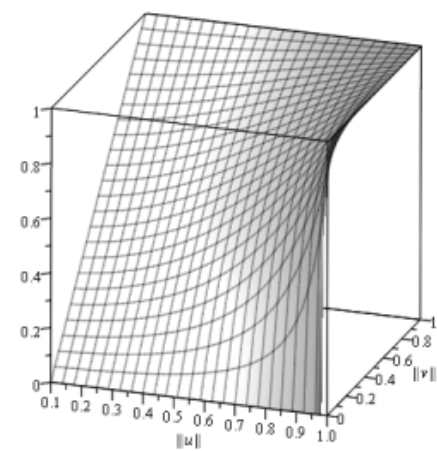

(b)

Figure 4. (a) Plot of $\mu_{1}(\mathbf{u}, \mathbf{v})\|\mathbf{v}\|$ with $\|\mathbf{u}\|,\|\mathbf{v}\|<1$; (b) plot of $\mu_{2}(\mathbf{v}, \mathbf{u})\|\mathbf{v}\|$ with $\|\mathbf{u}\|,\|\mathbf{v}\|<1$, for the Möbius gyrogroup.

Theorem 21 about the duality relations between left and right cosets obtained from the orthogonal factorization of $\mathbb{B}$ by $P_{\mathbb{B}}$ and by $P_{\mathbb{B}}^{\perp}$, holds also in the case of the Möbius gyrogroup. Analogously to the Einstein case we restrict now to the 3-dimensional space and we show in Figures 5 and 6 the left and right cosets obtained from the orthogonal decompositions of the gyrogroup $\left(\mathbb{B}^{3}, \oplus_{M}\right)$ by the subgyrogroups $L_{e_{3}}^{\mathbb{B}^{3}}$ and $D_{e_{3}}^{\mathbb{B}^{3}}$.

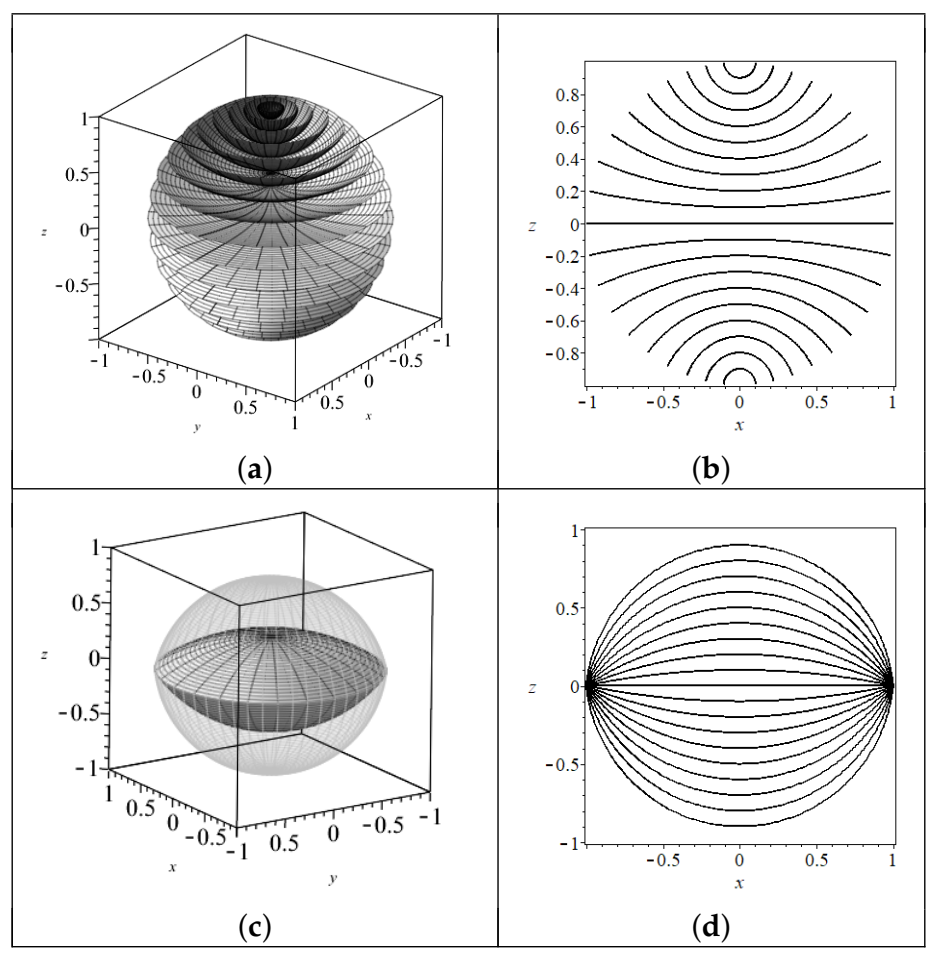

Figure 5. (a) Left cosets $\left(\mathbb{B}^{3} / D_{e_{3}}^{\mathbb{B}^{3}}, \sim_{\ell}\right)=\left\{\mathbf{u} \oplus_{M} D_{\mathfrak{e}_{3}}^{\mathbb{B}^{3}}, \mathbf{u} \in L_{\mathfrak{e}_{3}}^{\mathbb{B}^{3}}\right\} ;(\mathbf{b})$ gyrolines in the Möbius gyrovector plane (projection in the $x z$-plane of left cosets); (c) right cosets $\left(\mathbb{B}^{3} / D_{e_{3}}^{\mathbb{B}_{3}^{3}}, \sim_{r}\right)=\left\{D_{e_{3}}^{\mathbb{B}^{3}} \oplus_{M} \mathbf{u}, \mathbf{u} \in L_{e_{3}}^{\mathbb{B}^{3}}\right\}$; (d) cogyrolines in the Möbius gyrovector plane (projection in the $x z$-plane of right cosets). 


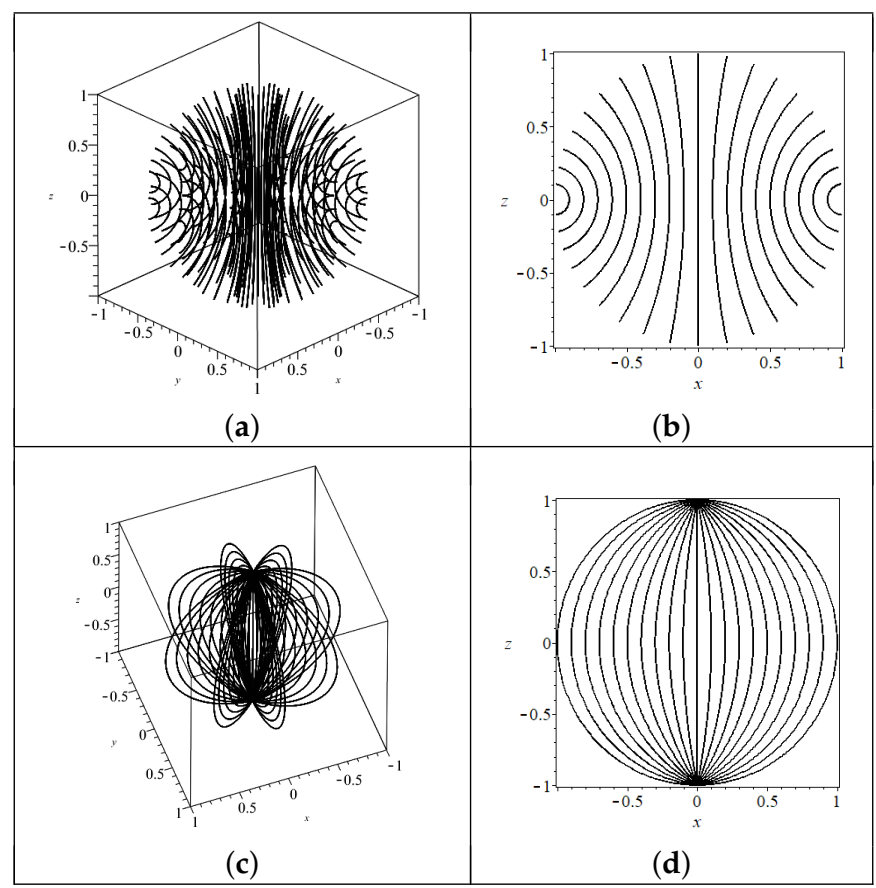

Figure 6. (a) Left cosets $\left(\mathbb{B}^{3} / L_{\mathbb{E}_{3}}^{\mathbb{B}^{3}}, \sim_{\ell}\right)=\left\{\mathbf{v} \oplus_{M} L_{e_{3}}^{\mathbb{B}^{3}}, \mathbf{v} \in D_{\mathfrak{e}_{3}}^{\mathbb{B}^{3}}\right\} ;$ (b) gyrolines in the Möbius gyrovector plane (projection in the $x z$-plane of left cosets); (c) right cosets $\left(\mathbb{B}^{3} / L_{e_{3}}^{\mathbb{B}^{3}}, \sim_{r}\right)=\left\{L_{e_{3}}^{\mathbb{B}^{3}} \oplus_{M} \mathbf{v}, \mathbf{v} \in D_{e_{3}}^{\mathbb{B}^{3}}\right\}$; (d) cogyrolines in the Möbius gyrovector plane (projection in the $x z$-plane of right cosets).

\subsection{Proper Velocity Gyrogroup}

The (Euclidean) Proper Velocity (PV) gyrogroup [3] consists of the $n$-dimensional Euclidean space $\mathbb{R}^{n}$ endowed with PV addition $\oplus u$ defined by

$$
\mathbf{u} \oplus U \mathbf{v}=\left(\frac{\beta_{\mathbf{u}}}{1+\beta_{\mathbf{u}}}\langle\mathbf{u}, \mathbf{v}\rangle+\frac{1}{\beta_{\mathbf{v}}}\right) \mathbf{u}+\mathbf{v}
$$

for all $\mathbf{u}, \mathbf{v} \in \mathbb{R}^{n}$, where $\beta_{\mathbf{u}}$ is the beta factor given by $\beta_{\mathbf{u}}=\frac{1}{\sqrt{1+\|\mathbf{u}\|^{2}}}$ for all $\mathbf{u} \in \mathbb{R}^{n}$. In view of (70), we define

$$
\phi_{1}(\mathbf{u}, \mathbf{v})=\frac{\beta_{\mathbf{u}}}{1+\beta_{\mathbf{u}}}\langle\mathbf{u}, \mathbf{v}\rangle+\frac{1}{\beta_{\mathbf{v}}} \quad \text { and } \quad \phi_{2}(\mathbf{u}, \mathbf{v})=1
$$

for all $\mathbf{u}, \mathbf{v} \in \mathbb{R}^{n}$. The gyrogroup identity of $\left(\mathbb{R}^{n}, \oplus_{U}\right)$ is the zero vector $\mathbf{0}$. Further, $\ominus_{U} \mathbf{u}=-\mathbf{u}$ for all $\mathbf{u} \in \mathbb{R}^{n}$. Let $\mathbf{u}, \mathbf{v} \in \mathbb{R}^{n}$. The PV addition satisfies the beta identity given by

$$
\frac{1}{\beta_{\mathbf{u} \oplus u \mathbf{v}}}=\frac{1}{\beta_{\mathbf{u}} \beta_{\mathbf{v}}}+\langle\mathbf{u}, \mathbf{v}\rangle=\frac{1+\beta_{\mathbf{u}} \beta_{\mathbf{v}}\langle\mathbf{u}, \mathbf{v}\rangle}{\beta_{\mathbf{u}} \beta_{\mathbf{v}}}
$$

Hence, $1+\beta_{\mathbf{u}} \beta_{\mathbf{v}}\langle\mathbf{u}, \mathbf{v}\rangle>0$ since $\frac{\beta_{\mathbf{u}} \beta_{\mathbf{v}}}{\beta_{\mathbf{u} \oplus_{U} \mathbf{v}}}>0$. This implies

$$
\phi_{1}(\mathbf{u}, \mathbf{v})=\frac{\beta_{\mathbf{u}}+\left(1+\beta_{\mathbf{u}} \beta_{\mathbf{v}}\langle\mathbf{u}, \mathbf{v}\rangle\right)}{\left(1+\beta_{\mathbf{u}}\right) \beta_{\mathbf{v}}}>0 .
$$


It is clear that the PV gyrogroup satisfies (H1). Suppose that $\mathbf{u}, \mathbf{v} \in \mathbb{R}^{n}$ and that $\langle\mathbf{u}, \mathbf{v}\rangle=0$. It is easy to see that the unique solution of the system of equations

$$
\left\{\begin{array}{l}
\phi_{1}\left(\lambda_{1} \mathbf{u}, \lambda_{2} \mathbf{v}\right) \lambda_{1} \mathbf{u}=\mathbf{u} \\
\phi_{2}\left(\lambda_{1} \mathbf{u}, \lambda_{2} \mathbf{v}\right) \lambda_{2} \mathbf{v}=\mathbf{v}
\end{array}\right.
$$

is given by $\lambda_{1}=\beta_{\mathbf{v}}$ and $\lambda_{2}=1$. Thus, the PV gyrogroup satisfies (H2) and we obtain the next three theorems immediately as consequences of Theorems 5, 8, 10, 13 in Section 3.

Theorem 26. Let $P$ be a subgyrogroup of $\left(\mathbb{R}^{n}, \oplus u\right)$. For all $\mathbf{w} \in \mathbb{R}^{n}$ such that $\mathbf{w}=\mathbf{u}+\mathbf{v}$, with $\mathbf{u} \in P$ and $\mathbf{v} \in P^{\perp}$, the unique orthogonal decompositions of $\mathbf{w}$ according to $\oplus_{U}$ are given by

$$
\mathbf{w}=\lambda_{1}(\mathbf{u}, \mathbf{v}) \mathbf{u} \oplus_{U} \lambda_{2}(\mathbf{u}, \mathbf{v}) \mathbf{v} \text { and } \mathbf{w}=\lambda_{3}(\mathbf{u}, \mathbf{v}) \mathbf{v} \oplus_{U} \lambda_{4}(\mathbf{u}, \mathbf{v}) \mathbf{u}
$$

where

$$
\begin{aligned}
& \lambda_{1}(\mathbf{u}, \mathbf{v})=\beta_{\mathbf{v}} \text { and } \lambda_{2}(\mathbf{u}, \mathbf{v})=1 \\
& \lambda_{3}(\mathbf{u}, \mathbf{v})=\lambda_{1}(\mathbf{v}, \mathbf{u})=\beta_{\mathbf{u}} \text { and } \lambda_{4}(\mathbf{u}, \mathbf{v})=1 .
\end{aligned}
$$

For each $\mathbf{w} \in \mathbb{R}^{n}$, if $\mathbf{w}=\mathbf{u}+\mathbf{v}$ is the unique expression of $\mathbf{w}$, according to the orthogonal direct sum decomposition $\mathbb{R}^{n}=P \oplus P^{\perp}$, then the orthogonal gyroprojections of the PV gyrogroup $\left(\mathbb{R}^{n}, \oplus U\right)$ onto the subgyrogroups $P$ and $P^{\perp}$ are given by

$$
\begin{array}{ll}
\mathcal{P}_{U}^{\ell}: \mathbb{R}^{n}=P \oplus_{U} P^{\perp} \rightarrow P, & \mathcal{P}_{U}^{\ell}(\mathbf{w})=\beta_{\mathbf{v}} \mathbf{u}, \\
\mathcal{Q}_{U}^{r}: \mathbb{R}^{n}=P \oplus_{U} P^{\perp} \rightarrow P^{\perp}, & \mathcal{Q}_{U}^{r}(\mathbf{w})=\mathbf{v}, \\
\mathcal{Q}_{U}^{\ell}: \mathbb{R}^{n}=P^{\perp} \oplus_{U} P \rightarrow P^{\perp}, & \mathcal{Q}_{U}^{\ell}(\mathbf{w})=\beta_{\mathbf{u}} \mathbf{v}, \\
\mathcal{P}_{U}^{r}: \mathbb{R}^{n}=P^{\perp} \oplus_{U} P \rightarrow P, & \mathcal{P}_{U}^{r}(\mathbf{w})=\mathbf{u} .
\end{array}
$$

Theorem 27. Let $P$ be a linear subspace of $\mathbb{R}^{n}$. Then the sets $\left\{b \oplus_{U} P: b \in P^{\perp}\right\}$ and $\left\{P \oplus_{U} b: b \in P^{\perp}\right\}$ are disjoint partitions of $\mathbb{R}^{n}$, that is,

$$
\mathbb{R}^{n}=\bigcup_{b \in P^{\perp}}\left(b \oplus_{U} P\right) \quad \text { and } \quad \mathbb{R}^{n}=\bigcup_{b \in P^{\perp}}\left(P \oplus_{U} b\right)
$$

Moreover, $\left(\mathbb{R}^{n} / P, \sim_{\ell}\right)=\left\{b \oplus_{U} P: b \in P^{\perp}\right\}$ and $\left(\mathbb{R}^{n} / P, \sim_{r}\right)=\left\{P \oplus_{U} b: b \in P^{\perp}\right\}$.

Theorem 28. Let $P$ be a linear subspace of $\mathbb{R}^{n}$. Then the following are gyrogroup isomorphisms:

$$
\left(\mathbb{R}^{n} / P, \sim_{\ell}\right) \cong\left(P^{\perp}, \oplus U\right) \cong\left(\mathbb{R}^{n} / P, \sim_{r}\right) .
$$

Recall that $t$-PV addition is defined by

$$
\mathbf{u} \oplus U_{t} \mathbf{v}=\left(\frac{\beta_{\mathbf{u}}}{1+\beta_{\mathbf{u}}} \frac{\langle\mathbf{u}, \mathbf{v}\rangle}{t^{2}}+\frac{1}{\beta_{\mathbf{v}}}\right) \mathbf{u}+\mathbf{v}
$$

for all $\mathbf{u}, \mathbf{v}, \in \mathbb{R}^{n}$, where $\beta_{\mathbf{u}}$ is redefined by $\beta_{\mathbf{u}}=\frac{1}{\sqrt{1+\frac{\|\mathbf{u}\|^{2}}{t^{2}}}}$. When $t$ is arbitrarily large (that is, $t \rightarrow+\infty), \oplus u_{t}$ reduces to ordinary vector addition of $\mathbb{R}^{n}$ and $\beta_{\mathbf{w}} \rightarrow 1$. Therefore, the orthogonal gyroprojections of the $t$-PV gyrogroup reduce to ordinary projection of $\mathbb{R}^{n}$ :

$$
P(\mathbf{u}+\mathbf{v})=\mathbf{u} \quad \text { and } \quad Q(\mathbf{u}+\mathbf{v})=\mathbf{v}
$$


for all $\mathbf{u} \in P, \mathbf{v} \in P^{\perp}$.

Now, we analyze the fiber bundles and sections of the PV gyrogroup arisen from the two orthogonal decompositions $\mathbb{R}^{n}=P \oplus_{U} P^{\perp}$ and $\mathbb{R}^{n}=P^{\perp} \oplus_{U} P$. First we give the relation between these two decompositions according to Theorem 6.

Theorem 29. Let $\mathbf{u}, \mathbf{v} \in\left(\mathbb{R}^{n}, \oplus_{U}\right)$ such that $\langle\mathbf{u}, \mathbf{v}\rangle=0$. Then

$$
\mathbf{u} \oplus_{U} \mathbf{v}=\mu_{1}(\mathbf{u}, \mathbf{v}) \mathbf{v} \oplus_{U} \mu_{2}(\mathbf{u}, \mathbf{v}) \mathbf{u},
$$

where

$$
\mu_{1}(\mathbf{u}, \mathbf{v})=\beta_{\frac{1}{\beta_{\mathbf{v}}}} \mathbf{u} \text { and } \mu_{2}(\mathbf{u}, \mathbf{v})=\frac{1}{\beta_{\mathbf{v}}} .
$$

Proof. By (27), (71), (73), we have

$$
\begin{aligned}
& \mu_{1}(\mathbf{u}, \mathbf{v})=\lambda_{3}\left(\phi_{1}(\mathbf{u}, \mathbf{v}) \mathbf{u}, \phi_{2}(\mathbf{u}, \mathbf{v}) \mathbf{v}\right) \phi_{2}(\mathbf{u}, \mathbf{v})=\lambda_{3}\left(\frac{1}{\beta_{\mathbf{v}}} \mathbf{u}, \mathbf{v}\right)=\beta_{\frac{1}{\beta_{\mathbf{v}}} \mathbf{u}^{\prime}} \\
& \mu_{2}(\mathbf{u}, \mathbf{v})=\lambda_{4}\left(\phi_{1}(\mathbf{u}, \mathbf{v}) \mathbf{u}, \phi_{2}(\mathbf{u}, \mathbf{v}) \mathbf{v}\right) \phi_{1}(\mathbf{u}, \mathbf{v})=\frac{1}{\beta_{\mathbf{v}}}
\end{aligned}
$$

In Figure 7 we show the plots of $\mu_{1}(\mathbf{u}, \mathbf{v})\|\mathbf{v}\|$ and $\mu_{2}(\mathbf{v}, \mathbf{u})\|\mathbf{v}\|$, with $\|\mathbf{u}\|,\|\mathbf{v}\|<1$, for the PV gyrogroup.

Since for any $\mathbf{u} \in P \backslash\{\mathbf{0}\}, \mu_{1}(\mathbf{u}, \mathbf{v})\|\mathbf{v}\|=\frac{\|\mathbf{v}\|}{\sqrt{1+\left(1+\|\mathbf{v}\|^{2}\right)\|\mathbf{u}\|^{2}}}$ is a strictly increasing function such that $\mu_{1}(\mathbf{u}, 0)\|\mathbf{0}\|=0$ and $\lim _{\|\mathbf{v}\| \rightarrow+\infty} \mu_{1}(\mathbf{u}, \mathbf{v})\|\mathbf{v}\|=\frac{1}{\|u\|}$, we conclude that $\mu_{1}(\mathbf{u}, \mathbf{v})\|\mathbf{v}\| \in\left[0, \frac{1}{\|\mathbf{u}\|}[\subsetneq\right.$ $\left[0,+\infty\left[\right.\right.$, for all $\mathbf{v} \in P^{\perp}$. Therefore, for any $\mathbf{u} \in P \backslash\{\mathbf{0}\}$, we have that $\left\{\mu_{1}(\mathbf{u}, \mathbf{v}) \mathbf{v}: \mathbf{v} \in P^{\perp}\right\} \subsetneq P^{\perp}$ and, consequently, $\tau_{\mathbf{u}}^{(2)}$ is a local section for the fiber bundles $\pi_{1}$ and $\pi_{3}$ defined in (38). In the case when $\mathbf{u}=\mathbf{0}$ the section $\tau_{\mathbf{0}}^{(2)}$ is a global section as seen in (41).

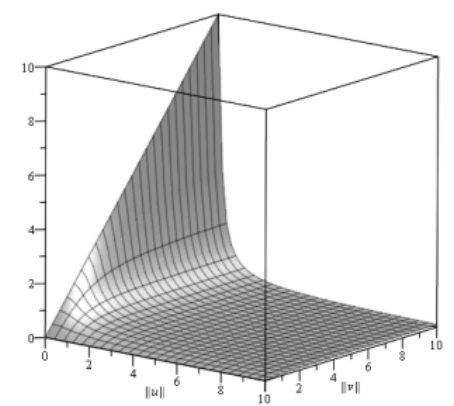

(a)

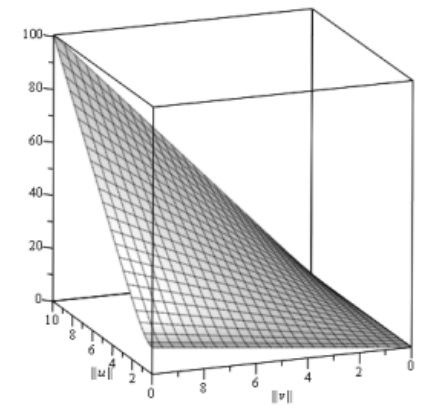

(b)

Figure 7. (a) Plot of $\mu_{1}(\mathbf{u}, \mathbf{v})\|\mathbf{v}\|$ with $\|u\|,\|v\| \in[0,10]$; (b) Plot of $\mu_{2}(\mathbf{v}, \mathbf{u})\|\mathbf{v}\|$ with $\|u\|,\|v\| \in[0,10]$, for the Proper Velocity (PV) gyrogroup.

Concerning the section $\tau_{\mathbf{u}}^{(4)}$, since for each $\mathbf{u} \in P$ we have that $\mu_{2}(\mathbf{v}, \mathbf{u})\|\mathbf{v}\|=\sqrt{1+\|\mathbf{v}\|}\left\|^{2}\right\| \mathbf{v} \|$ is a strictly increasing function in the variable $\|\mathbf{v}\|$ such that $\mu_{2}(\mathbf{0}, \mathbf{u})\|\mathbf{0}\|=0$ and $\lim _{\|\mathbf{v}\| \rightarrow+\infty} \mu_{2}(\mathbf{v}, \mathbf{u})\|\mathbf{v}\|=$ $+\infty$, we conclude that $\left\{\mu_{2}(\mathbf{v}, \mathbf{u}) \mathbf{v}: \mathbf{v} \in P^{\perp}\right\}=P^{\perp}$. Hence, for any $\mathbf{u} \in P$, it follows that $\tau_{\mathbf{u}}^{(4)}$ is a global section for the fiber bundles $\pi_{2}$ and $\pi_{4}$ defined in (38).

We can finally conclude that the same duality relations as in Theorem 21 happens for the case of the PV gyrogroup.

Theorem 30. Let $P$ be a subgyrogroup of $\left(\mathbb{R}^{n}, \oplus_{U}\right)$. The following duality relations hold:

1. The cosets of $\left(\mathbb{R}^{n} / P^{\perp}, \sim_{r}\right)$ are global sections for the quotient spaces $\left(\mathbb{R}^{n} / P, \sim_{\ell}\right)$ and $\left(\mathbb{R}^{n} / P, \sim_{r}\right)$, and vice versa. 
2. The cosets of $\left(\mathbb{R}^{n} / P^{\perp}, \sim_{\ell}\right)$ are global sections for the quotient space $\left(\mathbb{R}^{n} / P, \sim_{r}\right)$, and vice versa.

3. The cosets of $\left(\mathbb{R}^{n} / P^{\perp}, \sim_{\ell}\right)$ are local sections for the quotient space $\left(\mathbb{R}^{n} / P, \sim_{\ell}\right)$ except the identity coset $\mathbf{0} \oplus u P^{\perp}=P^{\perp}$ that is a global section, and vice versa.

To visualize the left and right cosets we restrict now to the 3-dimensional space $\mathbb{R}^{3}$ and we show in Figures 8 and 9 the cosets obtained from the orthogonal decompositions of the gyrogroup $\left(\mathbb{R}^{3}, \oplus_{V}\right)$ by the subgyrogroups $L_{e_{3}}$ and $D_{e_{3}}$.

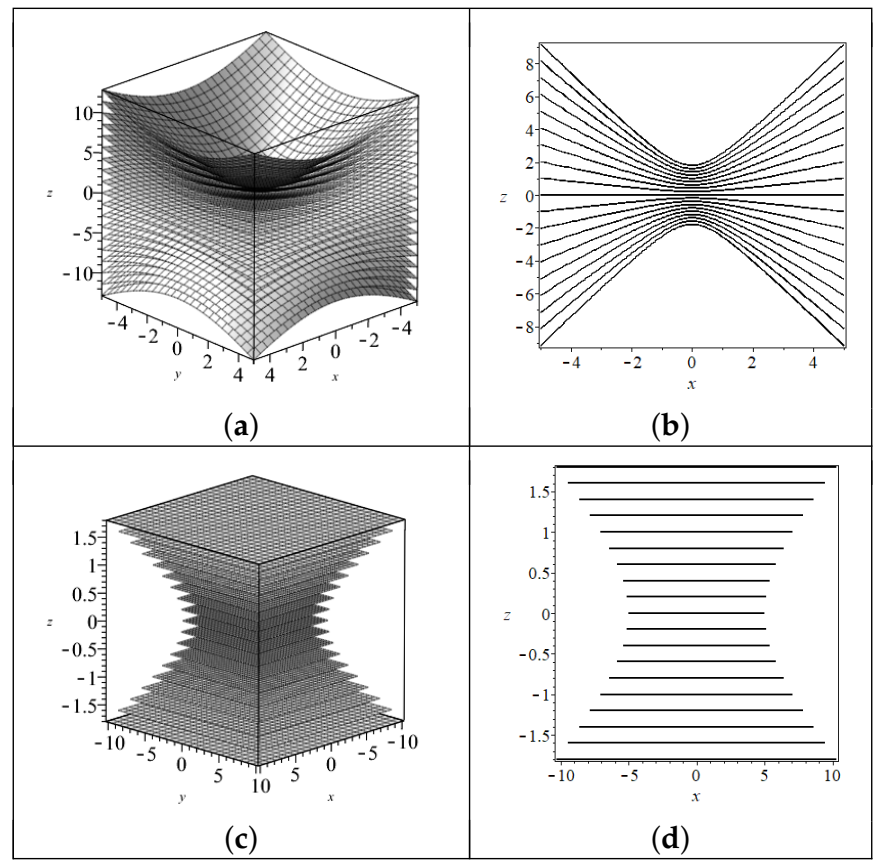

Figure 8. (a) Left cosets $\left(\mathbb{R}^{3} / D_{e_{3}}, \sim_{\ell}\right)=\left\{\mathbf{u} \oplus_{U} D_{e_{3}}, \mathbf{u} \in L_{e_{3}}\right\}$; (b) gyrolines in the PV gyrovector plane (projection in the $x z$-plane of left cosets); (c) right cosets $\left(\mathbb{R}^{3} / D_{e_{3}}, \sim_{r}\right)=\left\{D_{e_{3}} \oplus u \mathbf{u}, \mathbf{u} \in L_{e_{3}}\right\}$; (d) cogyrolines in the PV gyrovector plane (projection in the $x z$-plane of right cosets).

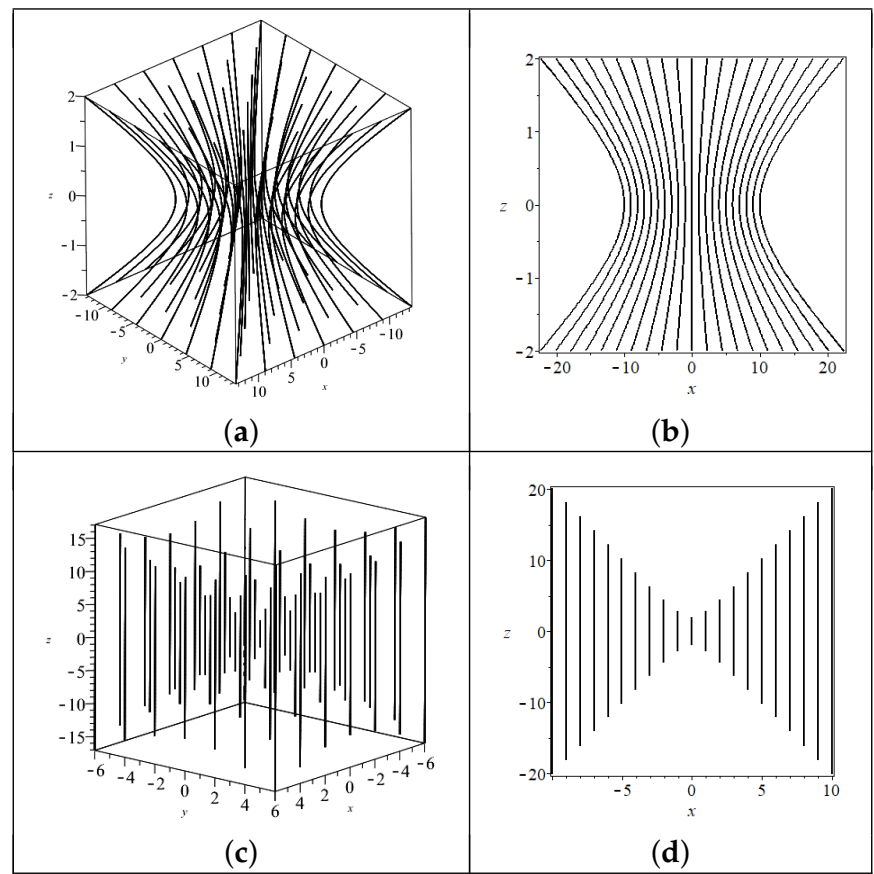

Figure 9. (a) Left cosets $\left(\mathbb{R}^{3} / L_{e_{3}}, \sim_{\ell}\right)=\left\{\mathbf{v} \oplus u L_{e_{3}}, \mathbf{v} \in D_{e_{3}}\right\}$; (b) gyrolines in the PV gyrovector plane (projection in the $x z$-plane of left cosets); (c) right cosets $\left(\mathbb{R}^{3} / L_{e_{3}}, \sim_{r}\right)=\left\{L_{e_{3}} \oplus u \mathbf{v}, \mathbf{v} \in D_{e_{3}}\right\}$; (d) cogyrolines in the PV gyrovector plane (projection in the $x z$-plane of right cosets). 


\subsection{The $S L(2, \mathbb{C})$ General Addition and Chen's Gyrogroup}

Einstein, Möbius, and PV gyrogroups are three different realizations of hyperbolic geometry associated to the Lorentz group. In [13] it was shown that the algebra of the group $\operatorname{SL}(2, \mathbb{C})$ naturally leads to the notion of gyrogroups and gyrovector spaces for dealing with the Lorentz group and its hyperbolic geometry. A general addition for real inner product gyrogroups extended from the group $\operatorname{SL}(2, \mathbb{C})$ is given in the next definition.

Definition $7(\mathrm{SL}(2, \mathbb{C})$ general addition, $[13])$. Let $V=(V,+, \cdot)$ be a real inner product space, and let

$$
\phi: V \rightarrow \mathbb{R}^{+}, \quad \phi: \mathbf{v} \mapsto \phi_{\mathbf{v}}
$$

be a continuous map of a subset $V_{t} \subseteq V$ into the positive real ray $\mathbb{R}^{+}$, taking $\mathbf{v} \in V_{t}$ into $\phi(\mathbf{v})=\phi_{\mathbf{v}} \in \mathbb{R}^{+}$, normalized by the condition $\phi(\mathbf{0})=0$. We assume that $\mathbf{0} \in V_{t}$, and that $V_{t}$ is the identity-connected component of the preimage $\left\{\mathbf{v} \in V: \phi(\mathbf{v}) \in \mathbb{R}^{+}\right\}$of $\mathbb{R}^{+}$under $\phi$. Furthermore, let $f: \mathbb{R}^{+} \rightarrow \mathbb{R}^{+}$be a continuous, bijective self-map of $\mathbb{R}^{+}$, satisfying the condition

$$
f\left(\phi_{\mathbf{v}}\right)=\frac{\|\mathbf{v}\|}{t}
$$

for an arbitrarily fixed positive constant $t$, such that

$$
\frac{\sinh \phi_{\mathbf{v}}}{f\left(\phi_{\mathbf{v}}\right)} \neq 0
$$

for all $\mathbf{v} \in V_{t}$. Then the $S L(2, \mathbb{C})$ general binary operation $\oplus$ in $V_{t}$ is given by the equation

$$
\mathbf{u} \oplus \mathbf{v}=\frac{1}{\frac{\sinh \left(\phi_{\mathbf{u} \oplus \mathbf{v}}\right)}{f\left(\phi_{\mathbf{u} \oplus \mathbf{v}}\right)}}\left(C_{\mathbf{u}, \mathbf{v}} \mathbf{u}+C_{\mathbf{v}} \mathbf{v}\right)
$$

for all $\mathbf{u}, \mathbf{v} \in V_{t}$, where

$$
\begin{aligned}
C_{\mathbf{u}, \mathbf{v}} & =\frac{\sinh \phi_{\mathbf{u}}}{f\left(\phi_{\mathbf{u}}\right)} \cosh \phi_{\mathbf{v}}+\left(\frac{\sinh \phi_{\mathbf{u}}}{f\left(\phi_{\mathbf{u}}\right)}\right)^{2} \frac{\sinh \phi_{\mathbf{v}}}{f\left(\phi_{\mathbf{v}}\right)} \frac{1}{1+\cosh \phi_{\mathbf{u}}} \frac{\mathbf{u} \cdot \mathbf{v}}{t^{2}} \\
C_{\mathbf{v}} & =\frac{\sinh \phi_{\mathbf{v}}}{f\left(\phi_{\mathbf{v}}\right)}
\end{aligned}
$$

and

$$
\cosh \left(\phi_{\mathbf{u} \oplus \mathbf{v}}\right)=\cosh \phi_{\mathbf{u}} \cosh \phi_{\mathbf{v}}+\frac{\sinh \phi_{\mathbf{u}}}{f\left(\phi_{\mathbf{u}}\right)} \frac{\sinh \phi_{\mathbf{v}}}{f\left(\phi_{\mathbf{v}}\right)} \frac{\mathbf{u} \cdot \mathbf{v}}{t^{2}} .
$$

Furthermore, the scalar multiplication in $V_{t}$ is given by the equation

$$
r \otimes \mathbf{v}=t f\left(r f^{-1}\left(\frac{\|\mathbf{v}\|}{t}\right)\right) \frac{\mathbf{v}}{\|\mathbf{v}\|}, \quad \mathbf{v} \neq \mathbf{0}
$$

and $r \otimes \mathbf{0}=\mathbf{0}$, for all $\mathbf{v} \in V_{t}$ and $r \in \mathbb{R}$.

The groupoid $\left(V_{t}, \oplus\right)$ is a gyrocommutative gyrogroup. Moreover, it admits scalar multiplication, $\otimes$, turning itself into a gyrovector space $\left(V_{t}, \oplus, \otimes\right)$. Specifying the function $f$ in various ways results in various binary operations in various subsets $V_{t}$ of $V$. For instance, $t$-Einstein addition is recovered from the $\operatorname{SL}(2, \mathbb{C})$ addition by choosing the function $f(r)=\tanh r, r \in \mathbb{R}^{+}$, the $t$-Möbius addition is recovered from the choice $f(r)=\tanh \left(\frac{r}{2}\right)$, and the $t$-PV addition is recovered from the choice $f(r)=\sinh r$ (see [13] for a detailed discussion). 
In view of (79), we define

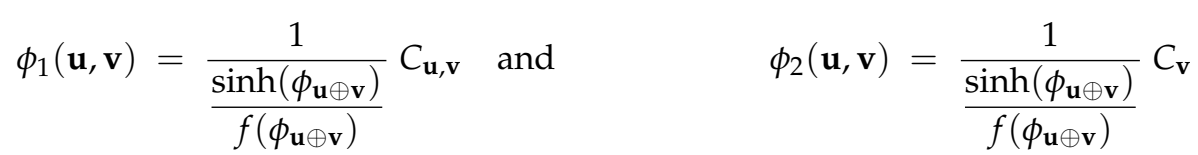

for all $\mathbf{u}, \mathbf{v} \in V_{t}$. It is immediate to see that $\phi_{1}(\mathbf{u}, \mathbf{0})=1$ and $\phi_{2}(\mathbf{0}, \mathbf{v})=1$ for all $\mathbf{u}, \mathbf{v} \in V_{t}$. Let us now see the conditions that $\phi_{1}$ and $\phi_{2}$ have to satisfy such that hypothesis (H1) and (H2) hold. By construction, hypothesis (H1) naturally holds. For the hypothesis (H2), consider $\mathbf{u}, \mathbf{v} \in V_{t}$, with $\mathbf{u}+\mathbf{v} \in V_{t}$ and $\mathbf{u} \cdot \mathbf{v}=0$. Since $\lambda_{1} \mathbf{u} \perp \lambda_{2} \mathbf{v}$, for all $\lambda_{1}, \lambda_{2} \in \mathbb{R}$ it follows from (80) that the system of equations

$$
\phi_{1}\left(\lambda_{1} \mathbf{u}, \lambda_{2} \mathbf{v}\right) \lambda_{1} \mathbf{u}=\mathbf{u} \text { and } \phi_{2}\left(\lambda_{1} \mathbf{u}, \lambda_{2} \mathbf{v}\right) \lambda_{2} \mathbf{v}=\mathbf{v}
$$

is equivalent to

$$
\frac{f\left(\phi_{\lambda_{1} \mathbf{u} \oplus \lambda_{2} \mathbf{v}}\right)}{\sinh \left(\phi_{\lambda_{1} \mathbf{u} \oplus \lambda_{2} \mathbf{v}}\right)} \frac{\sinh \left(\phi_{\mathbf{1}_{\mathbf{1}} \mathbf{u}}\right)}{f\left(\phi_{\mathbf{v} \mathbf{u}}\right)} \cosh \left(\phi_{\lambda_{2} \mathbf{v}}\right) \lambda_{1}=1 \quad \text { and } \quad \frac{f\left(\phi_{\lambda_{1} \mathbf{u} \oplus \lambda_{2} \mathbf{v}}\right)}{\sinh \left(\phi_{\lambda_{1} \mathbf{u} \oplus \lambda_{2} \mathbf{v}}\right)} \frac{\sinh \left(\phi_{\lambda_{2} \mathbf{v}}\right)}{f\left(\phi_{\lambda_{2} \mathbf{v}}\right)} \lambda_{2}=1 .
$$

The solution of the previous system depends on the choice of the functions $f$ and $\phi$. In the cases of Einstein, Möbius, and PV gyrogroups we already proved that the system has a unique solution. In [1] it was obtained another example of a real inner product gyrogroup considering the function $f(r)=\sinh \frac{r}{2}, r \in \mathbb{R}^{+}$in the general $\operatorname{SL}(2, \mathbb{C})$ addition (79). This addition was called Chen's addition and is given by

$$
\mathbf{u} \oplus_{C} \mathbf{v}=\frac{\left(\frac{2}{\beta_{\mathbf{v}}}-\beta_{\mathbf{v}}+2 \beta_{\mathbf{u}} \frac{\mathbf{u} \cdot \mathbf{v}}{t^{2}}\right) \mathbf{u}+\beta_{\mathbf{u} \mathbf{v}}}{\sqrt{\beta_{\mathbf{u}}^{2} \beta_{\mathbf{v}}^{2}-\left(\beta_{\mathbf{u}}^{2}+\beta_{\mathbf{v}}^{2}\right)+2\left(1+\beta_{\mathbf{u}} \beta_{\mathbf{v}} \frac{\mathbf{u} \cdot \mathbf{v}}{t^{2}}\right)}}
$$

for all $\mathbf{u}, \mathbf{v} \in V_{t}=V$, where $\beta_{\mathbf{v}}=\frac{1}{\sqrt{1+\frac{\|\mathbf{v}\|^{2}}{t^{2}}}}, \mathbf{v} \in V$. Chen's addition satisfies the following beta identity:

$$
\frac{1}{\beta_{\mathbf{u} \oplus_{C} \mathbf{v}}}=\frac{1}{\beta_{\mathbf{u}}} \frac{1}{\beta_{\mathbf{v}}} \sqrt{\beta_{\mathbf{u}}^{2} \beta_{\mathbf{v}}^{2}-\left(\beta_{\mathbf{u}}^{2}+\beta_{\mathbf{v}}^{2}\right)+2\left(1+\beta_{\mathbf{u}} \beta_{\mathbf{v}} \frac{\mathbf{u} \cdot \mathbf{v}}{t^{2}}\right)} \text {. }
$$

The scalar multiplication associated with Chen's addition is given by

$$
r \otimes_{C} \mathbf{v}=t \sinh \left(r \sinh ^{-1} \frac{\|\mathbf{v}\|}{t}\right) \frac{\mathbf{v}}{\|\mathbf{v}\|}
$$

for $\mathbf{v} \neq \mathbf{0}$ and $r \otimes_{C} \mathbf{0}=\mathbf{0}$. Thus, $\left(V, \oplus_{C}, \otimes_{C}\right)$ becomes a gyrovector space. The gyrogroup identity of $\left(V, \oplus_{C}\right)$ is the zero vector $\mathbf{0}$. Further, $\ominus_{C} \mathbf{u}=-\mathbf{u}$ for all $\mathbf{u} \in V$. In view of (81), we define

$$
\begin{aligned}
& \phi_{1}(\mathbf{u}, \mathbf{v})=\frac{\frac{2}{\beta_{\mathbf{v}}}-\beta_{\mathbf{v}}+2 \beta_{\mathbf{u}} \frac{\mathbf{u} \cdot \mathbf{v}}{t^{2}}}{\sqrt{\beta_{\mathbf{u}}^{2} \beta_{\mathbf{v}}^{2}-\left(\beta_{\mathbf{u}}^{2}+\beta_{\mathbf{v}}^{2}\right)+2\left(1+\beta_{\mathbf{u}} \beta_{\mathbf{v}} \frac{\mathbf{u} \cdot \mathbf{v}}{t^{2}}\right)}} \\
& \phi_{2}(\mathbf{u}, \mathbf{v})=\frac{\beta_{\mathbf{u}}}{\sqrt{\beta_{\mathbf{u}}^{2} \beta_{\mathbf{v}}^{2}-\left(\beta_{\mathbf{u}}^{2}+\beta_{\mathbf{v}}^{2}\right)+2\left(1+\beta_{\mathbf{u}} \beta_{\mathbf{v}} \frac{\mathbf{u} \cdot \mathbf{v}}{t^{2}}\right)}}
\end{aligned}
$$


for all $\mathbf{u}, \mathbf{v} \in V$. It is clear that Chen's gyrogroup satisfies (H1) by construction. Now, we suppose that $\mathbf{u}, \mathbf{v} \in V, \mathbf{u}+\mathbf{v} \in V$, and $\mathbf{u} \cdot \mathbf{v}=0$. Using computer algebra we can find that the unique solution of the system of equations

$$
\left\{\begin{array}{l}
\phi_{1}\left(\lambda_{1} \mathbf{u}, \lambda_{2} \mathbf{v}\right) \lambda_{1} \mathbf{u}=\mathbf{u} \\
\phi_{2}\left(\lambda_{1} \mathbf{u}, \lambda_{2} \mathbf{v}\right) \lambda_{2} \mathbf{v}=\mathbf{v}
\end{array}\right.
$$

is given by

$$
\begin{aligned}
& \lambda_{1}=\frac{1}{\frac{\sqrt{2}\|\mathbf{u}\|}{t}} \sqrt{\frac{1+\frac{2}{t^{2}}\left(\|\mathbf{u}\|^{2}+\|\mathbf{v}\|^{2}\right)}{\sqrt{1+4\left(1+\frac{\|\mathbf{u}\|^{2}}{t^{2}}\right) \frac{\|\mathbf{v}\|^{2}}{t^{2}}+4 \frac{\|\mathbf{v}\|^{4}}{t^{4}}}}-1}, \\
& \lambda_{2}=\frac{1}{\frac{\sqrt{2}\|\mathbf{v}\|}{t}} \sqrt{\sqrt{1+4\left(1+\frac{\|\mathbf{u}\|^{2}}{t^{2}}\right) \frac{\|\mathbf{v}\|^{2}}{t^{2}}+4 \frac{\|\mathbf{v}\|^{4}}{t^{4}}}-1 .}
\end{aligned}
$$

Now, let $P$ be a linear subspace of $V$. The next three theorems are consequences of Theorems 5,8 , 10, 13 obtained in Section 3.

Theorem 31. Let $P$ be a subgyrogroup of $\left(\mathbb{R}^{n}, \oplus_{C}\right)$. For all $\mathbf{w} \in \mathbb{R}^{n}$ such that $\mathbf{w}=\mathbf{u}+\mathbf{v}$, with $\mathbf{u} \in P$ and $\mathbf{v} \in P^{\perp}$, the unique orthogonal decompositions of $\mathbf{w}$ according to $\oplus_{C}$ are given by

$$
\mathbf{w}=\lambda_{1}(\mathbf{u}, \mathbf{v}) \mathbf{u} \oplus_{C} \lambda_{2}(\mathbf{u}, \mathbf{v}) \mathbf{v} \text { and } \mathbf{w}=\lambda_{3}(\mathbf{u}, \mathbf{v}) \mathbf{v} \oplus_{C} \lambda_{4}(\mathbf{u}, \mathbf{v}) \mathbf{u},
$$

where

$$
\begin{aligned}
& \lambda_{1}(\mathbf{u}, \mathbf{v})=\frac{1}{\frac{\sqrt{2}\|\mathbf{u}\|}{t}} \sqrt{\frac{1+\frac{2}{t^{2}}\left(\|\mathbf{u}\|^{2}+\|\mathbf{v}\|^{2}\right)}{\sqrt{1+\frac{4}{\beta_{\mathbf{u}}^{2}} \frac{\|\mathbf{v}\|^{2}}{t^{2}}+4 \frac{\|\mathbf{v}\|^{4}}{t^{4}}}}-1} \\
& \lambda_{2}(\mathbf{u}, \mathbf{v})=\frac{1}{\frac{\sqrt{2}\|\mathbf{v}\|}{t}} \sqrt{\sqrt{1+\frac{4}{\beta_{\mathbf{u}}^{2}} \frac{\|\mathbf{v}\|^{2}}{t^{2}}+4 \frac{\|\mathbf{v}\|^{4}}{t^{4}}}-1} \\
& \lambda_{3}(\mathbf{u}, \mathbf{v})=\frac{1}{\frac{\sqrt{2}\|\mathbf{v}\|}{t}} \sqrt{\frac{1+\frac{2}{t^{2}}\left(\|\mathbf{u}\|^{2}+\|\mathbf{v}\|^{2}\right)}{\sqrt{1+\frac{4}{\beta_{\mathbf{v}}^{2}} \frac{\|\mathbf{u}\|^{2}}{t^{2}}+4 \frac{\|\mathbf{u}\|^{4}}{t^{4}}}}-1} \\
& \lambda_{4}(\mathbf{u}, \mathbf{v})=\frac{1}{\frac{\sqrt{2}\|\mathbf{u}\|}{t}} \sqrt{\sqrt{1+\frac{4}{\beta_{\mathbf{v}}^{2}} \frac{\|\mathbf{u}\|^{2}}{t^{2}}+4 \frac{\|\mathbf{u}\|^{4}}{t^{4}}}-1}
\end{aligned}
$$

For each $\mathbf{w} \in V$, if $\mathbf{w}=\mathbf{u}+\mathbf{v}$, with $\mathbf{u} \in P$ and $\mathbf{v} \in P^{\perp}$ is the unique decomposition of $\mathbf{w}$, according to the orthogonal direct sum decomposition $V=P \oplus P^{\perp}$, then the orthogonal gyroprojections of Chen's gyrogroup $\left(V, \oplus_{C}\right)$ onto $P$ and $P^{\perp}$ are given by 


$$
\begin{aligned}
& \mathcal{P}_{C}^{\ell}: V=P \oplus_{C} P^{\perp} \rightarrow P, \quad \mathcal{P}_{C}^{\ell}(\mathbf{w})=\sqrt{\frac{1+\frac{2}{t^{2}}\left(\|\mathbf{u}\|^{2}+\|\mathbf{v}\|^{2}\right)}{\sqrt{1+\frac{4}{\beta_{\mathbf{u}}^{2}} \frac{\|\mathbf{v}\|^{2}}{t^{2}}+4 \frac{\|\mathbf{v}\|^{4}}{t^{4}}}}-1} \frac{1}{\frac{\sqrt{2}\|\mathbf{u}\|}{t}} \mathbf{u}, \\
& \mathcal{Q}_{C}^{r}: V=P \oplus_{C} P^{\perp} \rightarrow P^{\perp}, \quad \mathcal{Q}_{C}^{r}(\mathbf{w})=\sqrt{\sqrt{1+\frac{4}{\beta_{\mathbf{u}}^{2}} \frac{\|\mathbf{v}\|^{2}}{t^{2}}+4 \frac{\|\mathbf{v}\|^{4}}{t^{4}}}-1 \frac{1}{\frac{\sqrt{2}\|\mathbf{v}\|}{t}} \mathbf{v},} \\
& \mathcal{Q}_{C}^{\ell}: V=P^{\perp} \oplus_{C} P \rightarrow P^{\perp}, \quad \mathcal{Q}_{C}^{\ell}(\mathbf{w})=\sqrt{\frac{1+\frac{2}{t^{2}}\left(\|\mathbf{u}\|^{2}+\|\mathbf{v}\|^{2}\right)}{\sqrt{1+\frac{4}{\beta_{\mathbf{v}}^{2}} \frac{\|\mathbf{u}\|^{2}}{t^{2}}+4 \frac{\|\mathbf{u}\|^{4}}{t^{4}}}}-1 \frac{1}{\frac{\sqrt{2}\|\mathbf{v}\|}{t}} \mathbf{v},} \\
& \mathcal{P}_{C}^{r}: V=P^{\perp} \oplus_{C} P \rightarrow P, \quad \mathcal{P}_{C}^{r}(\mathbf{w})=\sqrt{\sqrt{1+\frac{4}{\beta_{\mathbf{v}}^{2}} \frac{\|\mathbf{u}\|^{2}}{t^{2}}+4 \frac{\|\mathbf{u}\|^{4}}{t^{4}}}-1} \frac{1}{\frac{\sqrt{2}\|\mathbf{u}\|}{t}} \mathbf{u} .
\end{aligned}
$$

Theorem 32. Let $P$ be a linear subspace of $V$. Then the sets $\left\{b \oplus_{C} P: b \in P^{\perp}\right\}$ and $\left\{P \oplus_{C} b: b \in P^{\perp}\right\}$ are disjoint partitions of $V$, that is,

$$
V=\bigcup_{b \in P^{\perp}}\left(b \oplus_{C} P\right) \text { and } V=\bigcup_{b \in P^{\perp}}\left(P \oplus_{C} b\right) .
$$

Moreover, $\left(V / P, \sim_{\ell}\right)=\left\{b \oplus_{C} P: b \in P^{\perp}\right\}$ and $\left(V / P, \sim_{r}\right)=\left\{P \oplus_{C} b: b \in P^{\perp}\right\}$.

Theorem 33. Let $P$ be a linear subspace of $V$. Then the following are gyrogroup isomorphisms:

$$
\left(V / P, \sim_{\ell}\right) \cong\left(P^{\perp}, \oplus_{C}\right) \cong\left(V / P, \sim_{r}\right)
$$

Finally, when $t \rightarrow+\infty, \oplus_{C}$ reduces to ordinary vector addition of $V$. Surprisingly, $\mathcal{P}_{C}^{\ell}$ and $\mathcal{P}_{C}^{r}$ reduce again to the ordinary projection $\mathcal{P}(\mathbf{u}+\mathbf{v})=\mathbf{u}$, and $\mathcal{Q}_{C}^{\ell}$ and $\mathcal{Q}_{C}^{r}$ reduce to the ordinary projection $\mathcal{Q}(\mathbf{u}+\mathbf{v})=\mathbf{v}$. Further, the equivalence relation (29) reflects the left coset relation in $(V,+)$ :

$$
\mathbf{u} \sim_{\ell} \mathbf{v} \quad \text { if and only if } \quad-\mathbf{v}+\mathbf{u} \in P
$$

and the equivalence relation (31) reflects the right coset relation in $(V,+)$ :

$$
\mathbf{u} \sim_{r} \mathbf{v} \quad \text { if and only if } \quad \mathbf{u}-\mathbf{v} \in P .
$$

Let us now find $\mu_{1}(\mathbf{u}, \mathbf{v})$ and $\mu_{2}(\mathbf{u}, \mathbf{v})$ for Chen's gyrogroup, according to Theorem 6.

Theorem 34. Let $\mathbf{u}, \mathbf{v} \in\left(V, \oplus_{C}\right)$ such that $\mathbf{u} \cdot \mathbf{v}=0$. Then

$$
\mathbf{u} \oplus_{C} \mathbf{v}=\mu_{1}(\mathbf{u}, \mathbf{v}) \mathbf{v} \oplus_{C} \mu_{2}(\mathbf{u}, \mathbf{v}) \mathbf{u},
$$

where

$$
\begin{aligned}
& \mu_{1}(\mathbf{u}, \mathbf{v})=\frac{1}{\frac{\sqrt{2}\|\mathbf{v}\|}{t}} \sqrt{\frac{1+\frac{2}{t^{2}}\left(\|\mathbf{u}\|^{2}+\|\mathbf{v}\|^{2}+2 \frac{\|\mathbf{u}\|^{2}\|\mathbf{v}\|^{2}}{t^{2}}\right)}{\sqrt{1+\frac{4}{\beta_{\mathbf{u}}^{2}} \frac{\|\mathbf{u}\|^{2}}{t^{2}}\left(1+\frac{2\|\mathbf{v}\|^{2}}{t^{2}}\right)^{2}}}-1}, \\
& \mu_{2}(\mathbf{u}, \mathbf{v})=\frac{1}{\frac{\sqrt{2}\|\mathbf{u}\|}{t}} \sqrt{\sqrt{1+\frac{4}{\beta_{\mathbf{u}}^{2}} \frac{\|\mathbf{u}\|^{2}}{t^{2}}\left(1+\frac{2\|\mathbf{v}\|^{2}}{t^{2}}\right)}-1} .
\end{aligned}
$$

The proof follows by straightforward computations using (27), (82), and (84). 
In Figure 10 we show the plots of $\mu_{1}(\mathbf{u}, \mathbf{v})\|\mathbf{v}\|$ and $\mu_{2}(\mathbf{v}, \mathbf{u})\|\mathbf{v}\|$ for Chen's gyrogroup.

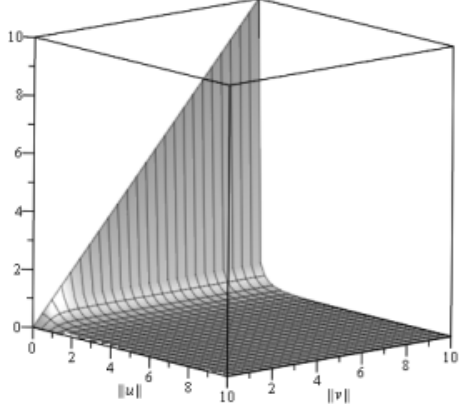

(a)

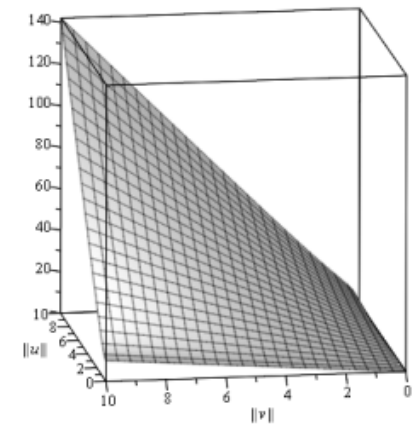

(b)

Figure 10. (a) Plot of $\mu_{1}(\mathbf{u}, \mathbf{v})\|\mathbf{v}\|$ with $t=1$ and $\|u\|,\|v\| \in[0,10]$; (b) plot of $\mu_{2}(\mathbf{v}, \mathbf{u})\|\mathbf{v}\|$ with $t=1$ and $\|u\|,\|v\| \in[0,10]$, for Chen's gyrogroup.

Since $\lim _{\|\mathbf{v}\| \rightarrow+\infty} \mu_{1}(\mathbf{u}, \mathbf{v})\|\mathbf{v}\|=\frac{t}{2} \sqrt{\frac{t^{2}+2\|\mathbf{u}\|^{2}}{\|\mathbf{u}\| \sqrt{t^{2}+\|\mathbf{u}\|^{2}}}-2}$ then for all $\mathbf{u} \in P \backslash\{\mathbf{0}\}$ we have that $\mu_{1}(\mathbf{u}, \mathbf{v})\|\mathbf{v}\| \in\left[0, \frac{t}{2} \sqrt{\frac{t^{2}+2\|\mathbf{u}\|^{2}}{\|\mathbf{u}\| \sqrt{t^{2}+\|\mathbf{u}\|^{2}}}-2}\left[\subsetneq\left[0,+\infty\left[\right.\right.\right.\right.$, for all $\mathbf{v} \in P^{\perp}$. This implies that $\tau_{\mathbf{u}}^{(2)}$ is only a local section for the fiber bundles $\pi_{1}$ and $\pi_{3}$ defined in (38). In the case when $\mathbf{u}=\mathbf{0}$ the section $\tau_{\mathbf{0}}^{(2)}$ is a global section as seen in (41).

Regarding $\mu_{2}(\mathbf{v}, \mathbf{u})\|\mathbf{v}\|$ we have that for each $\mathbf{u} \in P$ it is a strictly increasing function in the variable $\|\mathbf{v}\|$ such that $\mu_{2}(\mathbf{0}, \mathbf{u})\|\mathbf{0}\|=0$ and $\lim _{\|\mathbf{v}\| \rightarrow+\infty} \mu_{2}(\mathbf{v}, \mathbf{u})\|\mathbf{v}\|=+\infty$. Therefore, we conclude that $\left\{\mu_{2}(\mathbf{v}, \mathbf{u}) \mathbf{v}: \mathbf{v} \in P^{\perp}\right\}=P^{\perp}$. Hence, for any $\mathbf{u} \in P$, it follows that $\tau_{\mathbf{u}}^{(4)}$ is a global section for the fiber bundles $\pi_{2}$ and $\pi_{4}$ defined in (38).

In the case of Chen's gyrogroup we have the same duality relations as in Theorem 30 . To visualize the left and right cosets in this case we consider $V$ the 3-dimensional space $\mathbb{R}^{3}$ and we show in Figures 11 and 12 the cosets obtained from the orthogonal decompositions of the gyrogroup $\left(\mathbb{R}^{3}, \oplus_{C}\right)$ by the subgyrogroups $L_{e_{3}}$ and $D_{e_{3}}$.

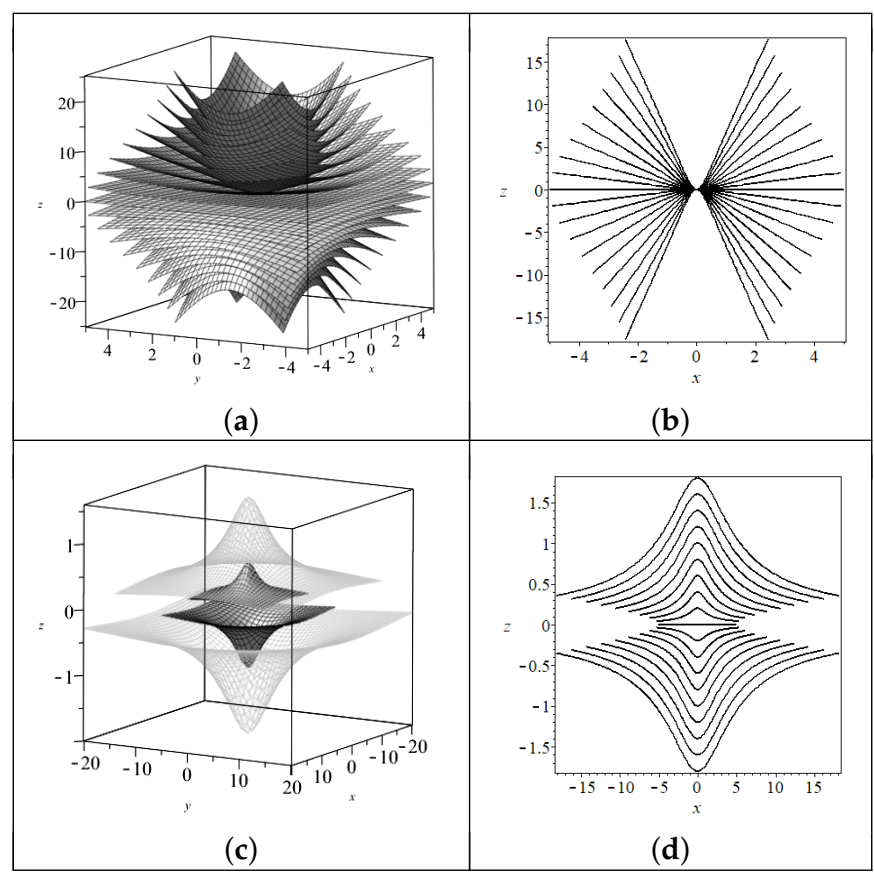

Figure 11. (a) Left cosets $\left(\mathbb{R}^{3} / D_{e_{3}}, \sim_{\ell}\right)=\left\{\mathbf{u} \oplus_{C} D_{e_{3}}, \mathbf{u} \in L_{e_{3}}\right\} ;$ (b) gyrolines in the Chen's gyrovector plane (projection in the $x z$-plane of left cosets); (c) right cosets $\left(\mathbb{R}^{3} / D_{e_{3}}, \sim_{r}\right)=\left\{D_{e_{3}} \oplus_{C} \mathbf{u}, \mathbf{u} \in L_{e_{3}}\right\}$; (d) cogyrolines in the Chen's gyrovector plane (projection in the $x z$-plane of right cosets). 


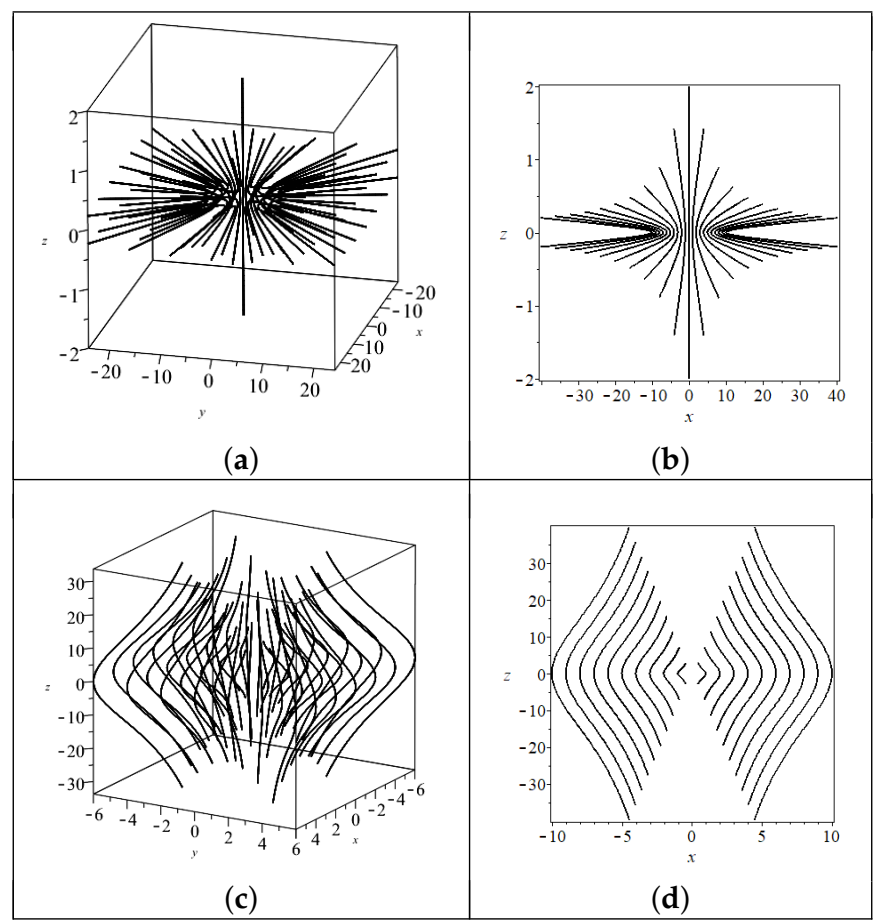

Figure 12. (a) Left cosets $\left(\mathbb{R}^{3} / L_{e_{3}}, \sim_{\ell}\right)=\left\{\mathbf{v} \oplus_{C} L_{e_{3}}, \mathbf{v} \in D_{e_{3}}\right\} ;(\mathbf{b})$ gyrolines in the Chen's gyrovector plane (projection in the $x z$-plane of left cosets); (c) right cosets $\left(\mathbb{R}^{3} / L_{e_{3}}, \sim_{r}\right)=\left\{L_{e_{3}} \oplus_{C} \mathbf{v}, \mathbf{v} \in D_{e_{3}}\right\}$; (d) cogyrolines in the Chen's gyrovector plane (projection in the $x z$-plane of right cosets).

\section{Conclusions}

We generalized the study of factorization of Möbius gyrogroups to that of real inner product gyrogroups and proved the Unique Decomposition Theorem. This is the main theorem that leads to other remarkable results proved in this work. It resembles the standard theorem in linear algebra that every inner product space has an orthogonal direct sum decomposition associated to its finite-dimensional subspace. Because of the nonassociativity and the noncommutativity, we defined suitable equivalence relations on real inner product gyrogroups. With the equivalence relations we could partition a real inner product gyrogroup into left and right coset spaces. The four gyrogroups studied to confirm the general theory allow explicit calculations of the gyroprojectors and the left and right cosets. There are several possible applications of our results. We mention, for example, the construction of orthogonal gyroexpansions with respect to an orthogonal basis in a real inner product gyrogroup, or the construction of integral transforms such as the wavelet transform on some manifolds, such as the sphere, the ball, or the hyperboloid (cf. $[6,14])$. Finally, it would be interesting to generalize these results to complex gyrogroups and to the novel bi-gyrogroups (see [15]), that give a parametrization of generalized Lorentz groups $\mathrm{SO}(m, n), m, n \in \mathbb{N}$, in pseudo-Euclidean spaces of signature $(m, n)$.

Author Contributions: All authors contributed equally to this article. All authors have read and agreed to the published version of the manuscript.

Funding: The work of M. Ferreira was supported by the Center for Research and Development in Mathematics and Applications (CIDMA) through the Portuguese Foundation for Science and Technology ("FCT - Fundação para a Ciência e a Tecnologia"), within project UIDB/04106/2020. The work of T. Suksumran was supported by the Research Center in Mathematics and Applied Mathematics, Chiang Mai University.

Acknowledgments: T. Suksumran wishes to thank the hospitality of the Mathematics Department at the University of Aveiro, Portugal, where part of this work has been made during a research visit.

Conflicts of Interest: The authors declare no conflict of interest. 


\section{Abbreviations}

The following abbreviations are used in this manuscript:

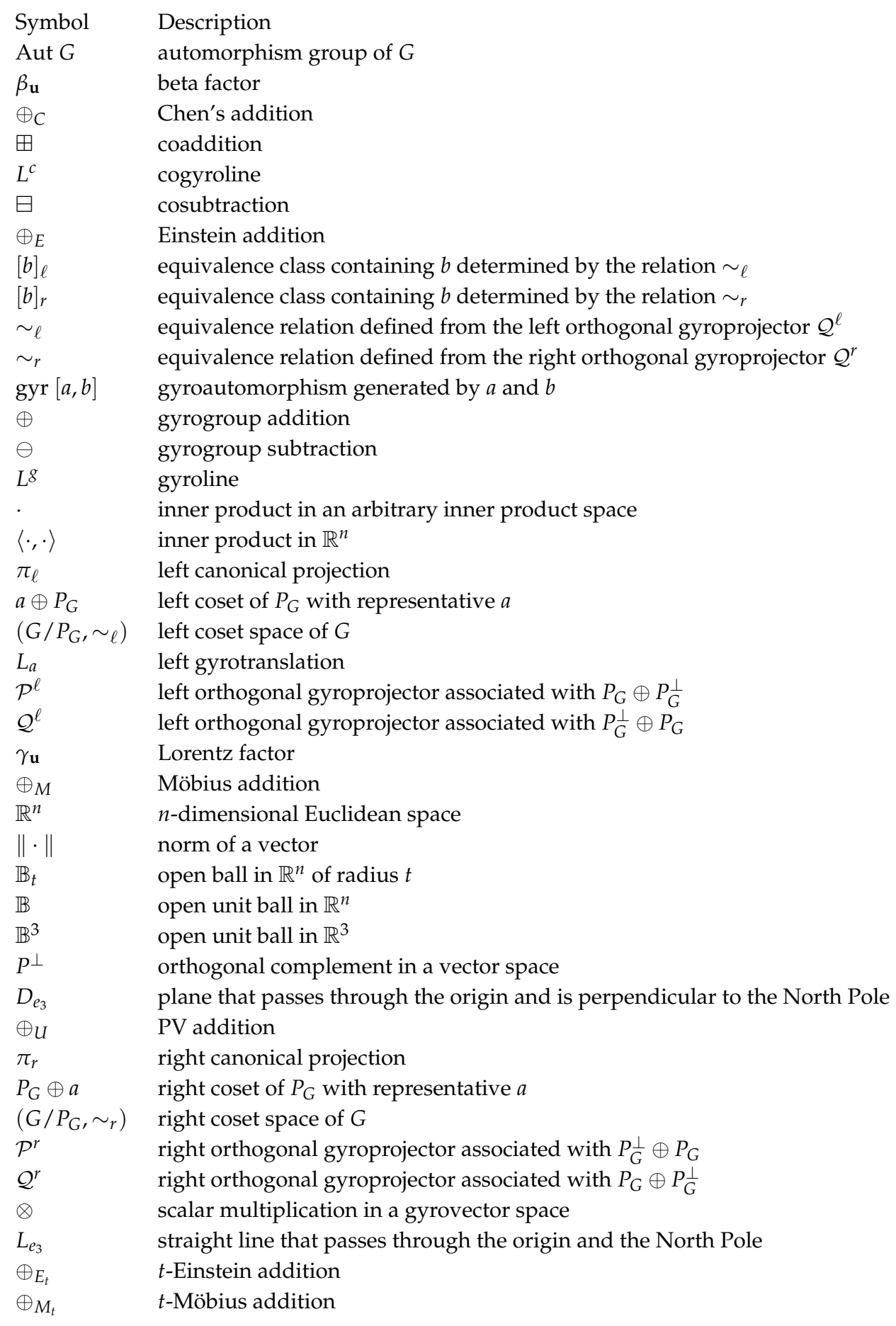

\section{References}

1. Ungar, A.A. Thomas rotation and the parametrization of the Lorentz transformation group. Found. Phys. Lett. 1988, 1, 57-89. [CrossRef]

2. Ungar, A.A. Analytic Hyperbolic Geometry: Mathematical Foundations and Applications; World Scientific: Hackensack, NJ, USA, 2005.

3. Ungar, A.A. Analytic Hyperbolic Geometry and Albert Einstein's Special Theory of Relativity; World Scientific: Hackensack, NJ, USA, 2008. 
4. Ferreira, M. Factorizations of Möbius gyrogroups. Adv. Appl. Clifford Algebr. 2009, 19, 303-323. [CrossRef]

5. Ferreira, M.; Ren, G. Möbius gyrogroups: A Clifford algebra approach. J. Algebra 2011, 328, $230-253$. [CrossRef]

6. Ferreira, M. Spherical continuous wavelet transforms arising from sections of the Lorentz group. Appl. Comput. Harmon. Anal. 2009, 26, 212-229. [CrossRef]

7. Abe, T.; Watanabe, K. Finitely generated gyrovector subspaces and orthogonal gyrodecomposition in the Möbius gyrovector space. J. Math. Anal. Appl. 2017, 449, 77-90. [CrossRef]

8. Suksumran, T.; Wiboonton, K. Isomorphism theorems for gyrogroups and L-subgyrogroups. J. Geom. Symmetry Phys. 2015, 37, 67-83.

9. Suksumran, T. The Algebra of Gyrogroups: Cayley's Theorem, Lagrange's Theorem, and Isomorphism Theorems. In Essays in Mathematics and its Applications, In Honor of Vladimir Arnold; Rassias, T.M., Pardalos, P.M., Eds.; Springer: Cham, Switzerland, 2016; pp. 369-437.

10. Ungar, A.A. Einstein's velocity addition law and its hyperbolic geometry. Comput. Math. Appl. 2007, 53, 1228-1250.

11. Ungar, A.A. From Möbius to gyrogroups. Am. Math. Mon. 2008, 115, 138-144. [CrossRef]

12. Ungar, A.A. Extension of the unit disk gyrogroup into the unit ball of any real inner product space. J. Math. Anal. Appl. 1996, 202,1040-1057. [CrossRef]

13. Chen, J.; Ungar, A.A. From the group SL $(2, \mathrm{C})$ to gyrogroups and gyrovector spaces and hyperbolic geometry. Found. Phys. 2001, 31, 1611-1639. [CrossRef]

14. Martinez-Moreno, J.; Watanabe, K. Orthogonal Gyroexpansion in Möbius Gyrovector Spaces. J. Funct. Spaces 2017, 2017, 1518254.

15. Teerapong, S.; Ungar, A.A. Bi-Gyrogroup: The Group-Like Structure Induced by Bi-Decomposition of Groups. Math. Interdisc. Res. 2016, 1, 111-142.

(C) 2020 by the authors. Licensee MDPI, Basel, Switzerland. This article is an open access article distributed under the terms and conditions of the Creative Commons Attribution (CC BY) license (http:/ / creativecommons.org/licenses/by/4.0/). 